\title{
REVIEW ARTICLE OPEN Targeted therapies in gynecological cancers: a comprehensive review of clinical evidence
}

\author{
Qiao Wang ${ }^{1}$, Hongling Peng ${ }^{1}$, Xiaorong $\mathrm{Qi}^{1}$, Min $\mathrm{Wu}^{2}$ and Xia Zhao ${ }^{1}$ \\ Advanced and recurrent gynecological cancers are associated with poor prognosis and lack of effective treatment. The \\ developments of the molecular mechanisms on cancer progression provide insight into novel targeted therapies, which are \\ emerging as groundbreaking and promising cancer treatment strategies. In gynecologic malignancies, potential therapeutic \\ targeted agents include antiangiogenic agents, poly (ADP-ribose) polymerase (PARP) inhibitors, tumor-intrinsic signaling pathway \\ inhibitors, selective estrogen receptor downregulators, and immune checkpoint inhibitors. In this article, we provide a \\ comprehensive review of the clinical evidence of targeted agents in gynecological cancers and discuss the future implication.
}

Signal Transduction and Targeted Therapy (2020)5:137

; https://doi.org/10.1038/s41392-020-0199-6

\section{INTRODUCTION}

Gynecological malignancies, mainly including ovarian, cervical, and endometrial cancer, seriously affect the health of women worldwide, contributing considerably to the global cancer burden. Epithelial ovarian cancer (OC) comprises $\sim 90 \%$ of the malignant ovarian neoplasms, which is one of the leading causes of death in women. ${ }^{1,2}$ The 5 -year overall survival (OS) rate of OC is $\sim 47 \%$ for all stages, and $>70 \%$ of patients are diagnosed at the advanced stage with an even lower 5-year OS rate.,4 The standard-of-care fist-line treatments for $\mathrm{OC}$ are debulking surgery and perioperative platinum-based chemotherapy. 5,6 Although the response rate of the first-line treatment is high, most of the patients will eventually experience relapses within the subsequent 3 years. ${ }^{7}$ At first relapse, $\sim 20-25 \%$ of patients have platinum-resistant (disease recurs $\leq 6$ months from the last platinum-based chemotherapy) or platinum-refractory (disease progress during or within 4 weeks of platinum-based chemotherapy) disease, with poor prognosis., ${ }^{8,9}$ In the platinum-resistant disease, single non-platinum agent is used, such as paclitaxel, docetaxel, pegylated liposomal doxorubicin (PLD), gemcitabine and topotecan. However, the response rates and outcomes are disappointing. Cervical cancer (CC), as the fourth most common female cancer globally, is also a major health problem especially for women in developing countries. ${ }^{10}$ High-risk human papilloma virus (HPV) infection is considered to be responsible for more than $90 \%$ of CC development. ${ }^{11} \mathrm{HPV}$ overexpresses E6 and E7 oncoproteins which inhibit TP53 and RB1 proteins from altering cell cycle, apoptosis, and DNA repair. ${ }^{12,13}$ Thus, HPV testing is an important part of CC screening, and immunization against HPV (e.g., vaccines) has been designed to prevent CC. ${ }^{14,15}$ With early screening and effective treatments such as radical surgery or concurrent chemoradiation (a combination of radiation and chemotherapy), the cure rate of CC can reach $80 \%$ in the early-stage disease (FIGO stage I-II). The 5-year OS rate for all stages is $\sim 66 \%$. However, treatment options are limited and the survival rate is low for patients who present with distant metastatic disease, as well as those with unresectable recurrent disease and those who recur at distant. Endometrial cancer (EC), also known as uterine cancer, is the sixth most common female cancer. ${ }^{10,16}$ Elevated estrogen levels and increasing age are wellknown risk factors of EC. ${ }^{17,18}$ Thus, the incidence of EC is increasing due to the increased life expectancy and obesity (causing elevated estrogen level). The standard treatment consists of surgery with or without adjuvant radiotherapy and/or chemotherapy, which is based on the risk of disease recurrence. ${ }^{19}$ Traditionally, EC has been classified in two types mainly according to histology and estrogen dependence. Furthermore, the Cancer Genome Atlas (TCGA) identified EC into four molecular subgroups: polymerase epsilon (POLE) ultramutated, microsatellite instability hypermutated, copy-number low, and copy-number high, each with a distinct prognosis. ${ }^{20}$ Most low-risk patients with early-stage disease can be cured by surgery and have good prognoses. However, the prognosis for advanced EC is poor with 5-year OS rate of $40-65 \%$ in stage III and $15-17 \%$ in stage IV disease, respectively. ${ }^{21}$ All those malignancies, when progressed to the advanced stage, have very poor prognoses under conventional treatment. Due to the lack of effective treatment for advancedstage, refractory, recurrent, and drug-resistance disease, we are facing very tough challenges. However, based on the improved understanding of the mechanisms on cancer progression, targeted therapies are emerging as groundbreaking and promising treatment strategies.

In targeted therapies, individual patients are treated by agents targeting the changes in tumor cells that help them grow, divide, and spread. Currently in gynecological malignancies, potential therapeutic targets include tumor-intrinsic signaling pathways, angiogenesis, homologous-recombination deficiency (HDR),

\footnotetext{
'Department of Gynecology and Obstetrics, Development and Related Diseases of Women and Children Key Laboratory of Sichuan Province, Key Laboratory of Birth Defects and Related Diseases of Women and Children, Ministry of Education, West China Second Hospital, Sichuan University, Chengdu, Sichuan 610041, P.R. China and ${ }^{2}$ Department of Biomedical Sciences, School of Medicine and Health Sciences, University of North Dakota, Grand Forks, ND 58203, USA

Correspondence: Xia Zhao (xia-zhao@126.com)

These authors contributed equally: Qiao Wang, Hongling Peng
}

Received: 3 February 2020 Revised: 12 May 2020 Accepted: 20 May 2020

Published online: 29 July 2020 
Table 1. FDA-approved targeted drugs for gynecological cancers

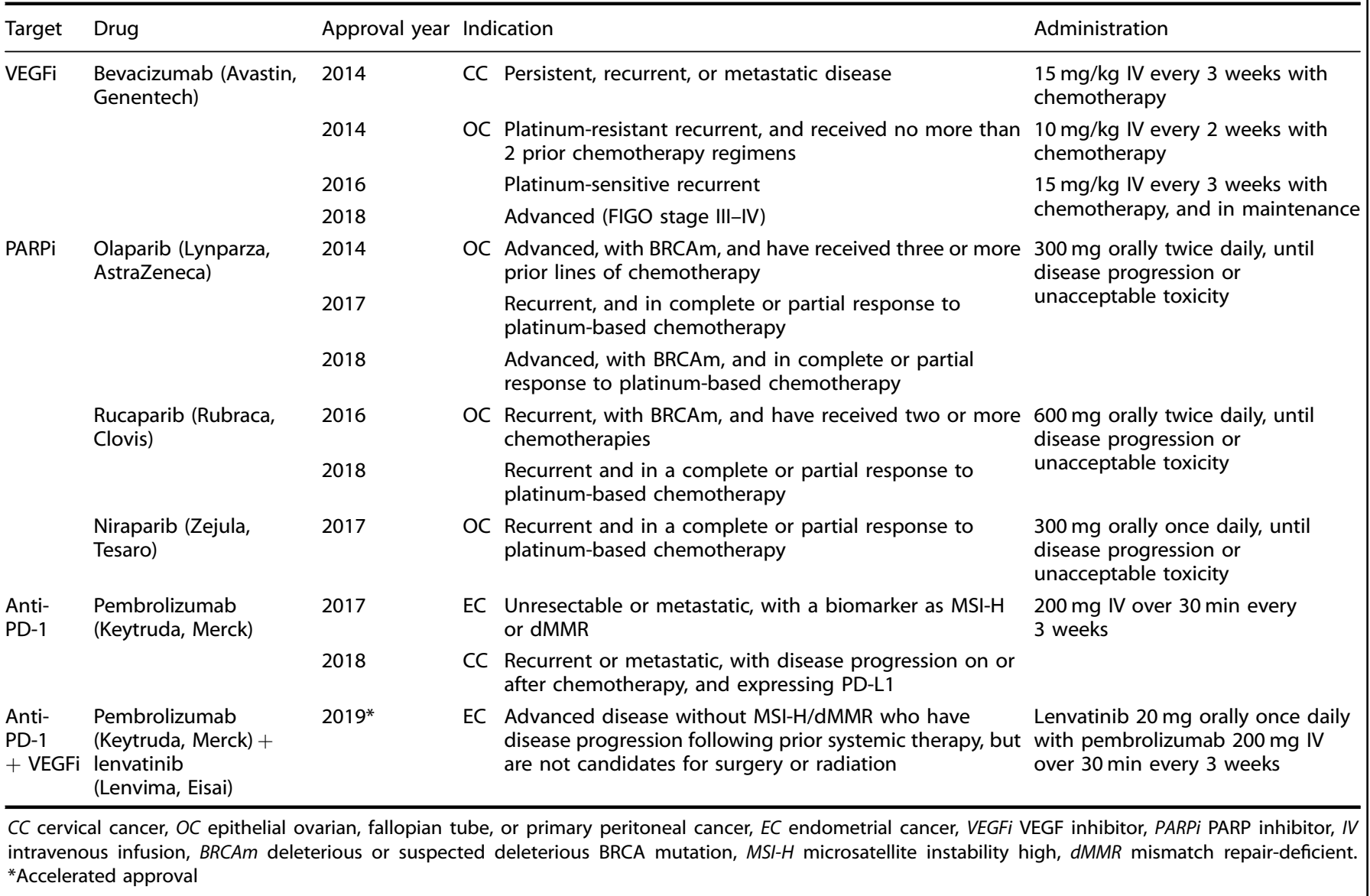

hormone receptors, and immunologic factors. The corresponding targeted agents include signaling pathway inhibitors, antiangiogenic agents, poly (ADP-ribose) polymerase (PARP) inhibitors, selective estrogen receptor downregulators, and Immune checkpoint inhibitors. For gynecological cancers, bevacizumab, olaparib, rucaparib, niraparib, and pembrolizumab have been approved by the US Food and Drug Administration (FDA) for selected patients with recurrent, metastatic, or high-risk diseases (Table 1). The clinical uses of these and other targeted agents are being actively and extensively investigated.

In this paper, we review the clinical efficacy and safety of the targeted therapies in gynecological cancers, by summarizing the results of previous clinical trials. We further describe the ongoing phase II/III clinical trials and expound future directions.

\section{METHODS}

A comprehensive literature review was performed on PubMed, including systematic reviews, review articles, clinical trials, and observation studies published in English. ClinicalTrials.gov was queried to collect the data of completed and ongoing clinical trials. For each approved targeted drug, the FDA website was searched for indication, usage and references as the basis for approval. Search terms included "gynecological cancers", "ovarian cancer", "cervical cancer", "endometrial cancer", "targeted therapy", "antiangiogenic agents", "PARP inhibitor", "signaling pathway inhibitors", "immune checkpoint inhibitors", and each name of the targeted agent (e.g., "bevacizumab", "olaparib"). We also used the ESMO and ASCO websites for preliminary results reported from ongoing trials.
Antiangiogenic agents

Neovasculature is considered as a crucial process for tumor growth and progression. ${ }^{22}$ In decades, efforts have been made to develop vascular-targeted therapies for cancer treatment. Depending on the distinctly different mechanisms, vasculartargeted therapies include antiangiogenic agents and vasculardisrupting agents. ${ }^{23}$ Here, we focus on the action of antiangiogenic agents in this review.

Angiogenesis is a complex process regulated by various proangiogenic and antiangiogenic factors. ${ }^{24}$ Vascular endothelial growth factor (VEGF), a major driver of angiogenesis in solid tumors, binds to the VEGF receptors (VEGFR, including VEGFR-1/2/ 3 ) on target cells and initiates the signaling pathway through intracellular tyrosine kinases. ${ }^{25}$ It can initiate several endothelial cell signaling pathways and promote endothelial cell precursors from bone marrow. ${ }^{24}$ The VEGF pathway also interacts with the $\mathrm{PI} 3 \mathrm{~K} / \mathrm{AKT} / \mathrm{mTOR}$ pathway. ${ }^{26,27}$ Moreover, the process of angiogenesis is further modulated by the platelet-derived growth factor (PDGF) pathway, the fibroblast growth factor (FGF) pathway, the epidermal growth factor (EGF) pathway, and the angiopoietin family and their receptor tyrosine kinase (Tie2) pathways. ${ }^{28}$ There are complicated interplays of these pro-angiogenic pathways (Fig. 1). ${ }^{29}$ In addition, the VEGF expression can be induced by hypoxiaassociated transcription factors, such as hypoxia inducible factors (HIF1A and HIF2A). It is also associated with other genetic alterations such as TP53, RAS, and EGFR. ${ }^{30}$

In tumor cells, the expression levels of the pro-angiogenic factors, especially VEGF, are upregulated to develop tumor's own endogenous blood vessels, which is associated with the poor prognosis. ${ }^{22,31}$ Therefore, antiangiogenic therapies are developed 


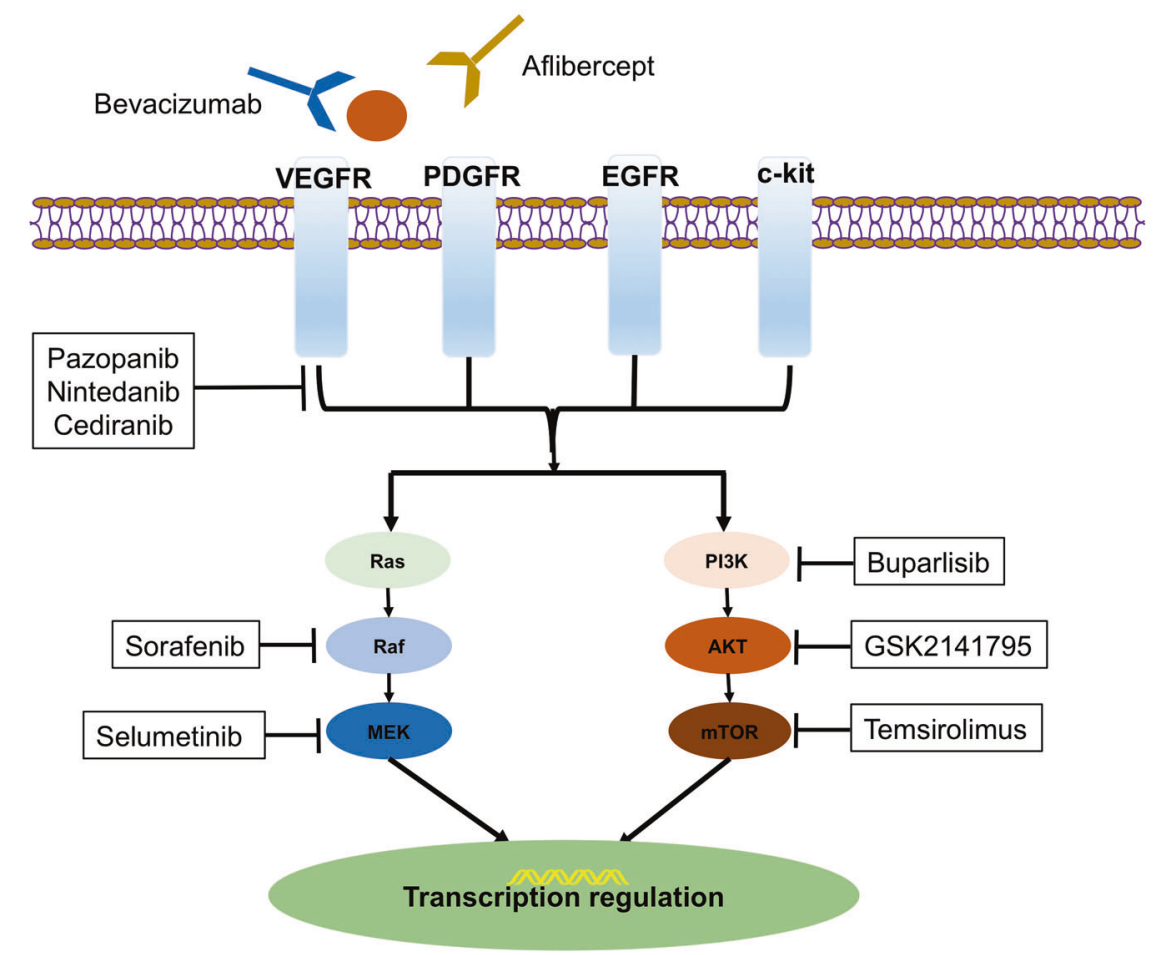

Fig. 1 The VEGF, PI3K/AKT/mTOR, and Ras/Raf/MEK signal transduction pathway and therapeutic interventions. After ligand binding, the receptors initiate the signaling cascade reaction, which is overactive in cancer cells. The figure shows the main elements in those pathways and the therapeutic agents

by inhibiting target signaling pathways at different points. The main classes of antiangiogenic agents are anti-VEGF monoclonal antibodies (e.g., bevacizumab), soluble VEGFRs (e.g., aflibercept), inhibitors of angiopoietin-Tie2 receptor (e.g., trebananib), and tyrosine kinase inhibitors (e.g., cediranib). ${ }^{24,32}$ Tyrosine kinases are enzymes that catalyze the transfer of phosphate from adenosine triphosphate (ATP) onto target proteins to elicit a response. ${ }^{33}$ Tyrosine kinase inhibitors (TKIs) are small molecules which can block intracellular tyrosine kinases in multiple signaling pathways (e.g., VEGF, EGF).

A number of antiangiogenic agents, such as bevacizumab, pazopanib, sunitinib, sorafenib, vandetanib, aflibercept, axitinib, regorafenib, ramucirumab, and lenvatinib are FDA-approved for cancer treatment (e.g., colorectal cancer, lung cancer, renal cell carcinoma, and thyroid cancer). For gynecological cancers, bevacizumab was the first and only FDA-approved anti-VEGF drug. As of January 2020, there are a dozen of completed phase III trials assessing the efficacy and safety of antiangiogenetic agents for gynecological cancers, especially in OC. The main data from completed Phase II/III clinical trials are summarized in Tables 2 and 3.

\section{Bevacizumab}

Bevacizumab is a humanized anti-VEGF monoclonal antibody, which is the best-known antiangiogenetic agent. In gynecological cancers, bevacizumab is currently approved by FDA as combination treatment and/or maintenance treatment for selected patients with: (1) persistent, recurrent, or metastatic CC; (2) advanced or recurrent OC (including stage III/IV epithelial ovarian cancer, fallopian tube, or primary peritoneal cancer) (Table 1). The decisions of these indications are mainly grounded on findings from the following six Phase III clinical trials (five for OC and one for CC) (Table 2).

GOG-0218 trial (NCT00262847) evaluated the efficacy of bevacizumab ( $15 \mathrm{mg} / \mathrm{kg}$ intravenously every 3 weeks) in combination with chemotherapy plus/without bevacizumab maintenance for patients with newly diagnosed advanced $O C$ following initial surgery. The median progression-free survival (PFS) was increased in the bevacizumab-concurrent plus maintenance arm when compared with control (chemotherapy alone) arm (3.8 months longer, $P<0.001$ ). PFS was not significantly increased in the bevacizumab-concurrent arm (without bevacizumab maintenance). ${ }^{34}$ However, final results of this trial were updated in July, 2019. When compared with the control arm, there is no significant increase in the median OS either in the bevacizumab-concurrent plus maintenance arm or in the bevacizumab-concurrent arm. In a subset analysis stratified by stage, for patients with stage IV disease, the control and bevacizumab-concurrent arms were associated with a median OS of 32.6 and 34.5 months, respectively. The median OS was increased in patients with stage IV disease who received bevacizumab-concurrent plus maintenance (42.8 months, HR, 0.75; 95\% Cl, 0.59-0.95). ${ }^{35}$ Another phase III trial, ICON7 (NCT00483782) found a modest increase in the median PFS (2.4 months longer, $P=0.25$ ) with no OS benefit in chemotherapy plus bevacizumab (both concurrence and maintenance) arm in the updated analyses. ${ }^{36}$ However, in a subset analysis of patients at high risk of progression, a significant difference in the median OS was noted between patients in chemotherapy plus bevacizumab arm and those in chemotherapy alone arm (39.3 vs. 34.5 months, $P=0.03) .{ }^{37}$ Data from these two trials did not show a statistically different quality of life (QOL) in the whole study population. ${ }^{38}$ Owing to the above trials, the FDA approved bevacizumab in combination with chemotherapy and followed as maintenance therapy for newly diagnosed advanced OC patients after initial surgical resection.

For patients with platinum-sensitive recurrent OC, OCEANS trial (NCT00434642) showed that the median PFS was significantly increased (4 months longer, $P<0.0001)$ in chemotherapy plus bevacizumab arm compared with chemotherapy alone. ${ }^{39}$ However, no significant difference in OS was observed at the final analysis. ${ }^{40}$ On the other hand, another phase III trial GOG-0213 (NCT00565851) showed that the addition of bevacizumab to chemotherapy led to a significant difference in both median PFS 
Table 2. Completed phase III trials of antiangiogenic agents in gynecological cancers

\begin{tabular}{|c|c|c|c|c|c|c|c|}
\hline ID & Cancer/condition & No. & Intervention & mPFS (mon.) & mOS (mon.) & SAEs (\%) & Refs \\
\hline & $\begin{array}{l}\text { OC/high-risk stage I-Ila, } \\
\text { Ilb-IV }\end{array}$ & 1528 & (2) PC + bevacizumab & $19.9, P=0.25$ & $58.0, P=0.85$ & - & 37 \\
\hline & $\begin{array}{l}\text { OC/platinum-resistant } \\
\text { recurrent }\end{array}$ & 361 & $\begin{array}{l}\text { (2) Chemotherapy }+ \\
\text { bevacizumab }\end{array}$ & $6.7, P<0.001$ & $16.6, P=0.174$ & 31.28 & 42 \\
\hline NCT00434642 OCEANS & $\begin{array}{l}\text { OC/platinum-sensitive } \\
\text { recurrent }\end{array}$ & 484 & (1) GC + placebo & 8.4 & 32.9 & 25.32 & 40 \\
\hline \multirow[t]{3}{*}{ NCT00262847 GOG-0218 } & \multirow[t]{3}{*}{ OC/stage III-IV } & \multirow[t]{3}{*}{1873} & (1) $\mathrm{PC}+$ placebo & 10.3 & 41.1 & 38.49 & \multirow[t]{3}{*}{35} \\
\hline & & & $\begin{array}{l}\text { (2) } P C+\text { bevacizumab } \\
\text { throughout }\end{array}$ & $14.1, P<0.001$ & $40.8, P=0.34$ & 41.19 & \\
\hline & & & $\begin{array}{l}\text { (3) } \mathrm{PC}+\text { bevacizumab } \\
\text { combination only }\end{array}$ & $11.2, P=0.16$ & $43.4, P=0.53$ & 46.37 & \\
\hline NCT00565851 GOG-0213 & $\begin{array}{l}\text { OC/platinum-sensitive } \\
\text { recurrent }\end{array}$ & 674 & (1) $\mathrm{PC}$ & 10.4 & 37.3 & 86 & 41 \\
\hline & \multirow{2}{*}{$\begin{array}{l}\mathrm{CC} / \text { metastatic, persistent, or } \\
\text { recurrent }\end{array}$} & \multirow{2}{*}{452} & (3) $\mathrm{PC}+$ bevacizumab & & & 47.75 & \multirow{2}{*}{42,43} \\
\hline & & & (4) PT + bevacizumab & $8.2, P=0.002$ & $16.8, P=0.007$ & 55.96 & \\
\hline \multirow[t]{3}{*}{ NCT00532194 ICON6 } & \multirow{3}{*}{$\begin{array}{l}\text { OC/platinum-sensitive } \\
\text { recurrent }\end{array}$} & \multirow[t]{3}{*}{486} & (1) Chemotherapy + placebo & 8.7 & - & - & \multirow[t]{3}{*}{73} \\
\hline & & & $\begin{array}{l}\text { (2) Chemotherapy + cediranib } \\
\text { throughout }\end{array}$ & 9.9 & & - & \\
\hline & & & $\begin{array}{l}\text { (3) Chemotherapy + cediranib } \\
\text { combination only }\end{array}$ & $11, P<0.0001$ & & - & \\
\hline \multirow[t]{2}{*}{ NCT01015118 AGO-OVAR12 } & \multirow[t]{2}{*}{ OC/stage Illb-IV } & \multirow[t]{2}{*}{1503} & (1) $\mathrm{PC}+$ placebo & 16.6 & 62.8 & 34.89 & 67 \\
\hline & & & (2) $\mathrm{PC}+$ nintedanib & $17.2, P=0.24$ & $62, P=0.087$ & 42.02 & \\
\hline NCT00866697 AGO-OVR16 & OC/stage II-IV, after first- & 940 & (1) Placebo & 12.3 & 64.0 & 11.06 & 63 \\
\hline & line $c$ & & (2) Pazopanib & $17.9, P=0.0021$ & $59.1, P=0.64$ & 25.37 & \\
\hline
\end{tabular}

ID identifier, No. enrollment number, mPFS median progression-free survival, mOS median overall survival, Mon. months, SAEs serious adverse events, Refs references, Stage FIGO stage, PC paclitaxel + carboplatin, GC gemcitabine + carboplatin, PT topotecan + paclitaxel, PLD pegylated liposomal doxorubicin

(3.4 months longer, $P<0.0001)$ and OS (4.9 months longer, adjusted $P=0.0447$ ) in patients with platinum-sensitive recurrent OC. $^{41}$ The FDA approved bevacizumab in combination with firstline chemotherapy and followed as maintenance therapy for platinum-sensitive recurrent OC patients in 2016.

For patients with platinum-resistant recurrent $\mathrm{OC}$, an open-label phase III trial, AURELIA (NCT00976911), found that the addition of bevacizumab to chemotherapy improved the median PFS (3.3 months longer, $P<0.001$ ), but with no benefit in OS at the final analysis. ${ }^{42,43}$ Based on this trail, the FDA approved bevacizumab in combination with chemotherapy for platinumresistant recurrent $\mathrm{OC}$ patients who received no more than two prior chemotherapy regimens.

Another phase III trial (NCT01081262), studying different chemotherapy regimens with or without bevacizumab as the first-line therapy in treating patients with mucinous epithelial $O C$, was closed early due to slow accrual. ${ }^{44}$ An ongoing phase III trial (NCT03635489) is evaluating the efficacy and safety of bevacizumab plus chemotherapy in Chinese participants with newly diagnosed advanced OC.
For CC, phase II trials (e.g., NCT00548418) demonstrated that the combination of chemotherapy and bevacizumab in patients with recurrent or persistent CC had an objective response rate (ORR) of $59-88 \% .{ }^{45-47}$ Furthermore, a phase III trial, GOG-0240 (NCT00803062), revealed an improvement in the median PFS (2.2 months longer, $P=0.0002)$ and OS (3.5 months longer, $P=$ 0.007 ) among patients receiving chemotherapy plus bevacizumab compared with those receiving chemotherapy alone. ${ }^{48}$ Based on this trail, the FDA approved bevacizumab in combination with standard chemotherapy for metastatic, persistent, or recurrent CC. For locally advanced CC, a phase II trial (NCT00369122) showed concurrent cisplatin-based chemoradiotherapy and bevacizumab had an ORR of $68.7 \% .{ }^{49}$ Another phase II/III trial (JCOG1311) has been initiated to compare different chemotherapy regimens with or without bevacizumab in stage IVb, recurrent or persistent CC. ${ }^{50}$

Currently, there are limited results of phase III studies assessing the efficacy of bevacizumab for patients with EC. In a phase II trial (NCT00301964) for persistent or recurrent EC, the single-agent bevacizumab therapy was shown to have an ORR of $13.5 \%$, with the median PFS and OS being 4.2 and 10.5 months, respectively. ${ }^{51}$ Another phase II trial (NCT00879359) for advanced or recurrent EC 


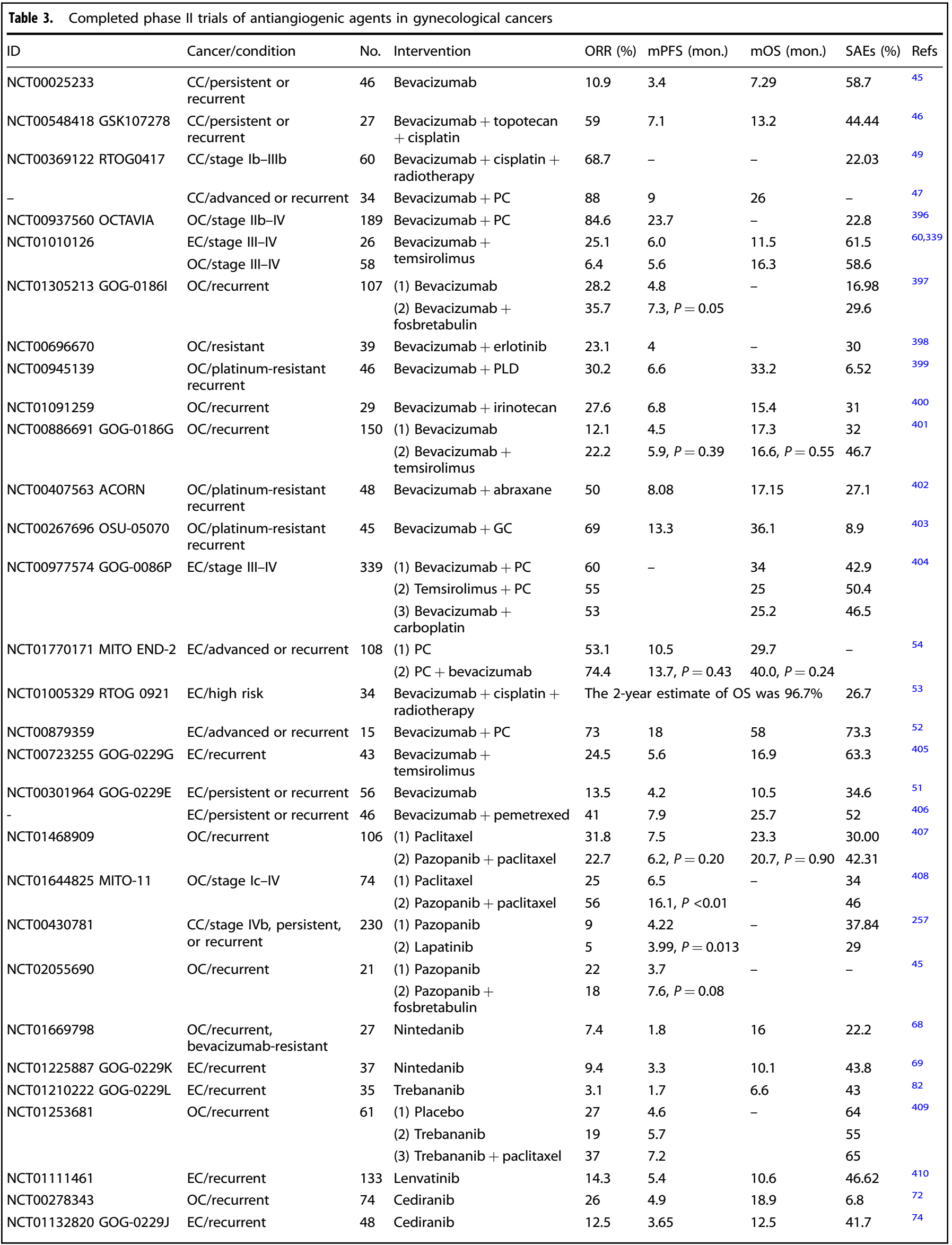




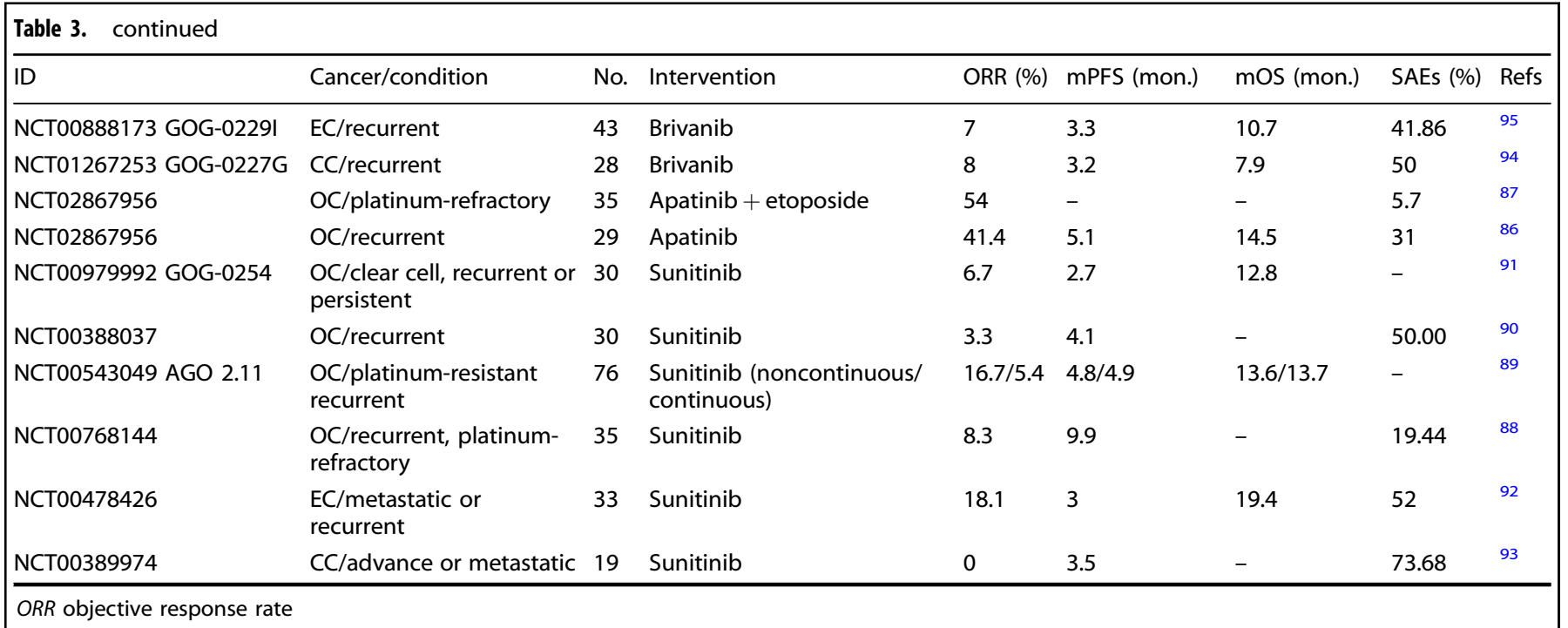

showed that bevacizumab in combination with chemotherapy had an ORR of $73 \%$, presenting a median PFS of 18 months and a median OS of 58 months. ${ }^{52}$ For patients with high-risk EC, postoperative bevacizumab added to chemotherapy and pelvic radiotherapy resulted in a high OS rate (at 2 years) of $96.7 \%$ and a disease-free survival rate of $79.1 \%$, which was reported in a phase II trial (NCT01005329). ${ }^{53}$ However, bevacizumab plus chemotherapy failed to demonstrate a significant increase in PFS of patients with advanced or recurrent EC, reported by the MITO END-2 trial (NCT01770171) in 2019. ${ }^{54}$

Grade 3 or worse adverse events (AEs) occurring at a higher incidence (incidence $\geq 2 \%$ ) in patients receiving chemotherapy plus bevacizumab compared with chemotherapy alone (from data of those phase III trials) included fatigue, hypertension, neutropenia, thrombocytopenia, proteinuria, nausea, headache, dyspnea, epistaxis, abdominal pain, hyponatremia, pain in extremity, and palmar-plantar erythrodysaesthesia syndrome. ${ }^{55}$

\section{Pazopanib}

Pazopanib is an oral TKI of VEGFR-1/-2/-3, PDGF receptor (PDGFR) $-\alpha /-\beta$, and $c-K i t .{ }^{56-58}$ Pazopanib showed promising activity in phase $\mathrm{I} / \mathrm{II}$ trials for patients with platinum-sensitive recurrent OC with increased ORR and PFS. ${ }^{59-61}$ A phase III trial, AGO-OVAR16 (NCT00866697), investigated the efficacy and safety of pazopanib (800 mg daily) as maintenance therapy after first-line chemotherapy in patients with newly diagnosed stage II-IV OC. The study showed that the pazopanib maintenance significantly improved the median PFS (5.6 months longer, $P=0.0021){ }^{62}$ In subgroup analyses, the PFS benefit with maintenance pazopanib was observed in most subgroups except East Asian patients. To gain further insight, a concurrent study (NCT01227928) similar in design to AGO-OVAR16 was undertaken in the East Asian population, showing that pazopanib maintenance therapy was not associated with a benefit in PFS or OS. There was no satisfactory explanation for this result yet. However, the final analysis of the OVAR16 study was reported in 2019. No difference was observed in the median OS between pazopanib arm and placebo arm. ${ }^{63}$ Grade 3 or worse AEs occurring at a higher incidence in the combined treatment arm compared with placebo included hypertension, neutropenia, diarrhea, thrombocytopenia, increased alanine aminotransferase, and palmar-plantar erythrodysesthesia. A phase I/II trial (NCT02055690) recently reported that combination of pazopanib and fosbretabulin (a prodrug with vascular-disrupting activity) might potentially improve survival outcomes compared with pazopanib alone. ${ }^{64}$ However, this trial was prematurely stopped due to serious cardiac toxicity.

Currently, there are limited data of clinical trials investigating pazopanib for patients with CC or EC. A phase II trial evaluated pazopanib in the treatment of recurrent or persistent carcinosarcoma of the uterus with a result of no response. ${ }^{65}$

Nintedanib

Nintedanib is another oral TKI of VEGFR-1/-2/-3, FGF receptor (FGFR)-1/-2/-3, and PDGFR- $\alpha / \beta$. A phase II trial in platinumsensitive recurrent $O C$ patients showed an improvement in PFS rate in nintedanib maintenance arm than placebo arm $(16.3 \%$ vs. $5.0 \%, P=0.06) .{ }^{66}$ Subsequently, a phase III trial, AGO-OVAR12 (NCT01015118), investigated the combination of nintedanib (200 mg daily) with first-line chemotherapy in patients with newly diagnosed stage IIb-IV OC. The median PFS was 0.6 month longer in the nintedanib arm than that in the placebo arm $(P=0.024){ }^{67}$ Increased incidences of AEs, including hypertension, gastrointestinal perforation, and bleeding, were reported in the nintedanib arm. The final result of OS is pending. However, for bevacizumabresistant OC population, single-agent nintedanib was shown to have minimal activity with an ORR of $7.4 \%$ in a phase II trial (NCT01669798). ${ }^{68}$

We found limited clinical data of phase II/III trials investigating the activity of nintedanib in EC and CC. One phase II trial, GOG0229K (NCT01225887), evaluated nintedanib in the treatment of advanced, recurrent, or metastatic EC. It showed modest activity with an ORR of $9.4 \% .^{69}$

\section{Cediranib}

Cediranib is a TKI of VEGFR-1/-2/-3 and c-Kit. ${ }^{70,71}$ Given the activity of cediranib in OC showed by early-phase trials, ${ }^{72}$ a phase III trial, ICON6 (NCT00532194), investigated the combination of cediranib (20 mg orally daily) with chemotherapy and as maintenance treatment in patients with platinum-sensitive recurrent $O C$. The median PFS was 2.3 months longer in the cediranib maintenance arm than that in the placebo arm $(P<0.0001) .{ }^{73}$ The data of OS have not been updated. Currently, there are no differences in immature results of median OS across the arms. Increased incidences of diarrhea, neutropenia, hypertension, and voice changes were noted in arms with cediranib.

A phase II study, GOG 229J (NCT01132820), showed cediranib as a monotherapy treatment for recurrent or persistent EC was welltolerated, with a median PFS of 3.65 months and a median OS of 
12.5 months. ${ }^{74}$ Cediranib showed sufficient activity to warrant further investigation for recurrent EC. However, we found limited clinical data for patients with CC.

\section{Trebananib}

Trebananib is a peptide-Fc fusion protein that binds angiopoietin$1 /-2$, preventing the interaction of angiopoietin with the Tie2 receptor. ${ }^{75}$ Trebananib has shown single-agent activity and prolonged PFS in recurrent $\mathrm{OC}$ in early-phase trials. ${ }^{76,77}$ There are three completed phase III trials assessing trebananib in recurrent or newly diagnosed advanced OC. TRINOVA-1 trial (NCT01204749) investigated the addition of trebananib $(15 \mathrm{mg} / \mathrm{kg}$ intravenously weekly) to single-agent weekly paclitaxel in recurrent $O C$ with platinum-free interval $\leq 12$ months. As a result, the median PFS was 1.8 months longer in the trebananib arm than that in the placebo arm $(P<0.0001) .{ }^{78}$ Subsequently, TRINOVA-2 (NCT0128125) evaluated the addition of trebananib to PLD in patients with recurrent $O C$, and it showed that trebananib did not significantly prolong PFS. However, the addition of trebananib to PLD improved ORR compared with placebo arm (46\% vs. $21 \%, P<$ $0.001) .{ }^{79}$ TRINOVA-3 trial (NCT01493505) showed that the addition of trebananib to fist-line chemotherapy did not improve PFS or produce new safety signals for patients with newly diagnosed advanced $O C^{80}$ The result of OS was not mature. The major toxic effect associated with trebananib treatment was edema. ${ }^{8,81}$

For recurrent or persistent EC, a phase II trial (NCT01210222) showed an ORR of $3.1 \%$, with insufficient single-agent activity to warrant further investigation of trebananib. ${ }^{82}$

Other antiangiogenic agents

Apatinib is a small-molecule TKI by binding to the VEGFR-2 ATPbinding site, which is taken orally. ${ }^{83,84}$ Given the promising results of a phase III study in Chinese gastric cancer patients, ${ }^{85}$ apatinib had been actively investigated as a salvage treatment for other advanced solid tumor, including $O C^{84}$ A phase II study of apatinib in patients with recurrent $O C$ indicated that apatinib $(500 \mathrm{mg}$ daily) was a feasible treatment with an ORR of $41.4 \% .{ }^{86}$ Grade 3 AEs were hand-foot syndrome, hypertension, and neutropenia. Another phase II trial (NCT02867956) demonstrated that apatinib plus etoposide showed promising efficacy and manageable toxicities in patients with platinum-resistant or -refractory $O C$ with an ORR of $54 \% .^{87}$ An ongoing phase III trial in China (NCT04000295) is further evaluating the efficacy and safety of apatinib in patients with platinum-resistant recurrent OC compared with chemotherapy.
Sunitinib and brivanib are oral TKIs of VEGFR and PDGFR. Sunitinib was an FDA-approved drug for renal cell cancer and gastrointestinal stromal tumors. The safety and efficacy of sunitinib in OC were evaluated in several phase II trials with reported ORR ranging from $3.3 \%$ to $16.7 \% .^{88-91}$ In metastatic or recurrent EC, sunitinib showed promising activity in a phase II trial (NCT00478426) with an ORR of $18.1 \% .^{92}$ However, sunitinib had insufficient activity as a single agent in advanced or metastatic CC to warrant further investigation. ${ }^{93}$ Two phase II trials demonstrated that brivanib was well-tolerated and worthy of further investigation in persistent or recurrent EC/CC with an ORR of $7 \%$ and $8 \%$, respectively. ${ }^{94,95}$

For the development of antiangiogenic agents and other targeted therapies, the addition of bevacizumab to conventional chemotherapy in $\mathrm{OC}$ is a very important step. However, most of the analysis reported so far showed that antiangiogenic agents led to no significant improvement in OS for patients with gynecological cancers. Thus, identification of predictive biomarkers for antiangiogenic agents and development of other targeted drugs are anticipated.

Poly (ADP-ribose) polymerase (PARP) inhibitors PARP is a sort of nuclear enzyme with 17 identified members. ${ }^{96}$ PARP- 1 and 2 are involved in DNA repair. ${ }^{97}$ PARP-1, with a structure of the $\mathrm{N}$-terminal zinc-finger DNA-binding domain, the central automodification domain and the C-terminal catalytic domain, was originally found involved in the base-excision repair (BER) pathway, which is important in the repair of single-stranded DNA breaks (SSBs). ${ }^{98}$ Therefore, inhibition of PARP-1 leads to the accumulation of DNA SSBs and ultimately results in DNA doublestrand breaks (DSBs) during DNA replication. ${ }^{99}$ DSBs are the most lethal DNA insults. Nonhomologous end joining (NHEJ) and homologous-recombination repair (HRR) are the two main DSB repair pathways in humans. ${ }^{100}$ The preferred pathway is HRR, since it is more accurate. Thus, in cells with functional HRR, PARP inhibition will not result in cell death since DSBs will be precisely and effectively repaired. However, in cells with homologousrecombination deficiency (HRD), such as those with BRCA1/2 mutations, DSBs are left unrepaired or repaired by the error-prone NHEJ pathway, which result in genomic instability and ultimately cell death. ${ }^{101}$ This mechanism of synthetic lethality in HRD cells (Fig. 2) makes PARP inhibitors a novel targeted and personalized cancer treatment. ${ }^{102,103}$

In gynecological cancers, germline and somatic BRCA1/2 mutations (gBRCAm and sBRCAm) occur in $\sim 10-15 \%$ of OC

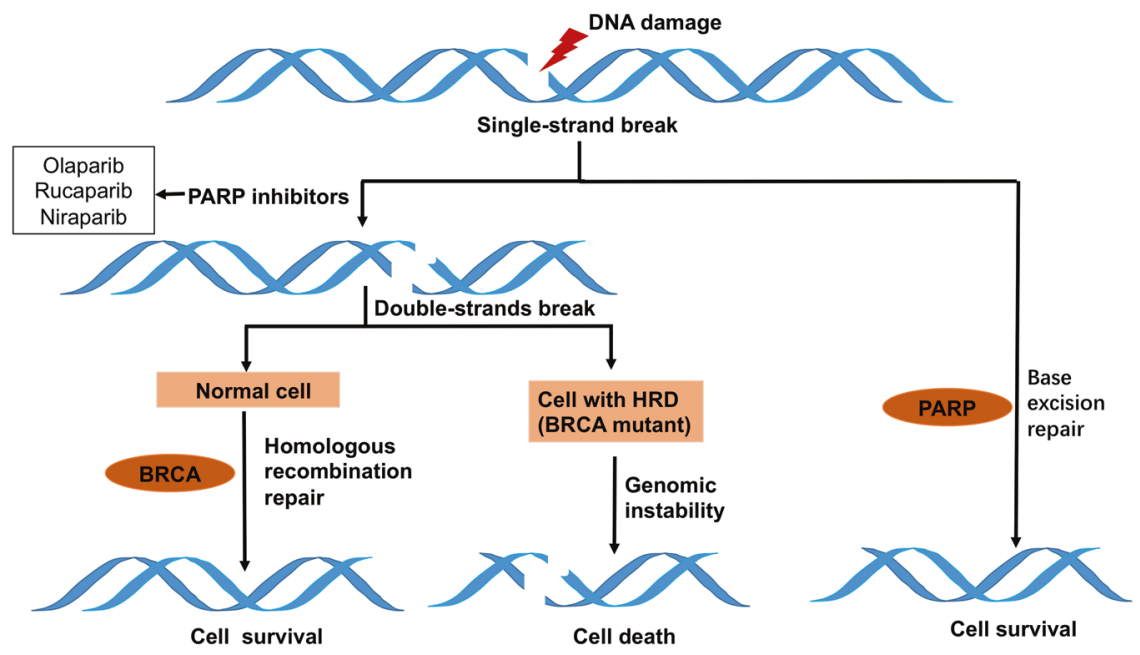

Fig. 2 Base-excision repair/single-strand break pathway and the mechanism of synthetic lethal interactions. Inhibition of PARP-1 causes the accumulation of DNA SSBs and ultimately results in DSBs during DNA replication. In cells with HRD, DSBs are left unrepaired or repaired by the error-prone NHEJ pathway, which result in genomic instability and ultimately cell death 
Table 4. Phase III trials (with results) of PARP inhibitors in gynecological cancers

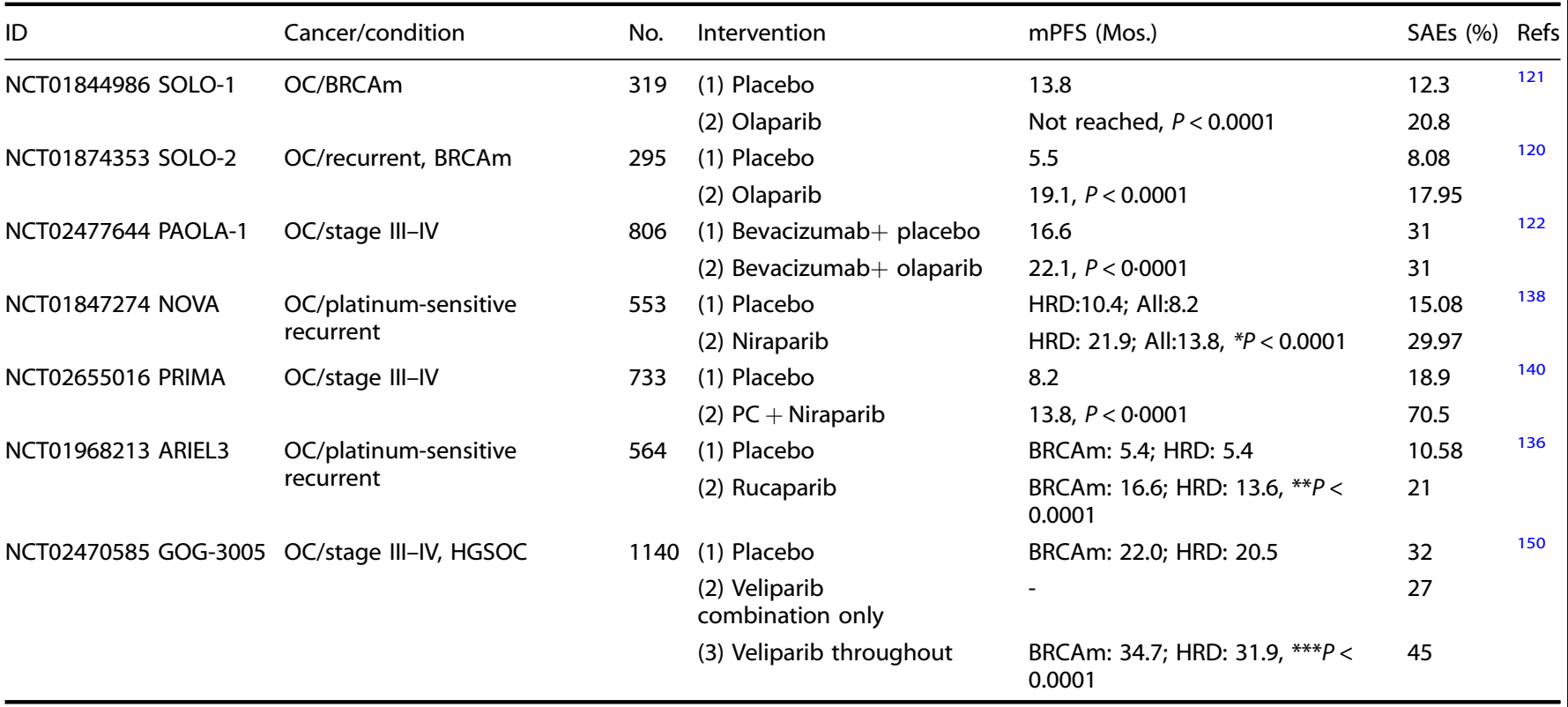

HRD homologous-recombination deficiency, HGSOC high-grade serous ovarian cancer. ${ }^{*} P$-value of both HRD cohort and all population are $<0.0001 .{ }^{* *}$ and ${ }^{* * *}$ $P$-value of both BRCAm and HRD cohorts are $<0.0001$

patients, and even more frequently in patients with high-grade serous OC (HGSOC), which is the most common type of $\mathrm{OC}^{22,104,105}$ In addition, genomic alterations in other homologous-recombination (HR) genes including ATM, BRIP1, PALB2, and RAD51C are being studied. ${ }^{106}$ The comprehensive genomic analysis has identified that $\sim 50 \%$ of high-grade serous tumors (including OC and EC) exhibit HRD. ${ }^{107,108}$ Moreover, the presence of HRD predicts a favorable response to platinum therapies and to PARP inhibitors. PARP inhibitors are also known to sensitize DNA-damaging agents, including carboplatin. ${ }^{109}$ Based on the above facts, PARP inhibitors are supposed to be groundbreaking therapeutic strategies for patients with gynecological cancers, especially for OC. ${ }^{110}$

Several PARP inhibitors, including olaparib, rucaparib, niraparib, veliparib, and talazoparib are actively investigated in clinical trials. The development of PARP inhibitors is productive. Olaparib is the first PARP inhibitor applied in clinic and approved by FDA for cancer treatment, followed by rucaparib and niraparib. The results from phase II/III clinical trials, assessing PARP inhibitors in gynecological cancers, are summarized in Tables 4 and 5 . The ongoing clinical trials without results are listed in Table 6.

\section{Olaparib}

Olaparib is the best studied PARP inhibitor and approved by FDA for the maintenance treatment of selected advanced or recurrent OC patients. Early-phase clinical trials of olaparib demonstrated activity signals in patients with $O C$, with favorable tolerance and response rates. ${ }^{58,111-113}$ Following these promising results, ${ }^{114} \mathrm{a}$ notable randomized placebo-controlled phase II trial, Study 19 (NCT00753545), evaluated olaparib as maintenance monotherapy for patients with platinum-sensitive recurrent OC. The median PFS was significantly longer in the olaparib arm compared with placebo (3.6 months longer, $P<0.001) .{ }^{115} \mathrm{~A}$ retrospective preplanned analysis suggested that patients with BRCAm gained the greatest PFS benefits from olaparib treatment (6.9 months longer, $P<0.0001$ ). An exploratory post hoc analysis of Study 19 also suggested a numerical improvement in the OS. ${ }^{116}$ Although the PFS benefit was less in patients without BRCAm (1.9 months longer, $P=0.0075)$, this significant benefit suggested that a proportion of patients without BRCAm might also benefit from olaparib treatment. ${ }^{117}$ Another single-arm phase II trial, Study 42 (NCT01078662), evaluated olaparib as treatment for cancer patients with gBRCAm, including ovarian, breast, prostate, and pancreatic cancer. The ORR was $31.1 \%$ in platinum-resistant recurrent OC cohort. Stable disease (SD) was seen in $40 \%$ of patients, confirming significant activity. ${ }^{118,119}$ Based on these findings, the FDA approved single-agent olaparib as recurrence therapy for patients with advanced OC with gBRCAm who have received three or more lines of chemotherapy in 2014.

Several large randomized phase III trials of olaparib in gynecological cancers (mainly in OC) are currently in progress. The following three of the phase III trials reported promising results in OC. SOLO-2 trial (NCT01874353) evaluated the efficacy of olaparib as maintenance therapy in platinum-sensitive recurrent OC patients with BRCAm who had received at least two lines of previous chemotherapy. The results demonstrated a statistically significant improvement in investigator-assessed median PFS in the olaparib arm compared with placebo (13.6 months longer, $P<$ $0.0001)$. At the time of the analysis of PFS, OS data were not mature with $24 \%$ of events. ${ }^{120}$ Based on this trial, the FDA approved olaparib as maintenance therapy for women with recurrent $O C$ who are in complete or partial response to platinumbased chemotherapy in 2017. Another phase III trial, SOLO-1 (NCT01844986), evaluated the efficacy of olaparib as maintenance therapy in newly diagnosed advanced OC patients with BRCAm. ${ }^{121}$ After a median follow-up of 41 months, the risk of disease progression or death was $70 \%$ lower with olaparib than with placebo $(P<0.001)$. The estimated median PFS was not reached in the olaparib arm versus 13.8 months in the placebo arm $(P<$ $0.0001)$. At the time of the analysis, OS data were not mature. Following this study, the FDA approved olaparib as maintenance therapy of advanced OC patients with BRCAm, who are in complete or partial response to first-line platinum-based chemotherapy in 2018. At the ESMO Congress 2019, new findings of a phase III trial, PAOLA-1/ENGOT-ov25 (NCT02477644), were presented. This is the first phase III trial to evaluate efficacy and safety of a PARP inhibitor plus bevacizumab as first-line maintenance therapy in advanced OC not restricted by surgical outcome or 


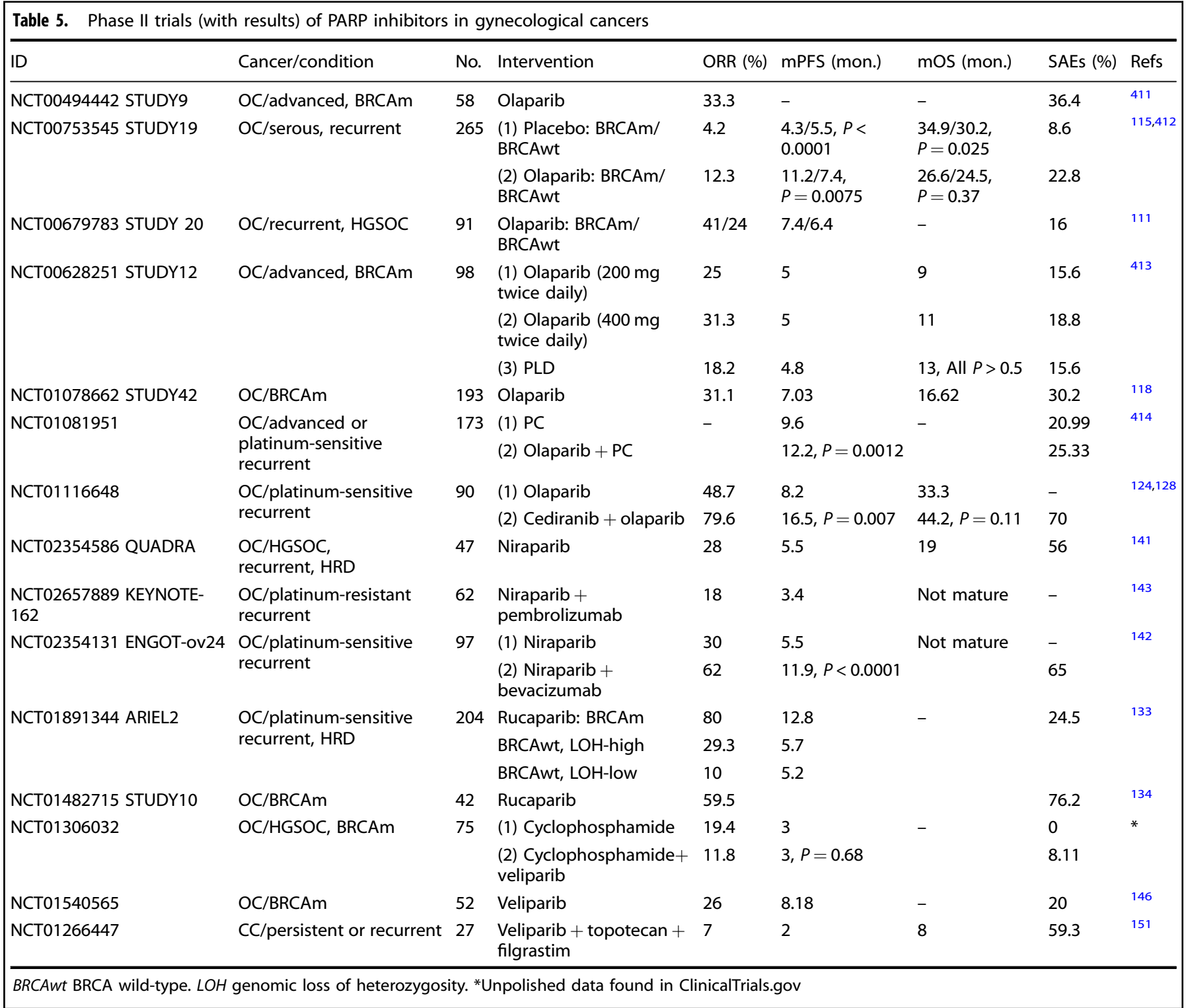

BRCA status. According to the results, patients with newly diagnosed OC had significantly improved the median PFS with addition of olaparib to bevacizumab maintenance treatment, as compared to placebo plus bevacizumab following first-line chemotherapy $(5.5$ months longer, $P<0.0001))^{122}$ Moreover, the PFS benefit in subgroups of patients with BRCAm and patients with other HRD was even more obvious (19.5 months longer and 11.5 months longer, respectively). In PAOLA- 1 trial, the rate of AEs leading to treatment discontinuation is the highest figure reported across PARP inhibitor trials. However, there was no impact in QOL.

The FDA-recommended olaparib dose is $300 \mathrm{mg}$ (two $150 \mathrm{mg}$ tablets) taken orally twice daily. The most common serious AEs reported in SOLO-1 and SOLO-2 were anemia and neutropenia.

There are other three ongoing phase III trials of olaparib (as monotherapy) registered in the ClinicalTrials.gov database without available results, including SOLO-3 (NCT02282020), OPINION (NCT03402841), and L-MOCA (NCT03534453) (Table 6).

A phase II trial (NCT01116648) evaluated the efficacy and toxicity of the combination of cediranib and olaparib compared to olaparib alone in platinum-sensitive recurrent $O C$, based on the data from early clinical trial. ${ }^{123-126}$ This novel combination of angiogenesis inhibitor and PARP inhibitor improved the median PFS by 8.3 months compared with PARP inhibitor alone $(P=$ 0.007). ${ }^{124,127}$ In the updated analysis in 2019, subset analyses within stratum defined by BRCA status demonstrated that this combination therapy significantly improved both median PFS (23.7 vs. 5.7 months, $P=0.002)$ and median OS $(37.8$ vs. 23.0 months, $P=0.047$ ) in gBRCAwt/unknown patients. ${ }^{128}$ It encouraged the novel combination therapy of different targeted agents explored as a potential treatment strategy. Currently, we found only clinical case reports about efficacy of olaparib in other gynecological cancers (e.g., EC). ${ }^{129}$

Rucaparib

Rucaparib is a potent, oral, small-molecule PARP inhibitor. ${ }^{130,131}$ Rucaparib was FDA-approved in 2016 as monotherapy for the treatment of recurrent OC patients with BRCAm who have been treated with two or more chemotherapies. This approval was grounded on the proportion of patients with a favorable ORR observed in a pooled population of patients with BRCAm highgrade OC from the Study 10 and ARIEL2 trials. ${ }^{132-135}$ ARIEL2 (NCT01891344) is a phase II trial, assessing rucaparib as recurrence 
therapy for patients with platinum-sensitive OC. The median PFS after rucaparib treatment was 7.6 months longer in the BRCAm subgroup $(P<0.0001)$.

In a phase III trial, ARIEL3 (NCT01968213), assessed the efficacy and safety of rucaparib as maintenance therapy in patients with platinum-sensitive recurrent OC. The median PFS in patients with BRCAm was 11.2 months longer in the rucaparib arm than that in the placebo arm $(P<0.0001)$. In patients with HRD, it was 8.2 months longer $(P<0.0001)$. In the intention-to-treat (ITT) population, the median PFS was 5.4 months longer in patients in the rucaparib arm than that in the placebo arm $(P<0.0001) .^{136}$ Based on this study, the FDA approved rucaparib for the maintenance treatment of recurrent $O C$ patients who are in a complete or partial response to platinum-based chemotherapy. The ongoing ARIEL4 trial (NCT02855944) is another phase III study of rucaparib compared with chemotherapy in recurrent OC patients with BRCAm after two or more prior lines of therapy. The combination of rucaparib with other novel therapies (e.g., immune checkpoint inhibitor) is investigated for OC and EC in Phase I/II trials (NCT03101280, NCT03572478). A new phase III trial, MAMOC (NCT04227522), is going to investigate rucaparib maintenance therapy after bevacizumab maintenance following firstline chemotherapy in advanced OC.

The FDA-recommended rucaparib dose is $600 \mathrm{mg}$ (two $300 \mathrm{mg}$ tablets) taken orally twice daily. The most common serious AEs reported in ARIEL3 were anemia, pyrexia, vomiting, and small intestinal obstruction.

\section{Niraparib}

Niraparib is another FDA-approved PARP inhibitor. ${ }^{137}$ A phase III trial, ENGOT-OV16/NOVA (NCT01847274), evaluated the efficacy of niraparib as maintenance treatment for patients with platinumsensitive recurrent $O C$. The results showed that niraparib increased PFS regardless of BRCA status when compared with placebo. Patients in the niraparib arm had significantly longer median PFS than those in the placebo arm, including $21.0 \mathrm{vs.}$ 5.5 months in the gBRCAm cohort, 12.9 months vs. 3.8 months in the non-gBRCAm cohort for patients who had tumors with HRD, and 9.3 months vs. 3.9 months in the overall non-gBRCAm cohort $\left(P<0.001\right.$ for all three comparisons). ${ }^{138}$ Based on this study, niraparib was approved by FDA in 2017 as maintenance therapy for adult patients with recurrent $\mathrm{OC}$ who are in complete or partial response to platinum-based chemotherapy. ${ }^{138}$ Furthermore, A retrospective subanalysis demonstrated the safety and efficacy of niraparib in the subgroup of patients aged $\geq 70$ years in this trial, suggesting that the use of niraparib should be considered in this population. ${ }^{139}$ Findings from another phase III trial, PRIMA (NCT02655016), were presented at the ESMO Congress 2019, and recently reported. This study evaluated the efficacy of niraparib following first-line chemotherapy in patients with newly diagnosed advanced $\mathrm{OC}$ and had similar findings with NOVA trial. Patients in the niraparib arm had substantial improvement in the median PFS compared to those in placebo arm (5.6 months longer, $P<0.0001)$. In the HRD cohort, the improvement of the median PFS was even greater in treatment group (21.9 vs. 10.4 months, $P<0.0001){ }^{140}$ Another phase III trial (NCT03709316) of niraparib in advanced $O C$ is under way (Table 6). Several other phase II trials are studying the potential role of niraparib in different clinical settings. QUADRA trial (NCT02354586) assessed the activity of single-agent niraparib as the fourth or later line treatment for patients with platinum-sensitive recurrent HGSOC. ${ }^{141}$ This study met the primary endpoint, with an ORR of $28 \%$ in HRD-positive population. The median PFS in this population was 5.5 months. The median OS was 26 months in the BRCAm population, 19.0 months in the HRD-positive population, and 15.5 months in the HRD-negative population. NSGOAVANOVA2/ENGOT-OV24 trial (NCT02354131) showed that niraparib (300 mg orally daily) plus bevacizumab $(15 \mathrm{mg} / \mathrm{kg}$ intravenously every 3 weeks) significantly improved the median PFS compared with niraparib alone in patients with platinumsensitive recurrent $O C$ (5.4 months longer, $P<0.00001){ }^{142}$ TOPACIO/KEYNOTE-162 trial (NCT02657889) evaluated niraparib (200 mg orally daily) combined with pembrolizumab (an immune checkpoint inhibitor, 200mg intravenously on day 1 of each 21day cycle) in patients with recurrent OC. The ORR was $18 \%$, with a disease control rate of $65 \%$. This novel combination therapy was tolerable, and responses in patients without HRD were higher than expected with either agent as monotherapy. ${ }^{143}$

The FDA-recommended niraparib dose is $300 \mathrm{mg}$ taken orally once daily. The most common serious AEs reported in NOVA and PRIMA were thrombocytopenia, anemia, and neutropenia. Disutility analyses showed no significant QOL impairment associated with these toxic effects. ${ }^{144}$

\section{Veliparib}

Veliparib is a potent small-molecule inhibitor of PARP-1/2. ${ }^{145}$ Early-phase trials demonstrated activity of veliparib among OC patients with BRCAm to provide rationale for further clinical development. ${ }^{109,146-149} \mathrm{New}$ results from a phase III trial, VELIA/GOG-3005 (NCT02470585), were reported at the ESMO Congress 2019. It assessed the efficacy of veliparib $(150 \mathrm{mg}$ orally twice daily) added to first-line chemotherapy and continued as maintenance monotherapy in patients with previously untreated advanced HGSOC. In the BRCAm cohort, the median PFS was 12.7 months longer in the veliparibthroughout arm than in the control arm $(P<0.001)$. In the HRD cohort, it was 11.4 months longer $(P<0.001)$. And in the ITT population, the median PFS was 5.2 months longer $(P<0.001)$. AEs reported with veliparib were predominantly gastrointestinal and hematologic. The most common AE leading to the discontinuation of veliparib was nausea. ${ }^{150}$

For the treatment of $\mathrm{CC}$, there was a phase $\mathrm{I} / \mathrm{II}$ trial (NCT01266447) that assessed veliparib in combination with topotecan for patients with recurrent or persistent CC, showing minimal clinical activity with an ORR of $7 \% .{ }^{151}$ Another phase I trial (NCT01281852) investigated veliparib in combination with cisplatin and paclitaxel in patients with recurrent or metastatic CC. ${ }^{152}$ The results demonstrated an ORR of $34 \%$, illustrating the potential of PARP inhibitors as a combination therapy in CC.

Other PARP inhibitors

Talazoparib is a potent PARP inhibitor showing antitumor cytotoxicity at much lower concentrations than other agents, with an ORR of $42 \%$ in early-phase clinical trials for advanced OC with BRCAm. ${ }^{153,154}$

Pamiparib is a highly selective oral PARP-1/2 inhibitor capable of penetrating the brain. ${ }^{155}$ In a phase I trial of pamiparib combined with tislelizumab (an immune checkpoint inhibitor) in advanced solid tumors, $9(26 \%)$ of the 34 patients with OC achieved clinical responses. ${ }^{156}$ A phase II trial (NCT03933761) is assessing the clinical benefit rate of pamiparib in fusion-positive, reversion-negative HGSOC with BRCAm.

Fluzoparib is a novel PARP inhibitor undergoing clinical trials with potent anticancer activities. ${ }^{157,158}$ Two ongoing phase III trials (NCT03519230 and NCT03863860) are investigating the efficacy of pamiparib and fluzoparib as maintenance therapy in recurrent $\mathrm{OC}$, respectively.

In summary, PARP inhibitors are acting as an exciting new option for patients with OC by significantly increasing both PFS and OS, especially for those with HRDs. However, cost effectiveness and drug resistance remain to be improved. ${ }^{159,160}$ In the future, it is necessary to identify more indications and predictive biomarkers. ${ }^{161,162}$ Moreover, numerous ongoing clinical trials of novel combination therapies are guiding the future direction of targeted therapy strategies (Tables 13 and 14)..$^{163,164}$ 
12

$\mathrm{PI3K} / \mathrm{AKT} / \mathrm{mTOR}$ pathway blockade

The phosphatidylinositol 3-kinase/protein kinase $\mathrm{B} / \mathrm{mammalian}$ target of rapamycin (PI3K/AKT/mTOR) signaling is one of the critical intracellular pathways that regulates important cell activities, such as cell growth, survival, proliferation, differentiation, metabolism, apoptosis, and angiogenesis. ${ }^{165} \mathrm{PI} 3 \mathrm{~K}$ is plasma membrane-associated lipid kinases, composed of regulatory subunit (PIK3R) and catalytic subunit (PIK3CA) that mediate receptor binding, activation, and localization of the enzyme. ${ }^{166}$ In normal conditions, PI3K can be activated by a variety of stimuli, including growth factors, cytokines, and hormones. ${ }^{167}$ Activation of AKT regulates a number of downstream targets. mTOR is a serine/threonine protein kinase and the best-described downstream target of AKT, composed of mTOR Complex 1 (mTORC1) and mTOR Complex 2 (mTORC2). ${ }^{168}$ mTORC1 is sensitive to inhibition by rapamycin, and its analogs and mTORC2 exerts a positive feedback activation on AKT. ${ }^{169}$ There are also endogenous negative regulators of the PI3K pathway, such as the tumor suppressor-phosphatase and tensin homologue (PTEN). ${ }^{170}$ The $\mathrm{PISK} / \mathrm{Akt} / \mathrm{mTOR}$ pathway is also involved in cross talk with other signaling pathways, including the Ras/Raf/MEK and estrogen receptor (ER) pathways. ${ }^{171}$ The overview of the PI3K/AKT/mTOR signaling pathway is included in Fig. 1. In cancer, this pathway can be aberrantly activated via a number of mechanisms, including loss of tumor-suppressor function, exposure to carcinogens, mutations/amplifications of $\mathrm{PI} 3 \mathrm{~K}$, and mutations/amplifications of AKT. The deregulation of the PI3K/ AKT/mTOR pathway occurs in many cancers. ${ }^{172-174}$ As for gynecological cancers, this pathway is overactivated in OC $(\sim 70 \%),{ }^{175-177}$ as well as EC and CC. ${ }^{178-180}$ In $\mathrm{EC}$, the mutation rates of PI3K and PTEN were high, especially in the POLE subgroup. ${ }^{20}$ In vitro model of CC, mTOR inhibitors markedly reduced the expression level of HPV E7 protein, inducing apoptosis. ${ }^{181}$ Based on the preclinical evidence, the PI3K/AKT/ mTOR pathway emerges as a potential therapeutic target in cancer, as well as gynecological malignancy. ${ }^{176,182,183}$ There are many drugs being tested in each part of this pathway: PI3K inhibitors, mTOR inhibitors, AKT inhibitors, and dual inhibitors on $\mathrm{PI} 3 \mathrm{~K} / \mathrm{mTOR}$ or PI3K/AKT. mTOR inhibitors (everolimus and temsirolimus) and PI3K inhibitors (idelalisib, alpelisib and copanlisib) have been FDA-approved to be effective in the advanced cancer treatment, such as breast cancer, renal cell carcinoma, and lymphoma. ${ }^{164}$ Despite there are a number of preclinical/clinical data on PI3K/AKT/mTOR pathway inhibitors, currently there is no FDA-approved indication in gynecological cancers.

mTOR inhibitors

The most tested drugs in the PI3K/AKT/mTOR pathway are those blocking mTOR activity. Temsirolimus, everolimus, and ridaforolimus are the most-studied mTOR inhibitors in gynecological cancers. The results of completed clinical trials (phase II) investigating the safety and efficacy of them in gynecological cancers are summarized in Table 7.

Consistent with preclinical findings, ${ }^{171,184-186}$ initial clinical trials demonstrated promising activities of mTOR inhibitors in EC. Temsirolimus, an intravenous mTORC1 inhibitor ( $25 \mathrm{mg}$ weekly), showed efficacy as monotherapy for advanced and recurrent EC with ORRs of $22-25 \%{ }^{187-189}$ Ridaforolimus is another intravenous mTORC1 inhibitor, administrated at a dose of $12.5 \mathrm{mg}$ daily for 5 consecutive days every 2 weeks, showing a modest therapeutic efficacy as a single agent. ${ }^{190}$ A phase II trial studied the efficacy and tolerability of ridaforolimus in recurrent and advanced EC with an ORR of $8.8 \%$ and a SD of $52.9 \% .{ }^{191}$ Everolimus, an oral mTORC1 inhibitor (10 mg daily), was evaluated in a phase II study (NCT00087685) for the treatment of patients with recurrent or persistent EC, showing an ORR of $0 \%$ and a SD of $43 \%{ }^{192}$ However, everolimus was reported to have the best effects in recurrent EC when combined with hormonal therapy (e.g., letrozole, an aromatase inhibitor), showing ORRs of
29-32\%. ${ }^{193,194}$ Given that mTOR inhibitors are cytostatic cell cycle agents with a benefit mainly in terms of disease stabilization rather than disease response (tumor shrinkage), we found only modest effects of mTOR inhibitors as monotherapy in OC and CC based on current clinical evidence. ${ }^{195}$ Reasons to these disappointing results might be: (1) one pathway blockade is insufficient; combined therapies are needed; (2) analogs of rapamycin selectively inhibit mTORC1; the other mTOR complex, mTORC2, is a positive regulator of AKT; (3) predictive biomarkers are required to identify population who can get most benefit from this pathway blockade. Considering the evidence from preclinical studies showing promising activity of mTOR inhibitors in combination with chemotherapy, a number of clinical trials assessed the efficacy of the addition of mTOR to cytotoxic drugs, as well as novel combination of different targeted therapies. A Phase II trial (NCT01031381), evaluating everolimus plus bevacizumab in recurrent OC, reported that $28 \%$ patients were progression-free at 6 months. Patients with both platinumsensitive and -resistant disease showed response. Overall, the regimen was well-tolerated. ${ }^{196} \mathrm{~A}$ randomized Phase II trial (NCT00977574) compared the efficacy of temsirolimus in combination with chemotherapy (carboplatin and paclitaxel) to bevacizumab plus chemotherapy in advanced or recurrent EC. Patients treated by temsirolimus plus chemotherapy had an ORR of $55.3 \%$, and a median OS of 25 months. However, the results reported no improvement in comparison to bevacizumab plus chemotherapy. ${ }^{197}$ A phase I trial (NCT02193633) investigated the efficacy of vistusertib (a dual mTORC1/mTORC2 inhibitor) in combination with paclitaxel in OC, showing an ORR of $52 \%$ and a median PFS of 5.8 months. ${ }^{198}$ Currently, no specific predictive biomarker has been recognized. Tumors with PI3K or PTEN mutations did not necessarily respond to mTOR inhibitors. ${ }^{199}$ Common treatment-related AEs of mTOR inhibitors include stomatitis, mucositis, pneumonitis, rash, fatigue, anemia, diarrhea, nausea, vomiting, hyperglycemia, and immunosuppression.

\section{AKT inhibitors}

GSK2141795 and MK2206 are inhibitors targeting AKT, acting upstream of mTOR. ${ }^{200,201}$ A phase II trial tested dual inhibition of PI3K and Ras signaling by combining the AKT inhibitor (GSK2141795, $50 \mathrm{mg}$ orally daily) and the MEK inhibitor (trametinib, $1.5 \mathrm{mg}$ orally daily) in recurrent $\mathrm{CC}$, with $\mathrm{AEs}$ including gastrointestinal events, fatigue, and rash. One patient had an unconfirmed partial response, with an ORR of $7.1 \%$. Eight patients $(57.1 \%)$ had stable disease. ${ }^{202}$ However, the combination of trametinib and GSK2141795 was shown to have high levels of toxicity in EC at this dose. And the preliminary efficacy is disappointing in another phase II trial (NCT01935973). ${ }^{203,204}$ Moreover, a two-arm, PIK3CA mutation stratified phase II trial (NCT01307631) in recurrent EC demonstrated limited single-agent activity of MK2206 (200 mg orally weekly) in both PIK3CA mutant and wild-type populations. ${ }^{205}$ Afuresertib, another AKT inhibitor, combined with chemotherapy showed an acceptable safety profile in patients with platinum-resistance $O C$ in a phase I study. ${ }^{206}$ A phase II trial of afuresertib plus weekly paclitaxel in platinum-resistance OC (NCT04374630, PROFECTA-II) is under way.

\section{PI3K inhibitors}

BKM120 (buparlisib) is an oral pure PI3K inhibitor. It was shown to have antitumor activity in preclinical and early trials. ${ }^{207,208}$ However, a phase II trial (NCT01397877) demonstrated that the BKM120 (100 mg orally daily) was associated with a minimal antitumor activity as monotherapy in advanced or recurrent EC. ${ }^{209}$ Another oral PI3K inhibitor, pilaralisib (600-mg capsules or 400-mg tablets daily), also had minimal success in a phase II trial in advanced or recurrent EC. ${ }^{210}$ PF-04691502 and gedatolisib (PF05212384) are potent, dual PI3K/mTOR inhibitors. ${ }^{211} \mathrm{~A}$ randomized phase II non-comparative trial (NCT01420081) was 
Table 7. Completed phase II trials of PI3K/AKT/mTOR pathway inhibitors in gynecological cancers

\begin{tabular}{|c|c|c|c|c|c|c|c|c|c|}
\hline ID & Cancer/condition & No. & Intervention & ORR (\%) & CBR (\%) & mPFS (mon.) & mOS (mon.) & SAEs (\%) & Refs \\
\hline NCT001460979 & EC/advanced & 22 & Temsirolimus & 10 & 35 & 3.0 & 21.3 & - & 415 \\
\hline AGO-GYN8 & OC/advanced & 22 & & 4.8 & 38.1 & 3.4 & 21.9 & & \\
\hline NCT00429793 & OC/recurrent & 54 & Temsirolimus & 9.3 & - & 3.1 & 11.6 & 9.26 & 416 \\
\hline NCIC IND 160 & $\mathrm{EC} /$ recurrent or metastatic & 23 & Temsirolimus & 26 & 89 & - & - & - & 88 \\
\hline NCT00723255 & $\mathrm{EC} /$ recurrent & 53 & $\begin{array}{l}\text { Temsirolimus }+ \\
\text { bevacizumab }\end{array}$ & 24.5 & 40 & 5.6 & 16.9 & 63.27 & 417 \\
\hline \multirow[t]{2}{*}{ NCT00729686 } & $\mathrm{EC} /$ advanced or recurrent & 71 & (1) Temsirolimus & 22 & 52.4 & 4.9 & 10.8 & 36 & 187 \\
\hline & & & $\begin{array}{l}\text { (2) Temsirolimus + } \\
\text { hormone therapy }\end{array}$ & 14.3 & - & & & 61.9 & \\
\hline \multirow[t]{2}{*}{$\begin{array}{l}\text { NCT00072176 } \\
\text { NCIC CTG }\end{array}$} & $\begin{array}{l}\text { EC/locally advanced, } \\
\text { recurrent, or metastatic }\end{array}$ & 60 & $\begin{array}{l}\text { (1) Temsirolimus + } \\
\text { hormone therapy }\end{array}$ & 14 & 89 & 7.33 & - & 33.33 & 418 \\
\hline & & & $\begin{array}{l}\text { 2) Temsirolimus + } \\
\text { chemotherapy }\end{array}$ & 4 & 50 & 3.25 & & 33.33 & \\
\hline \multirow{3}{*}{$\begin{array}{l}\text { NCT00977574 GOG- } \\
86 \mathrm{P}\end{array}$} & $\mathrm{EC} /$ stage III-IV or recurrent & 349 & (1) Bevacizumab + PC & 59.5 & - & - & 34 & 42.8 & 197 \\
\hline & & & (2) Temsirolimus + PC & 55.3 & & & 25 & 50.4 & \\
\hline & & & (3) Bevacizumab + IC & 52.9 & & & 25.2 & 46.5 & \\
\hline $\begin{array}{l}\text { NCT01026792 } \\
\text { NCIC IND199 }\end{array}$ & $\mathrm{CC} /$ advanced or metastatic & 38 & Temsirolimus & 3 & 60.6 & 3.52 & - & 40.5 & 419 \\
\hline NCT00087685 & $\begin{array}{l}\mathrm{EC} / \text { progressive or } \\
\text { recurrent }\end{array}$ & 35 & Everolimus & 21 & 45.1 & - & - & - & 192 \\
\hline NCT01068249 & $\mathrm{EC} /$ recurrent & 38 & Everolimus + letrozole & 32 & 40 & 3 & 14 & 31.6 & 194 \\
\hline NCT01797523 & $\mathrm{EC} /$ recurrent & 58 & $\begin{array}{l}\text { Everolimus + letrozole }+ \\
\text { metformin }\end{array}$ & 29 & 66.7 & - & - & - & 193 \\
\hline NCT02283658 & $\mathrm{OC} / \mathrm{ER}+$, recurrent & 20 & Everolimus + letrozole & 16 & 37 & 3.9 & 13 & 63 & 420 \\
\hline \multirow[t]{2}{*}{ NCT00739830 } & $\mathrm{EC} /$ stage III-IV & 130 & $\begin{array}{l}\text { (1) Hormone or } \\
\text { chemotherapy }\end{array}$ & 4 & 17 & 1.9 & - & 34 & 421 \\
\hline & & & (2) Ridaforolimus & 0 & 35 & 3.6 & & 57 & \\
\hline NCT00122343 & $\mathrm{EC} /$ recurrent & 45 & Ridaforolimus & 11 & 19 & - & - & 33 & 422 \\
\hline NCT00770185 & $\mathrm{EC} /$ recurrent & 35 & Ridaforolimus & 8.8 & 62 & - & - & 37.1 & 423 \\
\hline- & $\mathrm{EC} /$ progressive & 45 & Ridaforolimus & 7.4 & 33 & - & - & 35.6 & 424 \\
\hline NCT01935973 & $\mathrm{EC} /$ recurrent or persistent & 26 & GSK2141795 + trametinib & 8.3 & - & - & - & 61 & 203 \\
\hline NCT02538627 & $\mathrm{CC} /$ persistent or recurrent & 35 & GSK2141795 + trametinib & 7.1 & 44 & 3.6 & 14.8 & 57 & 202 \\
\hline NCT01307631 & $\mathrm{EC} /$ recurrent & 37 & MK2206 & 5.5 & 33 & - & 8 & 37.84 & 205 \\
\hline $\begin{array}{l}\text { NCT01397877 } \\
\text { ENDOPIK }\end{array}$ & $\mathrm{EC} /$ advanced or recurrent & 40 & BKM120 & 0 & 60 & 4.5 & & 21 & 209 \\
\hline NCT02193633 & OC/HGSOC & 27 & $\begin{array}{l}\text { Vistusertib }+ \\
\text { chemotherapy }\end{array}$ & 52 & 78 & 5.8 & - & - & 198 \\
\hline NCT01587040 & $\mathrm{EC} /$ advanced or recurrent & 67 & Pilaralisib & 6 & 13.4 & - & - & 52.9 & 10 \\
\hline NCT01420081 & $\mathrm{EC} /$ recurrent & 40 & Gedatolisib & 16 & 5 & 3.6 & - & & 212 \\
\hline
\end{tabular}

$C B R$ clinical benefit rate $=$ complete response + partial response + stable disease, $E R+$ estrogen receptor positive

conducted in patients with recurrent EC following platinumcontaining chemotherapy. Clinical benefit response criteria were only met in the gedatolisib/stathmin-low arm. ${ }^{212}$ Common treatment-related AEs include nausea, mucositis, decreased appetite, diarrhea, fatigue, vomiting, rash, and stomatitis.

In summary, the role of the PI3K/AKT/mTOR pathway inhibitors in gynecological cancers is not yet clear. The reasons for the unsatisfactory results may be related to the feedback loops and compensatory activation of Ras pathway. Even though the presented clinical results are controversial, ${ }^{213}$ there are amount of preclinical studies and clinical trials in progress, mainly combining $\mathrm{PI} 3 \mathrm{~K}$ signaling blockade with other therapies or different targeted agents. ${ }^{214}$ For example, a randomized phase II trial (NCT02397083) is designed to study how everolimus works with the levonorgestrel-releasing intrauterine system for earlystage EC. Another phase II trial (NCT03008408) is to learn if the combination of everolimus, letrozole, and ribociclib (a CDK4/6 inhibitor) can help to control recurrent or progressive EC. Dual mTORC inhibition continues to be assessed in advanced or recurrent OC (NCT03648489). Furthermore, for the future of this pathway targeted therapy, studies of predictive biomarkers might be very helpful and important.

Human epidermal growth factor receptor-targeted inhibitors Human epidermal growth factor receptors (HERs), also known as erythroblastic leukemia viral oncogene (erbB) family, include HER1 (Erb1, EGFR), HER2 (Erb2), HER3 (Erb3), and HER4 (Erb4). ${ }^{215}$ Structural features of HER proteins include extracellular ligandbinding domain, transmembrane domain, and intracellular protein tyrosine kinase domain. ${ }^{216}$ When ligands bind to the extracellular domain, HERs form homodimers or heterodimers with other members of the family. ${ }^{217}$ As an exception, HER2 does not bind any ligand, but it has the most favorable kinase activity. HER3 lacks tyrosine kinase activity. ${ }^{218}$ Dimerization of ligand-activated HERs 


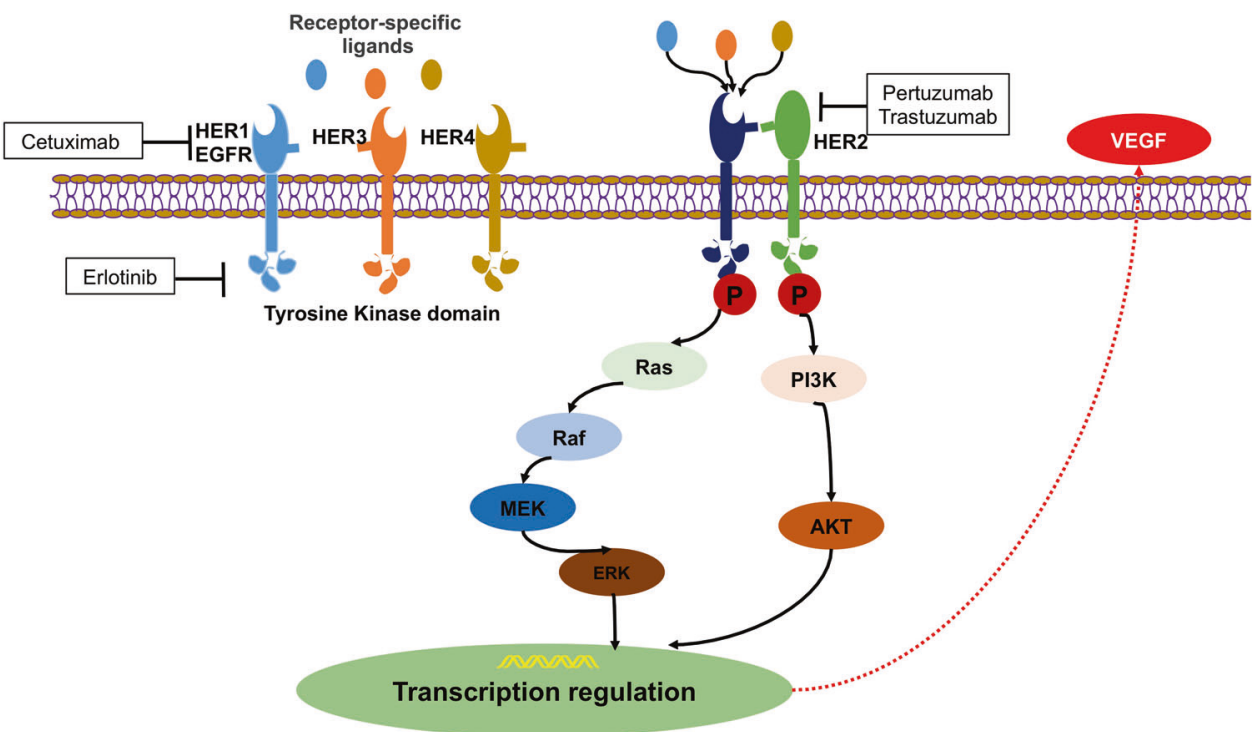

Fig. 3 The HER signal transduction pathway and therapeutic interventions

initiates a cascade of downstream signaling, such as PI3K/AKT/ mTOR, Ras/Raf/MAPK (the mitogen-activated protein kinase pathway), and JAK/ STAT (the signal transducer and activation of the transcription pathway), which regulate from cell division to death, motility to adhesion (Fig. 3). ${ }^{216}$ Overexpression of EGFR and HER2 protein and amplification of HER2 oncogene play an important role in carcinogenesis, associated with breast, lung, gastric, ovarian, endometrial, and bladder cancer. ${ }^{219-222}$ HER2 is also related to increased recurrence and poor prognosis in some cancers. ${ }^{223}$ Thus, EGFR and HER2 are promising targets for treatment of cancer. $224-227$

HER-targeted drugs include monoclonal antibodies and smallmolecule inhibitors. Monoclonal antibodies against the extracellular domain of the HER receptor include cetuximab, nimotuzumab, trastuzumab, pertuzumab, and ado-trastuzumab emtansine (T-DM1). ${ }^{227-229}$ Cetuximab and nimotuzumab bind to the extracellular domain of the EGFR. Trastuzumab obstructs HER2 homodimerization. HER2 overexpression is required for trastuzumab to be effective. ${ }^{230}$ Pertuzumab inhibits HER2 heterodimerization and does not require HER2 overexpression to be effective. ${ }^{231}$ T-DM1 is trastuzumab conjugated to emtansine (a microtubule inhibitor), which inhibits microtubule assembly in the cytoplasm and thus leads to cell death. ${ }^{232}$ Small-molecule inhibitors are TKIs including gefitinib, erlotinib, lapatinib, and afatinib against intracellular kinase domain to prevent signaling. ${ }^{233}$ Among them, gefitinib and erlotinib are inhibitors selective for EGFR. ${ }^{234,235}$ Lapatinib and afatinib inhibit both EGFR and HER2. ${ }^{236}$ Most of them have been approved by FDA as targeted therapies for certain advanced or recurrent cancers with selected biomarkers, such as breast cancer, colorectal cancer and non-small cell lung cancer (NSCLC).

As for gynecological cancers, HER2 is an important oncogene in high grade and stage EC, especially in uterine serous carcinoma. ${ }^{237,238}$ In OC, the rate of HER2 overexpression is highly variable (ranging from $2 \%$ to $66 \%$ ), and the rate of EGFR overexpression is $30-70 \% .^{239,240}$ In CC, the rate of EGFR overexpression ranges from $6 \%$ to $90 \%{ }^{241,242}$ However, unlike in NSCLC, ${ }^{243}$ the clinical significance of EGFR/HER2 gene amplification or protein overexpression and the efficacy of HER-targeted therapy are still controversial in gynecological cancers (Table 8).

\section{Cetuximab}

Cetuximab was demonstrated to have no additional benefit beyond chemotherapy in several phase II trials for CC. ${ }^{244,245}$
Moreover, in a phase II trial, the combination of cetuximab and topotecan induced a high rate of serious adverse reactions in the treatment of advanced CC. ${ }^{246}$ Another randomized phase II trial, MITO CERV-2 (NCT00997009), studied the efficacy of cetuximab plus carboplatin and paclitaxel in advanced or recurrent CC, showing no significant improvement in either the median PFS or the median OS. ${ }^{247}$ For OC, a phase II trial (NCT00086892) demonstrated modest activity of cetuximab in combination with carboplatin in patients with platinum-sensitive recurrent $O C$ with an ORR of $32.1 \%$ and an increased incidence of hypersensitivity reactions. ${ }^{248}$ There is limited information about the clinical efficacy of cetuximab in EC.

\section{Trastuzumab}

Trastuzumab treatment revealed no responses in a phase II trial with HER2-positive EC (NCT00006089). ${ }^{249}$ However, another randomized phase II trial (NCT01367002) of paclitaxel and carboplatin with or without trastuzumab in primary stage III or IV or recurrent HER2-positive uterine serous carcinomas showed an improvement in the median PFS in the trastuzumab combination arm (4.6 months longer, $P=0.005)$. In the population with primary advanced-stage disease, the median PFS was 17.9 months in the trastuzumab combination arm versus 9.3 months in the chemotherapy alone arm. In the population with recurrent disease, the median PFS was 9.2 versus 6 months, respectively. ${ }^{250}$ For patients with HER2 overexpression OC, trastuzumab showed modest activity with an ORR of $7.3 \%$ in a phase II trial. ${ }^{239}$ A clinical study in china demonstrated that the combination of abraxane and trastuzumab might have promising efficacy and adverse reaction in the treatment of recurrent $O C$, showing a control rate of $86.4 \% .^{251}$ However, there is limited information about the clinical efficacy of trastuzumab for CC.

\section{Pertuzumab}

A randomized phase II trial (NCT00096993) of chemotherapy (gemcitabine) with or without pertuzumab in patients with platinum-resistant OC demonstrated an increased ORR in the pertuzumab combination arm. ${ }^{252}$ Furthermore, a phase III trial, PENELOPE (NCT01684878), evaluated the addition of pertuzumab to chemotherapy in patients with platinum-resistant OC with low tumor HER3 mRNA expression. However, the differences in the median PFS and OS were not statistically significant. ${ }^{253,254}$ In unselected patients with platinum-sensitive recurrent $O C$, a phase II trial (NCT02004093) showed that the addition of pertuzumab to 


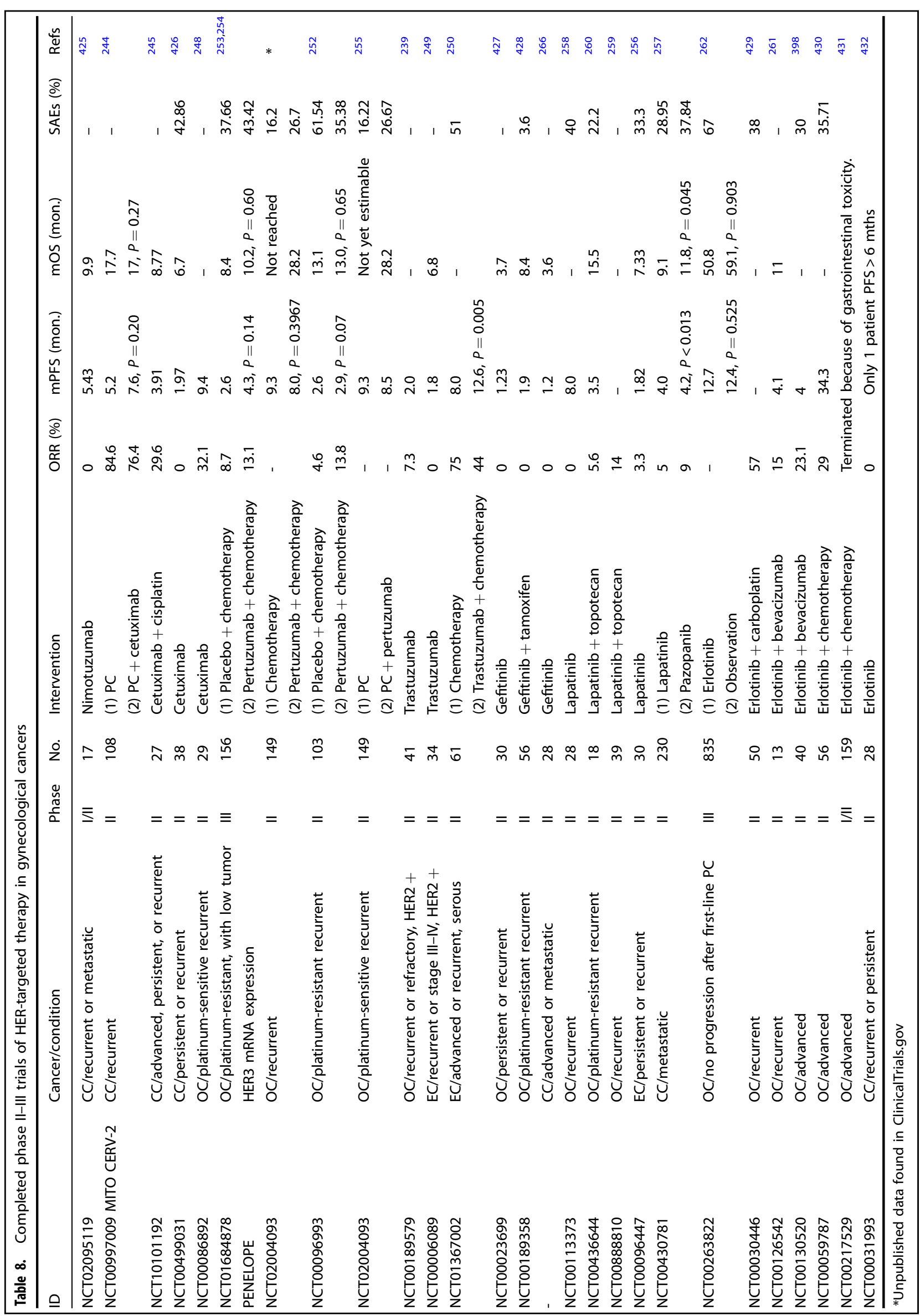


16

carboplatin-based chemotherapy did not substantially prolong PFS. ${ }^{25}$ Also, there is limited information about the clinical efficacy of pertuzumab for EC and CC.

TKIs

In clinical trials of small-molecule inhibitors, a phase II trial (NCT00096447) tested the efficacy of lapatinib and explored biological characteristics in persistent or recurrent EC. ${ }^{256}$ The analysis demonstrated that lapatinib had limited activity in unselected cases in EC, as well as in OC and CC. ${ }^{257-260} \mathrm{~A}$ phase II trial assessed the activity and tolerability of the combination of bevacizumab and erlotinib in recurrent OC with an ORR of $15 \%{ }^{261}$ Furthermore, a phase III trial (NCT00263822), evaluating the efficacy of maintenance erlotinib in OC patients after first-line chemotherapy, showed no improvement in PFS or OS. ${ }^{262}$ Moreover, this study failed to show a consistent correlation between EGFR mutational status/protein expression and clinical outcomes. For CC, a phase II trial evaluated the efficacy of erlotinib combined with chemoradiation in treating patients with locally advanced CC, showing a promising activity with a complete response of $94.4 \%{ }^{263}$ Other HER-targeted TKIs (e.g., gefitinib, canertinib, and vandetanib) showed minimal clinical activities in gynecological cancers in current clinical trials. ${ }^{242,264-267}$

Even though the present clinical evidences are not very satisfying, HER-targeted therapies continue to be investigated in gynecological cancers for their potent value for biomarkerselected patients (e.g., NCT01388621, NCT01367002, NCT02039791, NCT00292955, NCT03469531, NCT00317772, NCT01953926). Furthermore, preclinical data suggested the potential of novel combination strategies involving HER-targeted therapy, which are also investigated in ongoing clinical trials. $227,268-270$

\section{Other molecular targeted therapies}

Ras/Raf/MEK. In the Ras/Raf/MEK signaling pathway, Ras activation is the first process in activation of the mitogen-activated protein kinases (MAPKs) cascade. ${ }^{271}$ Upon Ras activation, Raf is recruited to the cell membrane where subsequent changes in Raf phosphorylation status result in activating MEK kinases (MEK1 and MEK2). ${ }^{272}$ MEK1 and MEK2 furtherly trigger Erk1 and Erk2. Finally, Erks regulate the activity of several transcription factors that induce the expression of multiple genes required for important cell activities (Fig. 4). ${ }^{273}$ The dysregulation of this pathway exists in many human tumors, making it an attractive antitumor target. Intensive preclinical researches have led to identifying Raf kinase inhibitors, as well as inhibitors of its downstream effector MEK
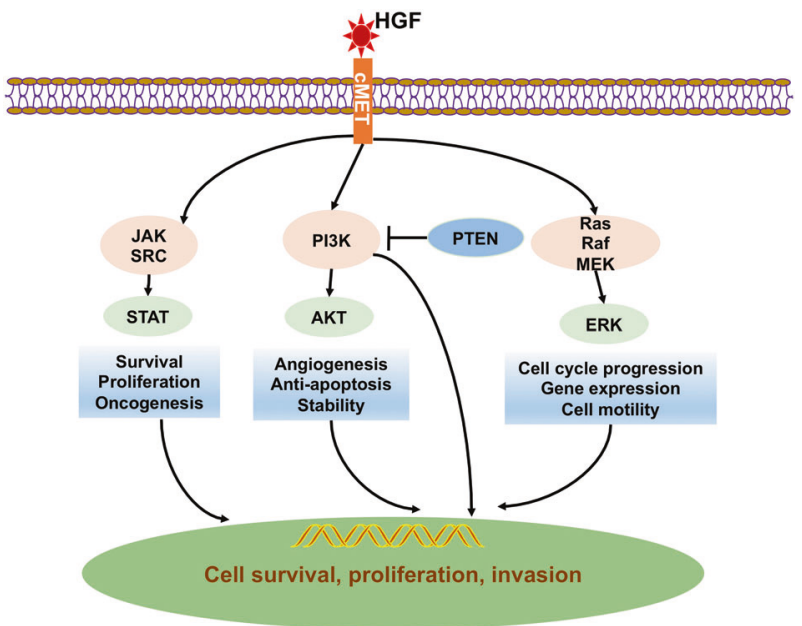

Fig. 4 The HGF/c-MET signal transduction pathway and therapeutic interventions kinase. $^{274-276}$ The results of the completed phase II trials, which evaluated the efficacy of the Ras/Raf/MEK pathway inhibitors in treating gynecological cancers, are summarized in Table 9.

Lonafarnib is an orally protein farnesyltransferase inhibitor for $\mathrm{H}$-ras, K-ras-4B, and N-ras. ${ }^{277}$ The addition of lonafarnib into firstline chemotherapy was investigated in a phase II trial (NCT00281515), and no effect was observed on prolonging PFS or $O S$ in advanced $\mathrm{OC}^{278}$ Sorafenib is a non-selective oral multikinase inhibitor with effects on angiogenesis through inhibition of the VEGF receptor. ${ }^{279,280}$ In addition, the antitumor effect of sorafenib is thought to be mediated through its inhibition of the Ras/Raf/MEK pathway, which is also frequently activated in advanced $\mathrm{OC}^{281,282}$ It has been evaluated in more than 100 clinical trials in different cancer types, especially in large phase III studies in renal and liver cancers. ${ }^{283-285}$ It has been approved by the FDA for the treatment of renal, thyroid, and hepatocellular carcinoma. In OC, sorafenib showed antitumor activity in xenograft models and clinical studies. ${ }^{286,287}$ However, the results from a phase II trial (NCT00390611) in first-line treatment and maintenance therapy of OC showed no effect on prolonging PFS in sorafenib combination arm versus chemotherapy alone. ${ }^{288}$ The similar results were reported in patients with OC in complete remission (NCT00791778). ${ }^{289}$ On the other hand, a phase II trial (NCT01047891) demonstrated that sorafenib combined with topotecan as maintenance therapy significantly improved in the median PFS (2.3 months longer, $P=0.0018)$ and OS (7.1 months longer, $P=0.017$ ) in patients with platinum-resistant recurrent $\mathrm{OC}^{290}$ In patients with platinum-sensitive recurrent OC, sorafenib in combination with carboplatin and paclitaxel was reported to show promising activity with an ORR of $61 \% .{ }^{291}$ Sorafenib was tested in early-phase clinical trial for CC patients receiving concurrent chemoradiation. ${ }^{292}$ However, it ended with early closure.

Selumetinib is an oral selective inhibitor of MEK1 and MEK2. It has shown activity against several advanced cancers. ${ }^{293,294}$ Since mutational alterations were found in the MAPK pathway in OC and EC, selumetinib-related clinical trials were conducted in gynecological cancers. ${ }^{281,295,296}$ For EC, selumetinib is well-tolerated in patients with recurrent or persistent disease, but with limited single-agent activity with an ORR of $6 \%$ (NCT01011933). ${ }^{297}$ A phase II trial (NCT00551070) demonstrated the potential activity of selumetinib in the treatment of recurrent low-grade OC with an ORR of $15 \%$. It was suggested that inhibitors of the MAPK pathway should be further investigated in OC patients. ${ }^{298}$ Subsequently, an ongoing randomized phase II/III trial (NCT02101788) continues to study how well trametinib (another MEK inhibitor) works and compares it to the standard treatment in treating patients with low-grade OC. Trametinib, combined with GSK2141795 (an AKT inhibitor), has previously been tested in phase I and II studies. ${ }^{299}$ However, phase II clinical trials assessing this combination in EC (NCT01935973) or OC (NCT02538627) showed no clinical benefit. $^{202,203}$ A phase II trial tested the combination of trametinib and GSK2141795 in recurrent CC with no confirmed response and a SD of $57 \% .^{202}$

In summary, while a powerful preclinical rationale suggests that inhibition of Ras/Raf/MEK signaling has promising potent as an antitumor targeted therapy, the clinical efficacy of this strategy in gynecological cancers is currently limited.

JAK/STAT. The janus kinase/signal transducer and activator of the tran-ions (JAK/STAT) pathway has been proved to mediate the action of cytokines, interferons and growth factors, and their control of gene expression. ${ }^{300}$ Activation of the JAK/STAT pathway and overexpression of STAT have been seen in many malignancies such as colorectal and breast cancers. ${ }^{301,302}$ Therefore, the JAK/ STAT pathway is being focused as a potential target in cancer therapies. Ruxolitinib is an FDA-approved drug of JAK for treatment of patients with polycythemia vera. ${ }^{303}$ Preclinical 
Table 9. Phase II trials (with results) of molecular targets in gynecological cancers

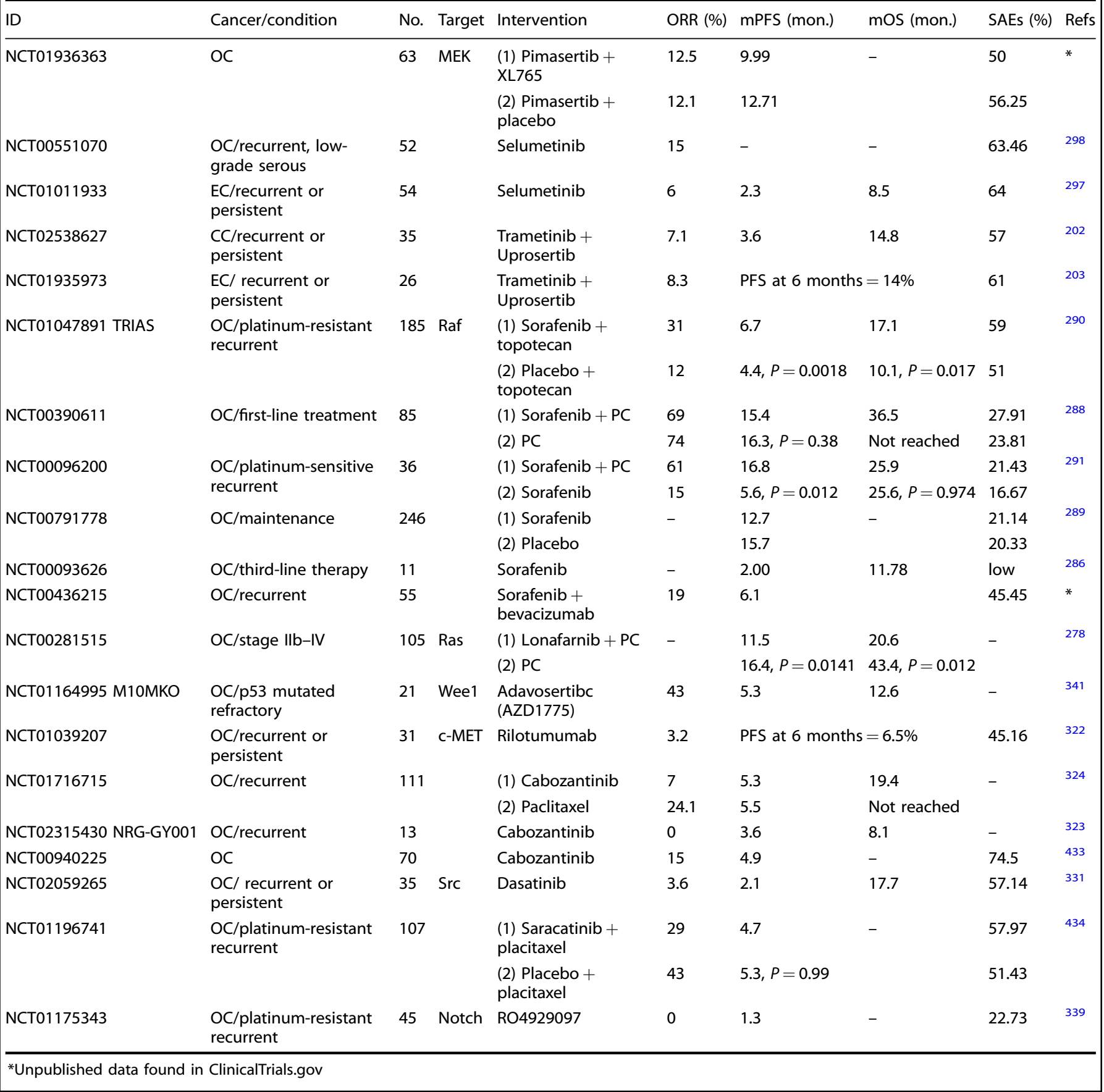

studies demonstrated that ruxolitinib reduced OC cell viability. ${ }^{304,305}$ It enhanced the sensitivity of OC cells to other anticancer agents, and suppressed ovarian tumor growth in mice. ${ }^{306,307}$ These results supported the clinical investigation of ruxolitinib in OC patients. A phase I/II trial (NCT02713386) is trying to explore the effect of ruxolitinib phosphate when given together with paclitaxel and carboplatin in treating patients with stage III-IV OC

HGF/c-MET. Tyrosine kinase receptor c-MET (cellular-mesenchymal to epithelial transition factor) is activated by hepatocyte growth factor (HGF) and it can trigger important cellular processes. ${ }^{308}$ Upon binding by HGF, MET is dimerized and activates cellular processes through the Ras/Raf/MEK and PI3K/AKT/mTOR pathways (Fig. 4). ${ }^{309,310}$ In a limited number of tumors, MET genetic lesions or mutations lead to the constitutive activation of MET. ${ }^{311,312}$ However, in a majority of malignancies, aberrant MET signaling derives from the upregulation of HGF transcription, leading to receptor and ligand overexpression. ${ }^{313-315}$

Since the publications of pioneer studies, the HGF/c-MET system has gained growing attention with its role in the pathogenesis of gynecological cancers. ${ }^{316,317}$ In a study analyzing 1115 advanced cancer patients, MET amplification was detected in $2.6 \%$ patients with solid tumors. ${ }^{318}$ But in OC, MET overexpression was detected in more than $20 \%$ (range from $22 \%$ to $41 \%$ ) ovarian clear cell adenocarcinomas. ${ }^{319,320}$ And increased expression of HGF and 
18

c-Met signaling is associated with a poor prognosis of EC patients. ${ }^{321}$ Therefore, targeting the interaction of c-MET and HGF would be beneficial in treating gynecological cancers. Despite there are massive preclinical data on the HGF/c-MET axis, currently there is no FDA-approved indication of this targeted therapy in cancers.

The most tested drugs in HGF/c-MET axis are those blocking cMET activity. Rilotumumab and cabozantinib are the most-studied c-MET inhibitors in gynecological cancers. The results of the completed clinical trials (phase II) investigating the safety and efficacy of them in gynecological cancers are summarized in Table 9. A phase II trial (NCT01039207) evaluated the rilotumumab in the treatment of persistent or recurrent OC. Only $1 / 31$ achieved objective response, and only two patients got 6-month PFS. ${ }^{322} \mathrm{~A}$ phase II trial (NCT02315430) evaluated cabozantinib in treating patient with recurrent clear cell OC with no response. ${ }^{323}$ Another phase II trial (NCT01716715) compared cabozantinib versus weekly paclitaxel in treatment of persistent OC, with even worse OS and ORR in cabozantinib arm. ${ }^{324}$ These results do not warrant further evaluation of rilotumumab or cabozantinib as a single agent in targeted therapy of OC. There is currently limited information of the clinical efficacy of these agents in EC and CC.

Src. Sarcoma proto-oncogene tyrosine kinase (Src) is a downstream component of many growth factor receptors, such as VEGFR, EGFR, and c-MET. ${ }^{325}$ Src is thought to increase chemotherapy resistance through activating Ras and AKT. ${ }^{326}$ Preclinical studies showed that inhibiting Src resulted in enhancing apoptosis caused by cytotoxic drugs, such as paclitaxel, carboplatin, and gemcitabine. ${ }^{327,328}$ Src has been found to be overexpressed in gynecological cancers and promote resistance against chemotherapy. ${ }^{328,329}$ Dasatinib and saracatinib are the most-studied highly selective Src inhibitors in gynecological cancers. ${ }^{330}$ In a phase II trial (NCT01196741), it was reported that saracatinib did not improve activity of weekly paclitaxel in platinum-resistant OC. ${ }^{331}$ Another phase II trial (NCT02059265) showed that dasatinib had minimal activity as a single agent in patients with recurrent $O C^{332}$ Even though no obvious activity has been seen as a single agent, Src inhibitors used in combination with other antitumor agents are promising.

Notch. Notch signaling is a primordial, evolutionarily conserved cell-fate determination pathway that has great relevance to multiple aspects of cancer biology, from cancer stem cell to tumor immunity. ${ }^{333,334}$ Previous studies have shown that the Notch pathway is associated with the epithelial-mesenchymal transition (EMT) processes in OC and CC. ${ }^{335-338}$ Currently, several classes of Notch inhibitors have been developed, mainly composed of gamma-secretase inhibitors (GSIs), siRNA, and monoclonal antibodies against Notch pathways. ${ }^{336}$ RO4929097 is a GSI, which had insufficient activity as a single agent in platinumresistant OC in a phase II clinical trial (NCT01175343). ${ }^{339}$

Cell cycle checkpoints. Wee1 is a kinase controlling G/M and S phase checkpoints via phosphorylation of the cyclin-dependent kinases. Ataxia-telangiectasia-mutated-and-Rad3-related kinase (ATR) plays an important role in the DNA damage response to replication stress, preventing the entry of cells with damaged DNA into mitosis (e.g., when the cancer cells are challenged by chemotherapy). ${ }^{340}$ These functions of Wee1 and ATR make them potential therapeutic targets. The activities of ATR inhibitors (e.g., AZD6738) and Wee1 inhibitors (e.g., AZD1775) have been investigated in early-phase trials in gynecological cancers ${ }^{341}$ (Tables 9 and 14).

Antibody-drug conjugates. Antibody-drug conjugates (ADCs) are complex engineered molecules composed of a monoclonal antibody conjugated to payload (e.g., cytotoxic drugs) via stable linkers. ${ }^{342,343}$ By binding to the antigens on the tumor cell surface, the ADCs release the drug components intracellularly and lead to the death of tumor cell. This site-selective drug delivery can reduce toxicities for patients by limiting the exposure of normal tissues to the cytotoxic drugs. ${ }^{344}$

Mirvetuximab soravtansine is an ADC for treatment of folate receptor a (FRa)-expressing tumors, comprising a humanized $\mathrm{FRa}$ binding monoclonal antibody, a cytotoxic maytansinoid effector molecule DM4, and a cleavable disulfide linker. ${ }^{345-347}$ The FRa mediates the endocytotic uptake of folate, which has a role in amino acid, DNA and RNA metabolism as well as in methylation reactions. ${ }^{348} \mathrm{FRa}$ is overexpressed in several cancers, including ovarian, lung, renal, endometrial, colorectal and breast cancers. ${ }^{349}$ Thus, it is a promising target for ADC design. The FRa expression in tumor is a response-predictive biomarker for patient selection. Preclinical studies showed it to have potent antitumor activities in OC xenografts. ${ }^{350}$ Phase I trials of mirvetuximab soravtansine in OC were conducted. ${ }^{347,351}$ In a population of patients with FRapositive and platinum-resistant $O C$, mirvetuximab soravtansine showed an ORR of $26 \%$ and a median PFS of 4.8 months. ${ }^{352}$ However, the phase III FORWARD I trial (NCT02631876), comparing the safety and efficacy of mirvetuximab soravtansine to chemotherapy in platinum-resistant OC, was terminated because it did not meet prespecified primary endpoints. Another newly registered phase III trial (NCT04209855) is going to compare the efficacy chemotherapy in platinum-resistant $O C$ with a high-level of FRa expression.

Tisotumab vedotin is a monomethyl auristatin $E$ (MMAE) bearing ADC conjugated to an anti-tissue factor (TF) monoclonal antibody via a protease cleavable linker. TF is involved with tumor cell signaling and angiogenesis. Ongoing phase I/II trials GEN701/ GEN702 (NCT02001623, NCT02552121), investigated tisotumab vedotin in solid tumor, including cervical, ovarian, endometrial, and other solid cancers. In the preliminary data released, 11/34 (32.4\%) patients with CC achieved a response. ${ }^{353,354}$

Other ADCs continue to be investigated in a number of ongoing clinical trials (e.g., NCT03748186, NCT03835819, NCT01631552, NCT03657043, NCT03319628, NCT02988817, NCT02751918, NCT02606305, NCT02208375, NCT02996825).

Programmed death protein-1 pathway blockade. Another class of novel alternative therapy in cancer treatment is the immunotherapeutic drug, particularly the agent that inhibits the immune checkpoint. Programmed death protein-1 (PD-1) is an immune checkpoint molecule which is more commonly studied in immunotherapy researches of gynecological cancers. It plays an important role in T-cell coinhibition and exhaustion, and subsequently helps tumor cells evade immune surveillance. ${ }^{355}$ Thus, monoclonal antibodies were developed as a promising cancer therapy targeting at blockading the PD-1 pathway in tumor progression. Although immune checkpoint inhibitors do not target to kill tumor cells directly, they play an antitumor role by enhancing T-cell functions (Fig. 5). The expression of immunosuppressive PD-1 ligands (PD-L1 or PD-L2) on the surface of tumor cells is an important predictive biomarker of response to PD-1 blockade. ${ }^{356,357}$ It is also indicated that mismatch repair-deficient (dMMR) tumors, including dMMR EC, are sensitive to PD-1 blockade. ${ }^{358}$ Anti-PD-1 agents (pembrolizumab and nivolumab) and anti-PD-L1 agents (atezolizumab, avelumab, and durvalumab) were FDA-approved drugs for several kinds of advanced-stage cancers, such as melanoma, NSLC and renal cell carcinoma. ${ }^{359-361}$ In 2017, pembrolizumab was approved by FDA for the treatment of patients with unresectable or metastatic solid tumors with a biomarker referred to as microsatellite instability-high (MSI-H) or dMMR. $^{362}$ These biomarkers are most commonly found in colorectal, gastrointestinal, and endometrial cancers. ${ }^{362-364}$ Successively, pembrolizumab was approved in certain condition of CC and EC (Table 1), basing on findings from two phase II trials 


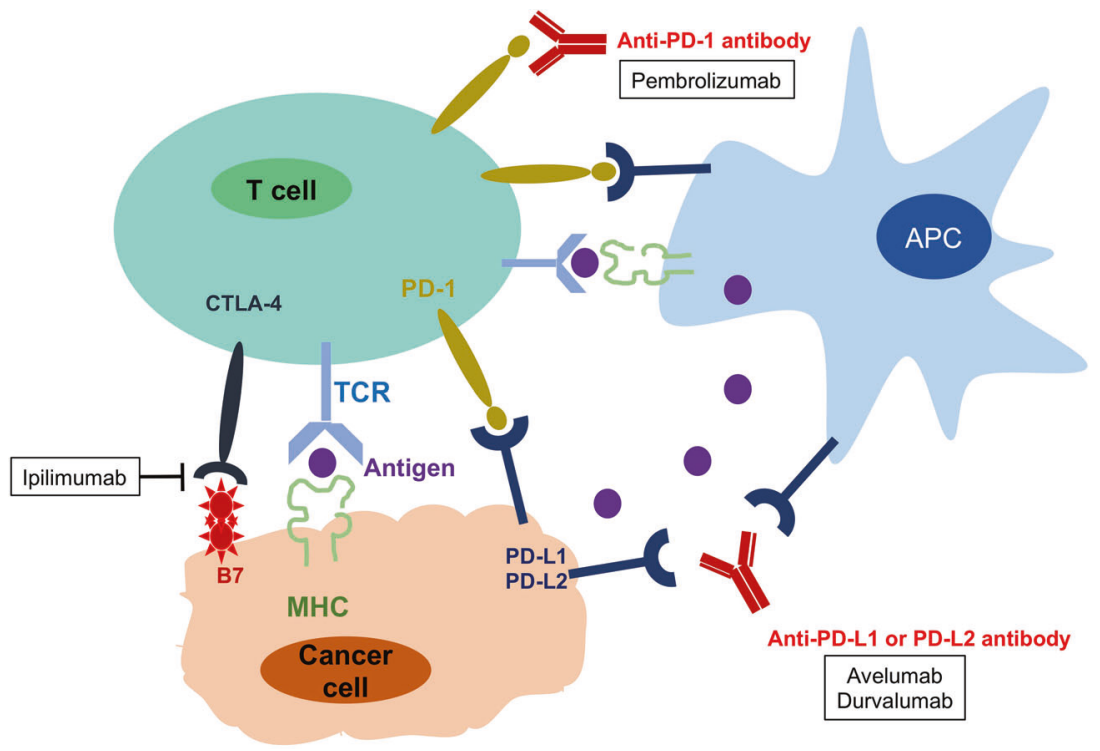

Fig. 5 The immune checkpoint blockades. Antigen presenting cells (APC) take up antigen (Ag) released from tumor cells and present it to T cells. PD-1 receptors inhibit immune responses by engagement of PD-L1 and PD-L2. Therefore, monoclonal antibody blockading the PD-1 pathway results in enhancing antitumor immunity

(KEYNOTE 158 and KEYNOTE 146). ${ }^{365,366}$ The results of the completed phase I/II trials of anti-PD-1/PD-L1 agents for ovarian, cervical, and endometrial cancers are summarized in Table 10. And other ongoing phase II/III trials investigating anti-PD-1/PD-L1 therapy (not in addition to other targeted agents) in gynecological cancers are listed in Tables 11 and 12.

Anti-PD-1 agents. A phase lb KEYNOTE-028 trial (NCT02054806) of pembrolizumab ( $10 \mathrm{mg} / \mathrm{kg}$ intravenously every 2 weeks) as a treatment of PD-L1-positive solid tumors showed that pembrolizumab was associated with a $17 \%$ ORR in CC cohort, a 13\% ORR in EC cohort, and a $11.5 \%$ ORR in OC cohort, respectively. ${ }^{367-369}$ In KEYNOTE 158 trial (NCT02628067), pembrolizumab was investigated in a single cohort of recurrent or metastatic CC, resulting in an ORR of $12.2 \%$. In the population of patients with PD-L1-positive tumors, the ORR was $14.6 \%$. No response was observed in patients with PD-L1-negative tumors. The median OS was 9.4 months in the total population and 11 months in the PD-L1-positive tumor population. ${ }^{365}$ On the ground of this trial, the FDA-approved pembrolizumab for patients with recurrent or metastatic CC with disease progression on or after chemotherapy whose tumors expressed PD-L1, in 2018. As for EC, a phase II study evaluated the clinical efficacy of pembrolizumab in nine patients with recurrent or persistent EC with $\mathrm{dMMR}$, and the results indicated that the ORR was $56 \%$, the 12 -month OS rate was $89 \%$, and the median OS had not been reached. ${ }^{370}$ For EC patients without MSI or PD-L1 expression status, another phase II KEYNOTE 146 trial (NCT02501096) assessed the activity and safety of lenvatinib plus pembrolizumab in patients with biomarker-unselected advanced EC. ${ }^{371}$ Lenvatinib is an oral multikinase inhibitor targeting VEGFR, FGFR, PDGFR, RET, and KIT. ${ }^{372}$ An interim report of KEYNOTE 146 showed this combination of PD-1 blockade and inhibition of angiogenesis (as well as VEGF-mediated immune suppression) was associated with antitumor activity with an ORR of $35.6 \%{ }^{366}$ In September 2019, the FDA granted accelerated approval to the combination of pembrolizumab and lenvatinib for the treatment of patients with advanced EC without MSI-H or dMMR and who have disease progression following prior systemic therapy, but were not candidates for curative surgery or radiation. For patient with recurrent $O C$, single-agent pembrolizumab showed modest activity in a phase II trial (NCT02674061) with an ORR of 7.4-9.9\%. ${ }^{373}$ A phase I/II trial (NCT02657889) demonstrated that niraparib combined with pembrolizumab was tolerable and had promising antitumor activity for platinum-resistant current OC with an ORR of $18 \%$ and a disease control rate of $65 \%{ }^{143}$ Furthermore, a recent study identified two determinants of response to the combination of pembrolizumab and niraparib: the presence of mutational signature 3 as a surrogate of HRD and a positive immune score as a surrogate of interferon-primed, CD8exhausted effector $T$ cells in the tumor microenvironment. Presence of one or both tumor features was associated with significantly prolonged PFS while absence of both was associated with no response. ${ }^{374}$

Nivolumab is another well-known anti-PD-1 drug. As indicated by a phase I/II trial (NCT02488759), nivolumab had a promising activity in metastatic CC with an ORR of $26 \% .{ }^{375}$ However, another phase II trial (NCT02257528) demonstrated that single-agent nivolumab exhibited low antitumor activity in recurrent CC with an ORR of $4 \%$ and a SD of $36 \% .{ }^{376}$ In patients with platinumresistant recurrent $O C$, early-phase trials showed that monotherapy of anti-PD-1 agents had promising activity. ${ }^{377,378}$

Dostarlimab (TSR-042) is an investigational humanized anti-PD-1 monoclonal antibody. It demonstrated robust clinical activity in patients with previously treated recurrent or advanced EC in both MSI-H and MSS subgroups. It is being evaluated in combination of bevacizumab and niraparib in patients with platinum-resistant $O C$ (NCT03574779).

Anti-PD-L1 agents. In a phase la trial (NCT01375842) assessing atezolizumab (10 mg/kg intravenously every 3 weeks) in advanced/recurrent EC, the ORR was $13.3 \%(2 / 15)$ in all populations. Both these two patients were in population with PD-L1 status $>5 \%$ of tumor-infiltrating immune cells $(2 / 5)$. Moreover, a trend for higher PFS and OS was noticed with higher PD-L1 expression. ${ }^{379}$ A phase II trial (NCT02912572) of avelumab (10 mg/ $\mathrm{kg}$ intravenously every 2 weeks) in patients with microsatellite stable (MSS), microsatellite instable (MSI), and POLE-mutated recurrent/persistent EC demonstrated an ORR of $6.25 \%$ in the MSS cohort and an ORR of $27.6 \%$ in the MSI/POLE cohort. ${ }^{380}$ As demonstrated in these clinical outcomes, PD-L1 status, dMMR, $\mathrm{MSI}$, and POLE mutation were predictive biomarkers to identify the EC population who could benefit from PD-1 blockade. However, in patients with recurrent $O C$, a single-agent trial of anti-PD-L1 agents demonstrated only modest efficacy. ${ }^{381}$ 
Table 10. Completed phase I/II trials of anti-PD-1/PD-L1 in gynecological cancers

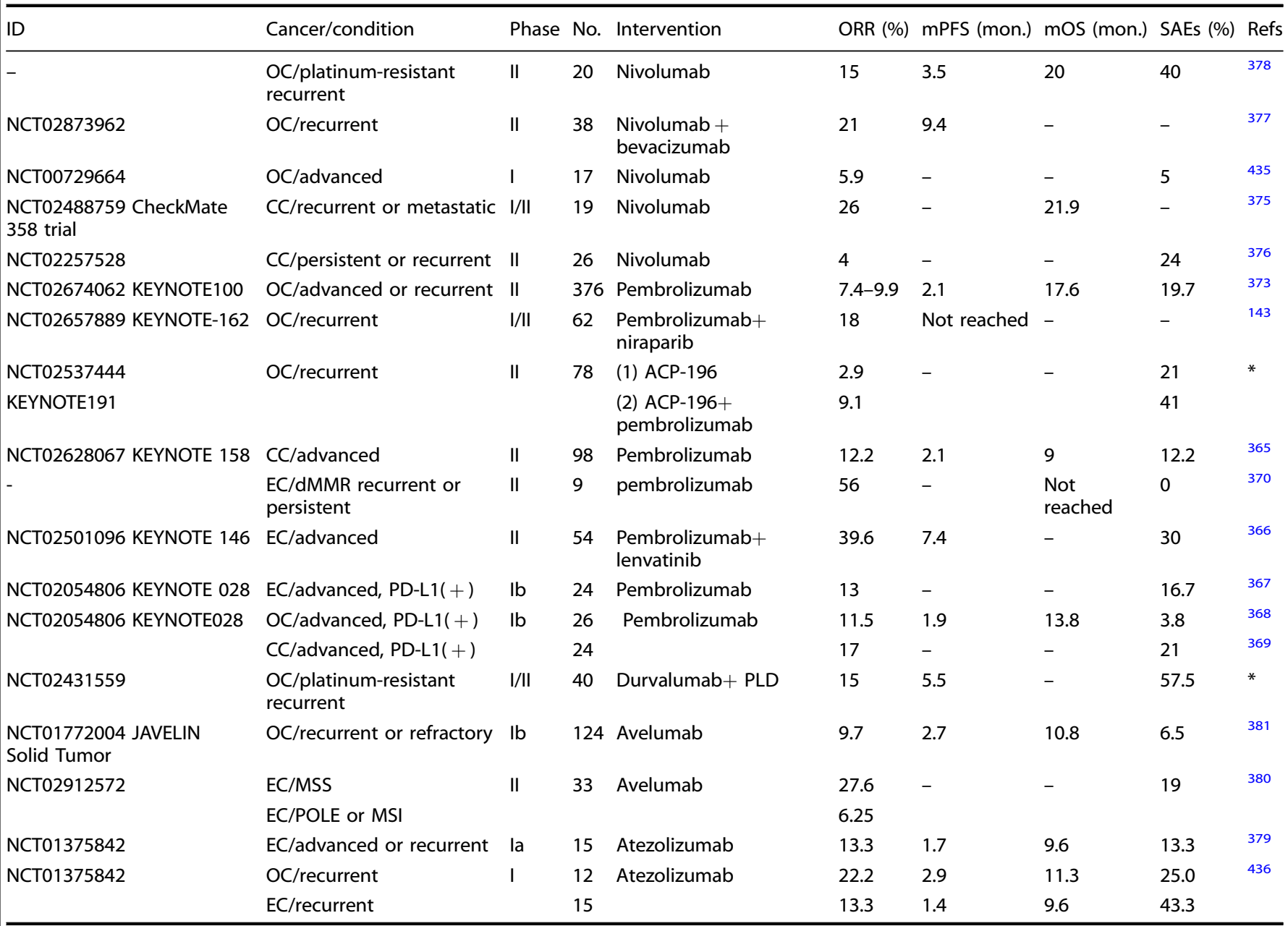

$d M M R$ mismatch repair-deficient, MSS microsatellite stable, MSI microsatellite instable, POLE polymerase- $\varepsilon .{ }^{*}$ Unpublished date found in clinicaltrials.gov

Several clinical trials are further conducted to combine chemotherapy or other targeted therapies with anti-PD-1/PD-L1 agents in treatment of gynecological cancers. A phase II trials showed that the combination of durvalumab $(10 \mathrm{mg} / \mathrm{kg}$ intravenously every 2 weeks) and doxorubicin was associated with an ORR of $15 \%$ in platinum-resistant recurrent OC. A great number of clinical trials have been designed and registered to investigate the efficacy and safety of anti-PD-1/PD-L1 agents combined with other targeted therapy in cancer treatment.

Currently, we find limited results from phase III trials investigating the safety and efficacy of anti-PD-1/PD-L1 agents in gynecological cancers. The reported interim results in $\mathrm{OC}$ are somehow disappointing. JAVELIN Ovarian 100 (NCT02718417), a phase III study of avelumab in combination with chemotherapy treating previously untreated OC patients, was terminated in 2018. The decision of termination was based on the results of a planned interim analysis that showed futility of efficacy. It was further reported that another ongoing phase III study of avelumab for platinum-resistant/refractory recurrent OC, JAVELIN Ovarian 200 (NCT02580058), did not meet prespecified primary endpoints of OS or PFS in patients. As of January 2020, there are dozens of ongoing phase III trials involving anti-PD-1/PD-L1 drugs in gynecological cancers, registered on ClinicalTrials.gov. The ongoing phase III trials are listed in Table 12.

Even though the preliminary results of phase III JAVELIN Ovarian trials are unsatisfying, anti-PD-1/PD-L1 drugs (either used as monotherapy or used in combination with chemotherapy, other immune checkpoint inhibitors, cancer vaccines or other targeted therapies) are still expected to be promising approaches, especially in the treatment of CC and EC. ${ }^{382-384}$

Selective estrogen receptor downregulators. In EC, type I (endometrioid histologies), the most common type, is associated with an excess estrogen exposure in the absence of counteractive effects of progesterone, mostly with expressing estrogen and/or progesterone receptors (ER/PR). ${ }^{385-387}$ Hormonal therapy is an alternative treatment to control metastatic or recurrent disease. . $^{38,389}$ In addition to the conventional progestin therapy, inhibition of estrogen-induced proliferation by anti-estrogenic agents has been evaluated in EC, including selective estrogen receptor modulators (SERMs) or downregulators (SERDs) and aromatase inhibitors. ${ }^{390,391}$

Fulvestrant, the main SERD, has an anti-proliferative effect through down regulation of ER and plays an antitumor role as both hormonal therapy and targeted therapy. Fulvestrant was approved by FDA for the treatment of postmenopausal metastatic ER/PR-positive breast cancer, not yet for gynecological cancers. ${ }^{392}$ A phase II trial (NCT00334295) evaluated the activity and toxicity of fulvestrant, in patients with advanced or recurrent ER/PRpositive EC. ${ }^{393}$ It demonstrated an ORR of $11.4 \%$ in the ITT group, with a median PFS of 2.3 months and a median OS of 13.2 months. However, another phase II trial showed minimal activity of 


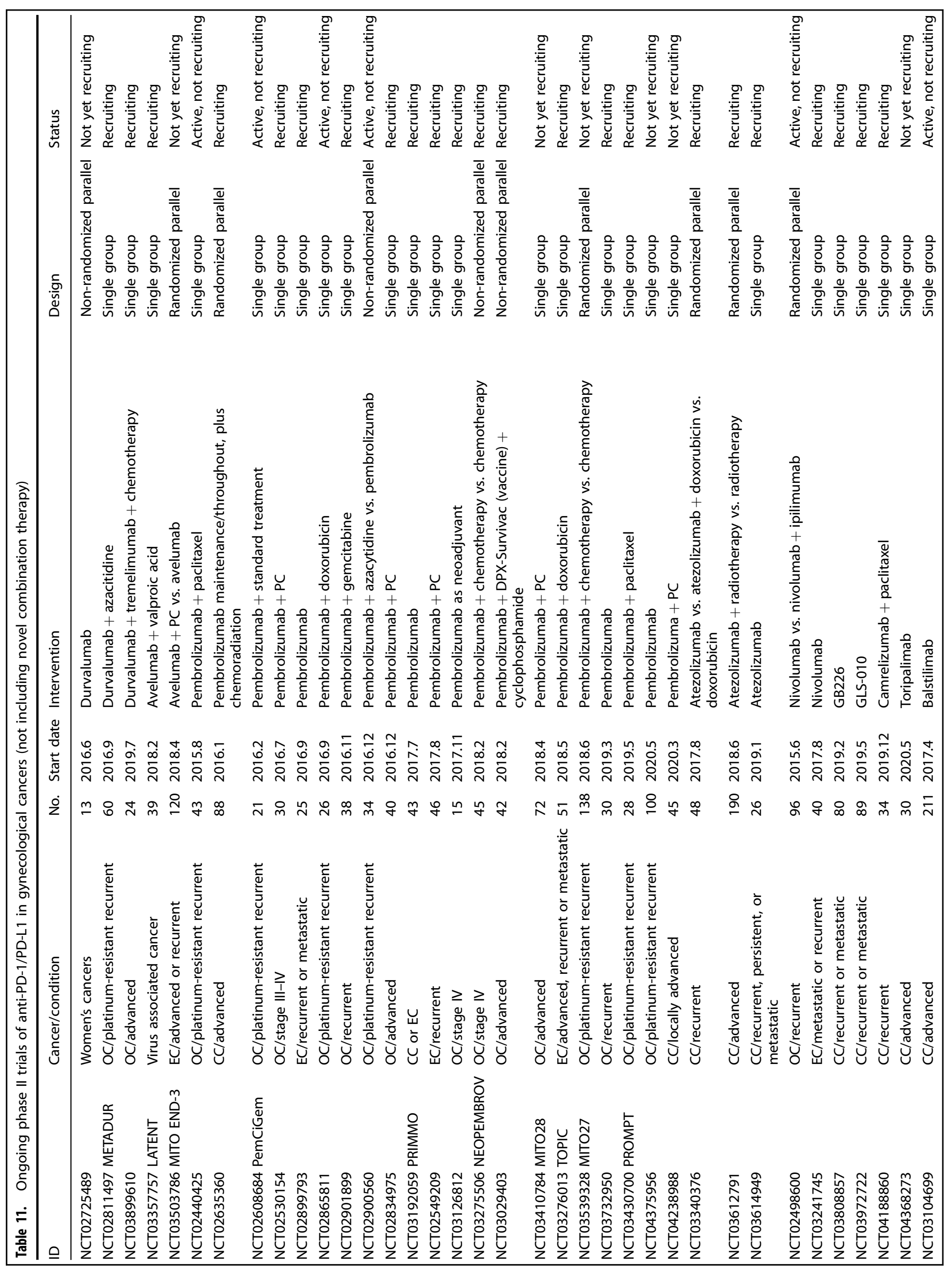


Table 12. Ongoing phase III trials of anti-PD-1/PD-L1 in gynecological cancers (not including novel combination therapy)

\begin{tabular}{|c|c|c|c|c|c|}
\hline ID & Cancer/condition & No. & Start date & Intervention & Status \\
\hline NCT03038100 IMagyn050 & OC/stage III-IV & 1300 & 2017.3 & $\begin{array}{l}\text { Atezolizumab vs. placebo, plus PC }+ \\
\text { bevacizumab }\end{array}$ & $\begin{array}{l}\text { Active, not } \\
\text { recruiting }\end{array}$ \\
\hline NCT03556839 & $\mathrm{CC} /$ stage IVb & 404 & 2018.9 & $\begin{array}{l}\text { Atezolizumab vs. placebo, plus PC }+ \\
\text { bevacizumab }\end{array}$ & Recruiting \\
\hline NCT03603184 AtTEnd & EC/advanced & 550 & 2018.10 & Atezolizumab vs. placebo, plus PC & Recruiting \\
\hline NCT03635567 KEYNOTE-826 & $\mathrm{CC} /$ persistent, recurrent, or metastatic & 600 & 2018.10 & $\begin{array}{l}\text { Pembrolizumab vs. placebo, plus PC }+ \\
\text { bevacizumab }\end{array}$ & Recruiting \\
\hline NCT03914612 & $\mathrm{EC} /$ advanced or recurrent & 810 & 2019.7 & Pembrolizumab vs. placebo, plus PC & Recruiting \\
\hline NCT03981796 RUBY & $\mathrm{EC} /$ recurrent or stage III-IV & 470 & 2019.7 & Dostarlimab vs. placebo, plus PC & Recruiting \\
\hline NCT03912415 FERMATA & CC/advanced & 316 & 2019.9 & $\begin{array}{l}\text { Prolgolimab vs. placebo, plus PC }+ \\
\text { bevacizumab }\end{array}$ & $\begin{array}{l}\text { Not yet } \\
\text { recruiting }\end{array}$ \\
\hline
\end{tabular}

Table 13. Ongoing phase III trials of novel combination targeted therapy in gynecological cancers

\begin{tabular}{|c|c|c|c|c|c|c|}
\hline ID & Cancer/condition & No. & Start date & Target & Intervention & Status \\
\hline NCT03522246 ATHENA & OC/stage III-IV & 1012 & 2018.5 & PARP, PD-1 & $\begin{array}{l}\text { Rucaparib + nivolumab vs. rucaparib + placebo vs. } \\
\text { nivolumab + placebo vs. placebo }\end{array}$ & Recruiting \\
\hline $\begin{array}{l}\text { NCT03884101 ENGOT- } \\
\text { en9 }\end{array}$ & $\mathrm{EC} /$ recurrent or stage III-IV & 720 & 2019.4 & VEGF, PD-1 & Lenvatinib + pembrolizumab vs. chemotherapy & Recruiting \\
\hline $\begin{array}{l}\text { NCT03740165 } \\
\text { KEYLYNK-001/ENGOT- } \\
\text { ov43 }\end{array}$ & OC/fist-line treatment & 1086 & 2018.12 & $\begin{array}{l}\text { VEGF, PARP, } \\
\text { PD-1 }\end{array}$ & $\begin{array}{l}\text { Pembrolizumab }+ \text { olaparib vs. pembrolizumab }+ \\
\text { placebo vs. placebo, plus PC }+ \text { bevacizumab }\end{array}$ & Recruiting \\
\hline NCT03737643 DUO-O & OC/stage III-IV & 1056 & 2019.1 & $\begin{array}{l}\text { VEGF, PARP, } \\
\text { PD-1 }\end{array}$ & $\begin{array}{l}\text { Durvalumab }+ \text { olaparib vs. durvalumab }+ \text { placebo } \\
\text { vs. placebo, plus } P C+\text { bevacizumab }\end{array}$ & Recruiting \\
\hline
\end{tabular}

fulvestrant in advanced, recurrent, or persistent EC. ${ }^{394}$ No patient demonstrated a complete or partial response in the 22 ERnegative patients, with a stable disease rate of $18 \%$ as the best response. The median PFS and OS were 2 and 3 months, respectively. In the 31 ER-positive patients, the ORR and stable disease rate were $16 \%$ and $29 \%$, with a median PFS of 10 months and a median OS of 26 months, respectively. As for OC, fulvestrant was associated with a low ORR of $8 \%$ and a stable disease rate of $35 \%$ in ER-positive, multiply recurrent OC. ${ }^{395}$ The effect of antiestrogenic agents in advanced or recurrent EC needs further investigations. Furthermore, combining hormonal therapy with targeted therapies is a novel strategy in treating certain gynecological cancers, which is being assessed in several ongoing clinical trials (e.g., NCT03643510, NCT03294694, NCT02730923, NCT02476955, and NCT02188550).

\section{CONCLUSION}

From the large amount of clinical trials on targeted agents and molecular drugs, we can see the great enthusiasm in targeted therapies. Consequently, it has led to significant breakthrough in personalized medicine of antitumor treatment strategy, including gynecological cancers. According to current clinical evidence, PARP inhibitors have made a remarkable progress in treatment of OC depending on the identification of disease with HRD (e.g., BRCAm). As for EC, given the identification of hormone-dependent histological type and POLE/MSI molecular subtypes, the activity of $\mathrm{PI} 3 \mathrm{~K} / \mathrm{AKT} / \mathrm{mTOR}, \mathrm{PD}-1$, and hormone receptor-targeted therapies might be promising in treatment of patients with EC. Since CC is mostly associated with persistent infection of virus, immunetargeted therapies (e.g., anti-PD-1/PD-L1 agents) are expected to be prospective treatment strategy. For the future research, as we 


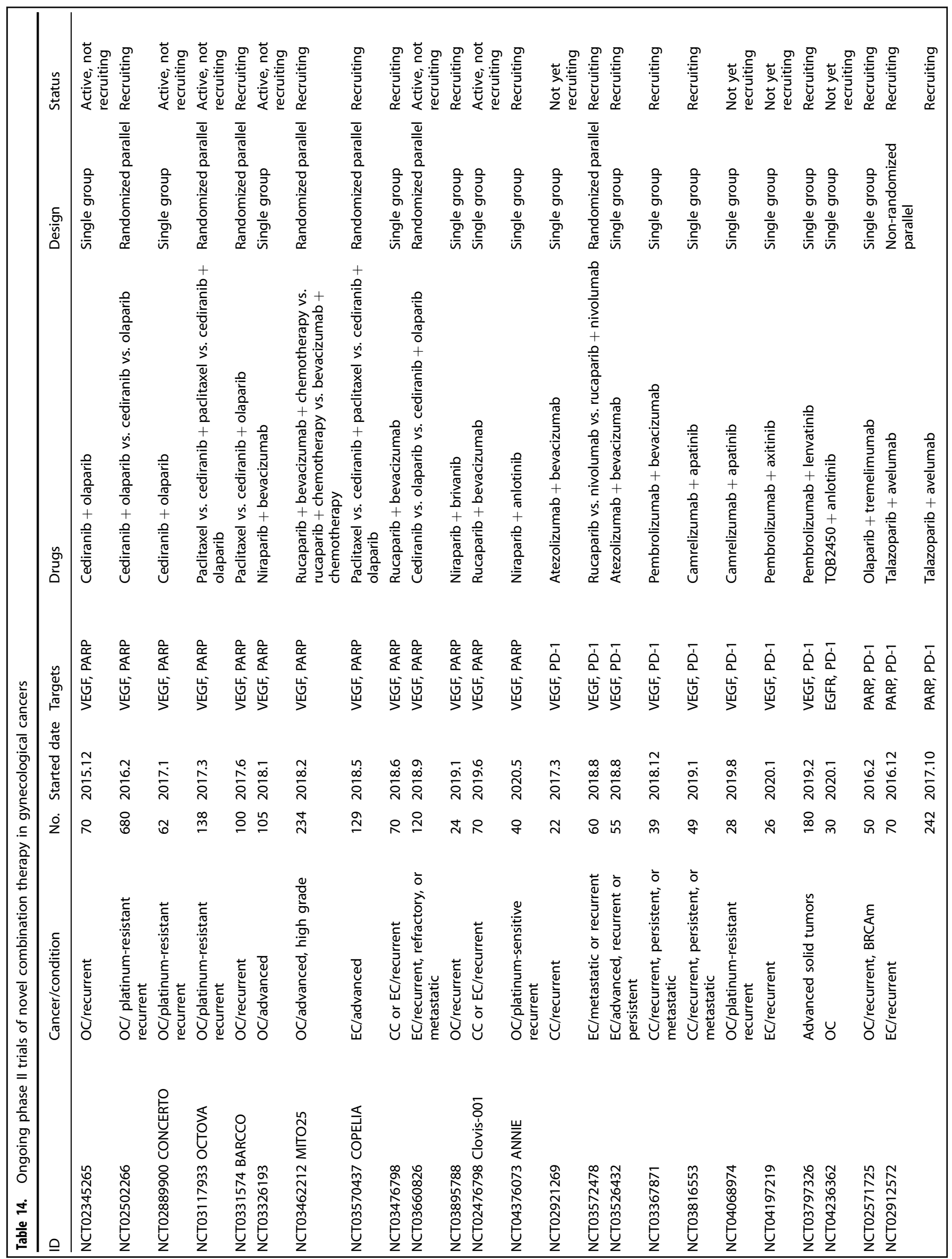




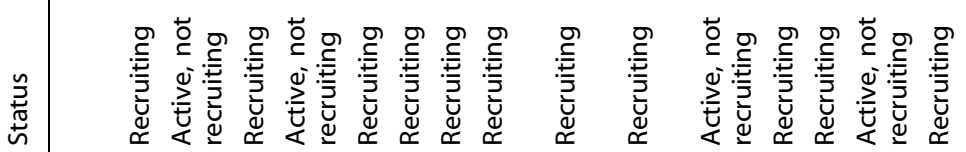

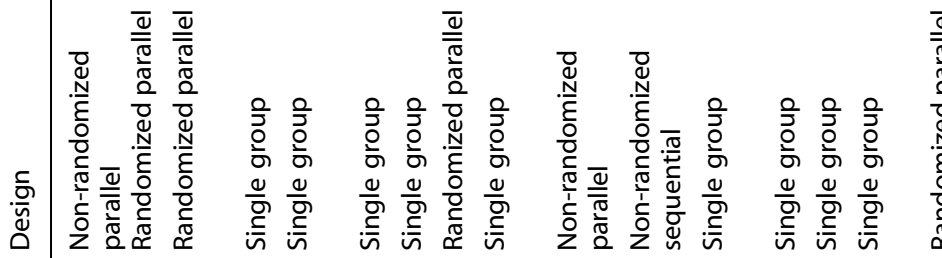

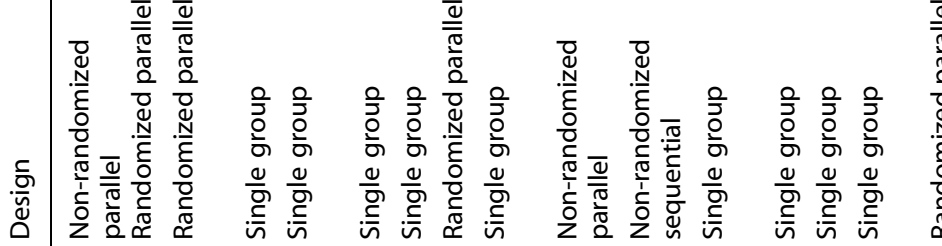

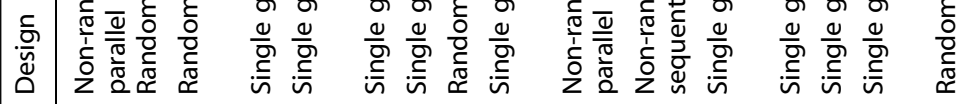

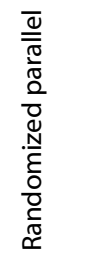

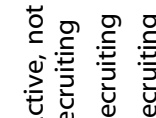

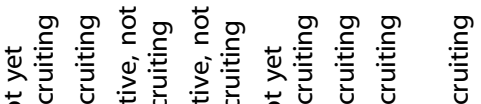

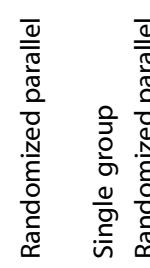

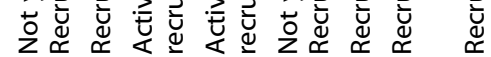

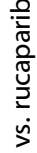

芩

常

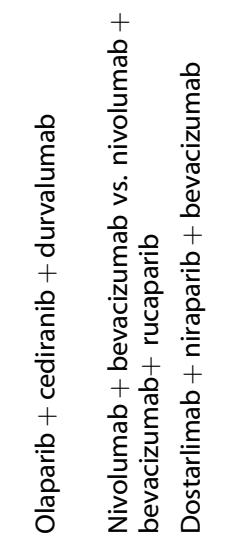

$\dot{s}$

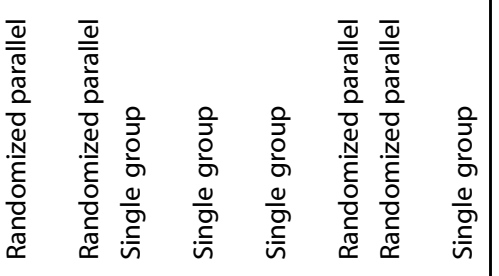

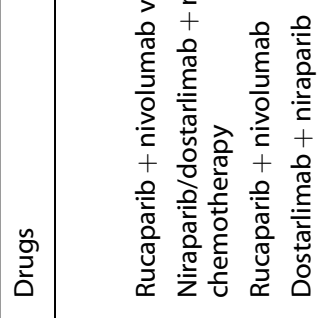

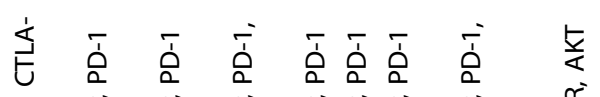

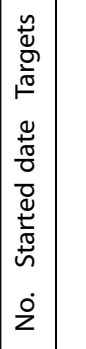

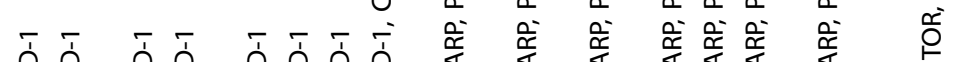

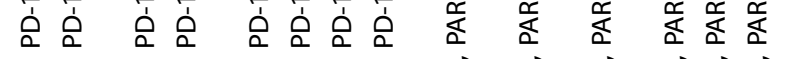

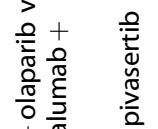

: $\frac{\pi}{\mathrm{O}}$

$\dot{s} \cdot \frac{1}{\bar{c}}$

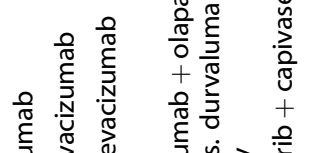

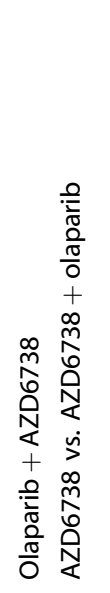

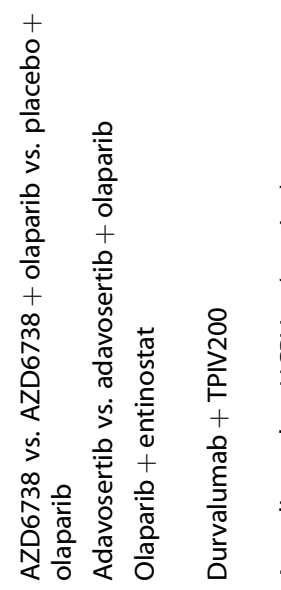

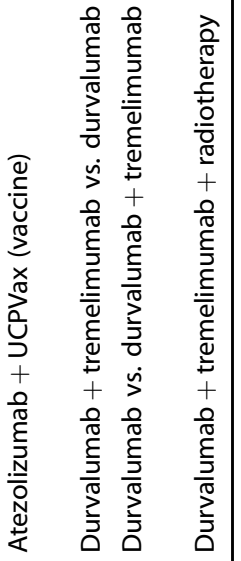

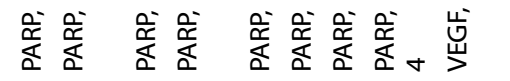

崖

舟

요

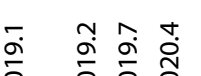

$\frac{\bar{\infty}}{\infty}$

$\sqrt{2}$

$\circ \stackrel{\circ}{\circ} \stackrel{ }{ }$

นn $\infty \stackrel{\circ}{\circ}$ m

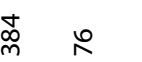

F

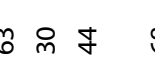

爱变

安

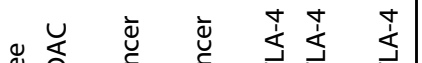

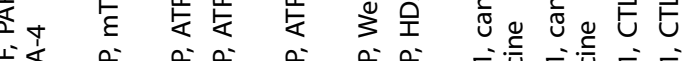

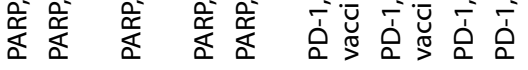

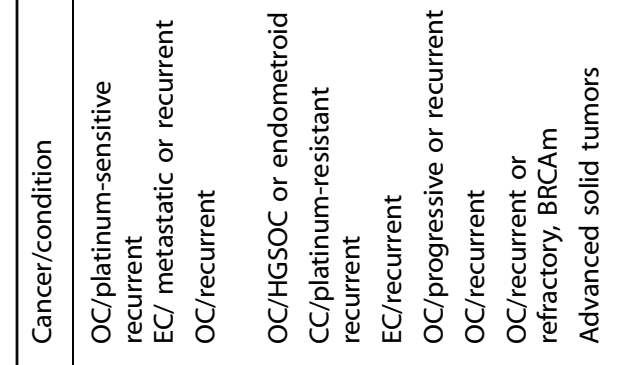

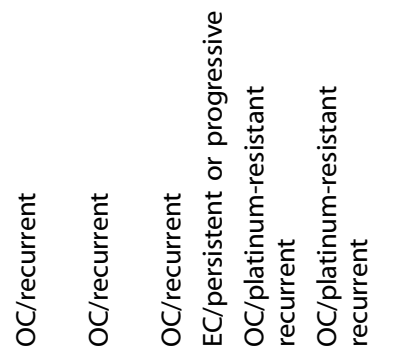

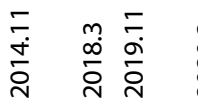

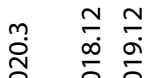

กุ

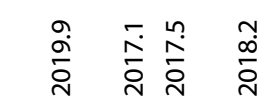

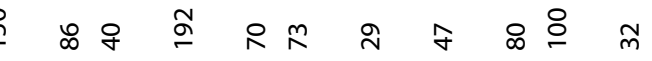

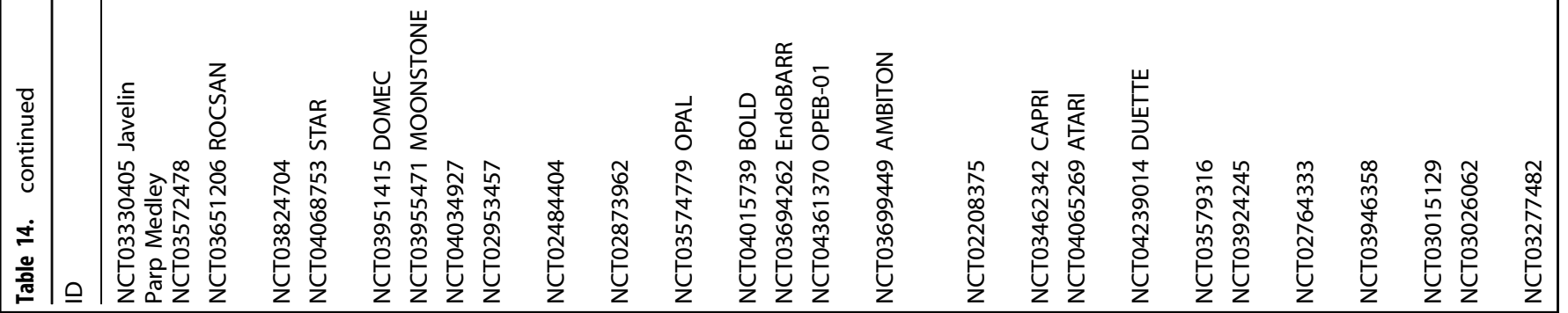


discussed previously, specific biomarkers are the keys to the tumor response of targeted drugs. Moreover, novel combination therapies, coinhibition of different targets, are worth conducting to overcome the drug resistance in cancer cells. A number of phase II/III clinical trials of novel combination strategies have been in progress (Tables 13 and 14).

\section{ACKNOWLEDGEMENTS}

This study was supported by National Major Scientific and Technological Project for "Significant New Drugs Development" in China (2018ZX09733001).

\section{AUTHOR CONTRIBUTIONS}

X.Z. substantially contributed to the conception and design of the work. Q.W. and H.L. P. did the literature research and data retrieval. Q.W. drafted the article and revised it. X.R.Q. and M.W. reviewed the draft. All authors approved the submitted version.

\section{ADDITIONAL INFORMATION}

Competing interests: The authors declare no competing interests.

\section{REFERENCES}

1. Siegel, R., Miller, K. \& Jemal, A. Cancer statistics, 2019. CA: Cancer J. Clin. 69, 7-34 (2019).

2. Matulonis, U. A. et al. Ovarian cancer. Nat. Rev. Dis. Prim. 2, 16061 (2016).

3. Jelovac, D. \& Armstrong, D. K. Recent progress in the diagnosis and treatment of ovarian cancer. CA: Cancer J. Clin. 61, 183-203 (2011).

4. Bowtell, D. D. et al. Rethinking ovarian cancer II: reducing mortality from highgrade serous ovarian cancer. Nat. Rev. Cancer 15, 668 (2015).

5. Bristow, R. E. et al. Survival impact of surgical cytoreduction in stage IV epithelial ovarian cancer. Gynecol. Oncol. 72, 278-287 (1999).

6. Reid, B. M., Permuth, J. B. \& Sellers, T. A. Epidemiology of ovarian cancer: a review. Cancer Biol. Med. 14, 9-32 (2017).

7. Colombo, N. et al. Newly diagnosed and relapsed epithelial ovarian carcinoma: ESMO Clinical Practice Guidelines for diagnosis, treatment and follow-up. Ann. Oncol. Suppl 5, v23-v30 (2010).

8. Christie, E. L. \& Bowtell, D. D. L. Acquired chemotherapy resistance in ovarian cancer. Ann. Oncol. 28, viii13 (2017).

9. Pignata, S. et al. Treatment of recurrent epithelial ovarian cancer. Cancer 125 (Suppl 24), 4609-4615 (2019).

10. Bray, F. et al. Global cancer statistics 2018: GLOBOCAN estimates of incidence and mortality worldwide for 36 cancers in 185 countries. CA: a cancer J. clinicians. 68, 394-424 (2018)

11. Munoz, N. et al. Epidemiologic classification of human papillomavirus types associated with cervical cancer. N. Engl. J. Med. 348, 518-527 (2003).

12. Vande Pol, S. B. \& Klingelhutz, A. J. Papillomavirus E6 oncoproteins. Virology 445 115-137 (2013).

13. Roman, A. \& Munger, K. The papillomavirus E7 proteins. Virology 445, 138-168 (2013).

14. Future II Study Group. Quadrivalent vaccine against human papillomavirus to prevent high-grade cervical lesions. N. England J. Med. 356, 1915-1927 (2007)

15. Wentzensen, N., Schiffman, M., Palmer, T. \& Arbyn, M. Triage of HPV positive women in cervical cancer screening. J. Clin. Virol. 76, S49-S55 (2016).

16. Morice, P. et al. Endometrial cancer. Lancet 387, 1094-1108 (2016).

17. Renehan, A. G. et al. Body-mass index and incidence of cancer: a systematic review and meta-analysis of prospective observational studies. Lancet $\mathbf{3 7 1}$ 569-578 (2008)

18. Colombo, N. et al. ESMO-ESGO-ESTRO consensus conference on endometrial cancer: diagnosis, treatment and follow-up. Int. J. Gynecol. Cancer 26, 2-30 (2016).

19. Stelloo, E. et al. Refining prognosis and identifying targetable pathways for high-risk endometrial cancer; a TransPORTEC initiative. Mod. Pathol. 28, 836-844 (2015).

20. Kandoth, C. et al. Integrated genomic characterization of endometrial carcinoma. Nature 497, 67-73 (2013)

21. Lee, Y. C., Lheureux, S. \& Oza, A. M. Treatment strategies for endometrial cancer: current practice and perspective. Curr. Opin. Obstet. Gynecol. 29, 47-58 (2017).

22. Butler, J. M., Kobayashi, H. \& Rafii, S. Instructive role of the vascular niche in promoting tumour growth and tissue repair by angiocrine factors. Nat. Rev. Cancer 10, 138-146 (2010).

23. Siemann, D. W. et al. Differentiation and definition of vascular-targeted therapies. Clin. Cancer Res. 11, 416-420 (2005). 
24. Ferrara, N. \& Kerbel, R. S. Angiogenesis as a therapeutic target. Nature 438, 967-974 (2005).

25. Viallard, C. \& Larrivee, B. Tumor angiogenesis and vascular normalization: alternative therapeutic targets. Angiogenesis 20, 409-426 (2017).

26. Bridges, E. M. \& Harris, A. L. The angiogenic process as a therapeutic target in cancer. Biochem. Pharmacol. 81, 1183-1191 (2011).

27. Miyasaka, A. et al. PI3K/mTOR pathway inhibition overcomes radioresistance via suppression of the HIF1-a/VEGF pathway in endometrial cancer. Gynecol. Oncol. 138, 174-180 (2015)

28. Graybill, W., Sood, A. K., Monk, B. J. \& Coleman, R. L. State of the science: emerging therapeutic strategies for targeting angiogenesis in ovarian cancer. Gynecologic Oncol. 138, 223-226 (2015).

29. Jayson, G. C., Kerbel, R., Ellis, L. M. \& Harris, A. L. Antiangiogenic therapy in oncology: current status and future directions. Lancet 388, 518-529 (2016).

30. Kerbel, R. S. Tumor. Angiogenesis 358, 2039-2049 (2008).

31. Camerin, G. R. et al. VEGF gene polymorphisms and outcome of epithelial ovarian cancer patients. Future Oncol. 13, 409-414 (2017).

32. Vergote, l. et al. A phase $1 \mathrm{~b}$ study of trebananib in combination with pegylated liposomal doxorubicin or topotecan in women with recurrent platinum-resistant or partially platinum-sensitive ovarian cancer. Gynecol. Oncol. 135, 25-33 (2014).

33. Haqshenas, G. \& Doerig, C. Targeting of host cell receptor tyrosine kinases by intracellular pathogens. Sci. Signal. 12, eaau9894 (2019).

34. Burger, R. A. et al. Incorporation of bevacizumab in the primary treatment of ovarian cancer. N. Engl. J. Med. 365, 2473-2483 (2011).

35. Tewari, K. S. et al. Final overall survival of a randomized trial of bevacizumab for primary treatment of ovarian. Cancer 37, 2317-2328 (2019).

36. Perren, T. J. et al. A phase 3 trial of bevacizumab in ovarian cancer. N. Engl. J. Med. 365, 2484-2496 (2011).

37. Oza, A. M. et al. Standard chemotherapy with or without bevacizumab for women with newly diagnosed ovarian cancer (ICON7): overall survival results of a phase 3 randomised trial. Lancet Oncol. 16, 928-936 (2015).

38. Friedlander, M. et al. Health-related quality of life and patient-centred outcomes with olaparib maintenance after chemotherapy in patients with platinum-sensitive, relapsed ovarian cancer and a BRCA1/2 mutation (SOLO2/ENGOT Ov-21): a placebo-controlled, phase 3 randomised trial. Lancet Oncol. 19, 1126-1134 (2018).

39. Aghajanian, C. et al. OCEANS: a randomized, double-blind, placebo-controlled phase III trial of chemotherapy with or without bevacizumab in patients with platinum-sensitive recurrent epithelial ovarian, primary peritoneal, or fallopian tube cancer. J. Clin. Oncol. 30, 2039-2045 (2012).

40. Aghajanian, C. et al. Final overall survival and safety analysis of OCEANS, a phase 3 trial of chemotherapy with or without bevacizumab in patients with platinumsensitive recurrent ovarian cancer. Gynecol. Oncol. 139, 10-16 (2015).

41. Coleman, R. L. et al. Bevacizumab and paclitaxel-carboplatin chemotherapy and secondary cytoreduction in recurrent, platinum-sensitive ovarian cancer (NRG Oncology/Gynecologic Oncology Group study GOG-0213): a multicentre, openlabel, randomised, phase 3 trial. Lancet Oncol. 18, 779-791 (2017).

42. Bamias, A. et al. Bevacizumab with or after chemotherapy for platinum-resistant recurrent ovarian cancer: exploratory analyses of the AURELIA trial. Ann. Oncol. 28, 1842-1848 (2017).

43. Pujade-Lauraine, E. et al. Bevacizumab combined with chemotherapy for platinum-resistant recurrent ovarian cancer: the AURELIA open-label randomized phase III trial. J. Clin. Oncol. 32, 1302-1308 (2014).

44. Gore, M. et al. An international, phase III randomized trial in patients with mucinous epithelial ovarian cancer (mEOC/GOG 0241) with long-term follow-up: and experience of conducting a clinical trial in a rare gynecological tumor. Gynecol. Oncol. 153, 541-548 (2019).

45. Monk, B. J. et al. Phase II trial of bevacizumab in the treatment of persistent or recurrent squamous cell carcinoma of the cervix: a gynecologic oncology group study. J. Clin. Oncol. 27, 1069-1074 (2009).

46. Zighelboim, I. et al. Multicenter phase II trial of topotecan, cisplatin and bevacizumab for recurrent or persistent cervical cancer. Gynecol. Oncol. 130, 64-68 (2013).

47. Suzuki, K. et al. Phase II trial of paclitaxel, carboplatin, and bevacizumab for advanced or recurrent cervical cancer. Gynecol. Oncol. 154, 554-557 (2019).

48. Tewari, K. S. et al. Bevacizumab for advanced cervical cancer: final overall survival and adverse event analysis of a randomised, controlled, open-label, phase 3 trial (Gynecologic Oncology Group 240). Lancet 390, 1654-1663 (2017).

49. Schefter, T. E. et al. A phase II study of bevacizumab in combination with definitive radiotherapy and cisplatin chemotherapy in untreated patients with locally advanced cervical carcinoma: preliminary results of RTOG 0417. Int. J. Radiat. Oncol. Biol. Phys. 83, 1179-1184 (2012)

50. Ishikawa, M. et al. A randomized phase II/III trial of conventional paclitaxel and carboplatin with or without bevacizumab vs dose-dense paclitaxel and carboplatin with or without bevacizumab, in stage IVB, recurrent or persistent cervical carcinoma: Japan Clinical Oncology Group Study (JCOG1311). Jpn J. Clin. Oncol. 48, 1096-1100 (2018).

51. Aghajanian, C. et al. Phase II trial of bevacizumab in recurrent or persistent endometrial cancer: a Gynecologic Oncology Group Study. J. Clin. Oncol. 29, 2259-2265 (2011).

52. Simpkins, F. et al. A phase II trial of paclitaxel, carboplatin, and bevacizumab in advanced and recurrent endometrial carcinoma (EMCA). Gynecol. Oncol. 136, 240-245 (2015).

53. Viswanathan, A. N. et al. NRG Oncology/RTOG 0921: a phase 2 study of postoperative intensity-modulated radiotherapy with concurrent cisplatin and bevacizumab followed by carboplatin and paclitaxel for patients with endometrial cancer. Cancer 121, 2156-2163 (2015).

54. Lorusso, D. et al. Carboplatin-paclitaxel compared to carboplatin-paclitaxelbevacizumab in advanced or recurrent endometrial cancer: MITO END-2-a randomized phase II trial. Gynecol. Oncol. 155, 406-412 (2019).

55. Oza, A. M. et al. Efficacy and safety of bevacizumab-containing therapy in newly diagnosed ovarian cancer: ROSiA single-arm phase 3B study. Int. J. Gynecol. Cancer 27, 50-58 (2017).

56. Verheijen, R. B. et al. Clinical pharmacokinetics and pharmacodynamics of pazopanib: towards optimized dosing. Clin. Pharmacokinet. 56, 987-997 (2017).

57. Kerklaan, B. M. et al. Phase I and pharmacological study of pazopanib in combination with oral topotecan in patients with advanced solid tumours. Br. J. Cancer 113, 706-715 (2015).

58. Hainsworth, J. D., Firdaus, I. D., Earwood, C. B. \& Chua, C. C. Pazopanib and liposomal doxorubicin in the treatment of patients with relapsed/refractory epithelial ovarian cancer: a phase lb study of the Sarah Cannon Research Institute. Cancer Investig. 33, 47-52 (2015).

59. Friedlander, M. et al. A Phase II, open-label study evaluating pazopanib in patients with recurrent ovarian cancer. Gynecol. Oncol. 119, 32-37 (2010).

60. Pignata, S. et al. Pazopanib plus weekly paclitaxel versus weekly paclitaxel alone for platinum-resistant or platinum-refractory advanced ovarian cancer (MITO 11): a randomised, open-label, phase 2 trial. Lancet Oncol. 16, 561-568 (2015).

61. Dinkic, C. et al. Pazopanib (GW786034) and cyclophosphamide in patients with platinum-resistant, recurrent, pre-treated ovarian cancer-results of the PACOVAR-trial. Gynecol. Oncol. 146, 279-284 (2017).

62. du Bois, A. et al. Incorporation of pazopanib in maintenance therapy of ovarian cancer. J. Clin. Oncol. 32, 3374-3382 (2014).

63. Vergote, I. et al. Overall survival results of AGO-OVAR16: a phase 3 study of maintenance pazopanib versus placebo in women who have not progressed after first-line chemotherapy for advanced ovarian cancer. Gynecol. Oncol. 155, 186-191 (2019).

64. Morgan, R. D. et al. Pazopanib and fosbretabulin in recurrent ovarian cancer (PAZOFOS): a multi-centre, phase $1 \mathrm{~b}$ and open-label, randomised phase 2 trial. Gynecol. Oncol. 156, 545-551 (2020).

65. Campos, S. M. et al. A phase II evaluation of pazopanib in the treatment of recurrent or persistent carcinosarcoma of the uterus: a gynecologic oncology group study. Gynecol. Oncol. 133, 537-541 (2014).

66. Ledermann, J. A. et al. Randomized phase II placebo-controlled trial of maintenance therapy using the oral triple angiokinase inhibitor BIBF 1120 after chemotherapy for relapsed ovarian cancer. J. Clin. Oncol. 29, 3798-3804 (2011).

67. du Bois, A. et al. Standard first-line chemotherapy with or without nintedanib for advanced ovarian cancer (AGO-OVAR 12): a randomised, double-blind, placebocontrolled phase 3 trial. Lancet Oncol. 17, 78-89 (2016).

68. Secord, A. A. et al. Phase II trial of nintedanib in patients with bevacizumabresistant recurrent epithelial ovarian, tubal, and peritoneal cancer. Gynecol. Oncol. 153, 555-561 (2019).

69. Dizon, D. S. et al. A phase II evaluation of nintedanib (BIBF-1120) in the treatment of recurrent or persistent endometrial cancer: an NRG Oncology/Gynecologic Oncology Group Study. Gynecol. Oncol. 135, 441-445 (2014).

70. Tang, W., McCormick, A., Li, J. \& Masson, E. Clinical pharmacokinetics and pharmacodynamics of cediranib. Clin. Pharmacokinet. 56, 689-702 (2017).

71. Kaplan, A. R. et al. Cediranib suppresses homology-directed DNA repair through down-regulation of BRCA1/2 and RAD51. Sci. Transl. Med. 11, eaav4508 (2019).

72. Hirte, $\mathrm{H}$. et al. A phase 2 study of cediranib in recurrent or persistent ovarian, peritoneal or fallopian tube cancer: a trial of the Princess Margaret, Chicago and California Phase II Consortia. Gynecol. Oncol. 138, 55-61 (2015).

73. Ledermann, J. A. et al. Cediranib in patients with relapsed platinum-sensitive ovarian cancer (ICON6): a randomised, double-blind, placebo-controlled phase 3 trial. Lancet 387, 1066-1074 (2016).

74. Bender, D. et al. A phase II evaluation of cediranib in the treatment of recurrent or persistent endometrial cancer: an NRG Oncology/Gynecologic Oncology Group study. Gynecol. Oncol. 138, 507-512 (2015).

75. Gerald, D., Chintharlapalli, S., Augustin, H. G. \& Benjamin, L. E. Angiopoietin-2: an attractive target for improved antiangiogenic tumor therapy. Cancer Res. 73, 1649-1657 (2013). 
Targeted therapies in gynecological cancers: a comprehensive review of... Wang et al.

76. Karlan, B. Y. et al. Randomized, double-blind, placebo-controlled phase II study of AMG 386 combined with weekly paclitaxel in patients with recurrent ovarian cancer. Lancet Oncol. 30, 362-371 (2012).

77. Coxon, A. et al. Context-dependent role of angiopoietin-1 inhibition in the suppression of angiogenesis and tumor growth: implications for AMG 386, an angiopoietin-1/2-neutralizing peptibody. Mol. Cancer Ther. 9, 2641-2651 (2010).

78. Monk, B. J. et al. Anti-angiopoietin therapy with trebananib for recurrent ovarian cancer (TRINOVA-1): a randomised, multicentre, double-blind, placebocontrolled phase 3 trial. Lancet Oncol. 15, 799-808 (2014).

79. Stark, D. P. et al. Quality of life with cediranib in relapsed ovarian cancer: The ICON6 phase 3 randomized clinical trial. Cancer 123, 2752-2761 (2017).

80. Vergote, I. et al. Trebananib or placebo plus carboplatin and paclitaxel as firstline treatment for advanced ovarian cancer (TRINOVA-3/ENGOT-ov2/GOG-3001): a randomised, double-blind, phase 3 trial. Lancet Oncol. 20, 862-876 (2019).

81. Marth, C. et al. ENGOT-ov-6/TRINOVA-2: Randomised, double-blind, phase 3 study of pegylated liposomal doxorubicin plus trebananib or placebo in women with recurrent partially platinum-sensitive or resistant ovarian cancer. Eur. J. Cancer 70, 111-121 (2017)

82. Moore, K. N. et al. A phase II trial of trebananib (AMG 386; IND\#111071), a selective angiopoietin $1 / 2$ neutralizing peptibody, in patients with persistent/ recurrent carcinoma of the endometrium: an NRG/Gynecologic Oncology Group trial. Gynecol. Oncol. 138, 513-518 (2015).

83. Zhao, D., Hou, H. \& Zhang, X. Progress in the treatment of solid tumors with apatinib: a systematic review. OncoTargets Ther. 11, 4137-4147 (2018).

84. Jin, M. et al. Successful maintenance therapy with apatinib inplatinum-resistant advanced ovarian cancer and literature review. Cancer Biol. Ther. 19, 1088-1092 (2018).

85. Li, J. et al. Randomized, double-blind, placebo-controlled phase III trial of apatinib in patients with chemotherapy-refractory advanced or metastatic adenocarcinoma of the stomach or gastroesophageal junction. J. Clin. Oncol. 34, 1448-1454 (2016).

86. Miao, M. et al. A phase II study of apatinib in patients with recurrent epithelial ovarian cancer. Gynecol. Oncol. 148, 286-290 (2018).

87. Lan, C. Y. et al. Apatinib combined with oral etoposide in patients with platinum-resistant or platinum-refractory ovarian cancer (AEROC): a phase 2, single-arm, prospective study. Lancet Oncol. 19, 1239-1246 (2018).

88. Campos, S. M. et al. A phase II trial of Sunitinib malate in recurrent and refractory ovarian, fallopian tube and peritoneal carcinoma. Gynecol. Oncol. 128, 215-220 (2013).

89. Baumann, K. H. et al. A phase II trial (AGO 2.11) in platinum-resistant ovarian cancer: a randomized multicenter trial with sunitinib (SU11248) to evaluate dosage, schedule, tolerability, toxicity and effectiveness of a multitargeted receptor tyrosine kinase inhibitor monotherapy. Ann. Oncol. 23, 2265-2271 (2012).

90. Biagi, J. J. et al. A phase II study of sunitinib in patients with recurrent epithelial ovarian and primary peritoneal carcinoma: an NCIC Clinical Trials Group Study. Ann. Oncol. 22, 335-340 (2011).

91. Chan, J. K. et al. A phase II evaluation of sunitinib in the treatment of persistent or recurrent clear cell ovarian carcinoma: an NRG Oncology/Gynecologic Oncology Group Study (GOG-254). Gynecol. Oncol. 150, 247-252 (2018).

92. Castonguay, V. et al. A phase II trial of sunitinib in women with metastatic or recurrent endometrial carcinoma: a study of the Princess Margaret, Chicago and California Consortia. Gynecol. Oncol. 134, 274-280 (2014).

93. Mackay, H. J. et al. A phase II study of sunitinib in patients with locally advanced or metastatic cervical carcinoma: NCIC CTG Trial IND.184. Gynecol. Oncol. 116 163-167 (2010).

94. Chan, J. K. et al. A phase II evaluation of brivanib in the treatment of persistent or recurrent carcinoma of the cervix: an NRG Oncology/Gynecologic Oncology Group study. Gynecol. Oncol. 146, 554-559 (2017).

95. Powell, M. A. et al. A phase II trial of brivanib in recurrent or persistent endometrial cancer: an NRG Oncology/Gynecologic Oncology Group Study. Gynecol. Oncol. 135, 38-43 (2014).

96. Curtin, N. J. \& Szabo, C. Therapeutic applications of PARP inhibitors: anticancer therapy and beyond. Mol. Asp. Med. 34, 1217-1256 (2013).

97. Michèle, R. et al. PARP inhibition: PARP1 beyond. Nat. Rev. Cancer 10, 293-301 (2010).

98. Helleday, T., Lo, J., van Gent, D. C. \& Engelward, B. P. DNA double-strand break repair: from mechanistic understanding to cancer treatment. DNA Repair $\mathbf{6}$, 923-935 (2007)

99. Scott, C. L., Swisher, E. M. \& Kaufmann, S. H. Poly (ADP-ribose) polymerase inhibitors: recent advances and future development. J. Clin. Oncol. 33, 1397-1406 (2015).

100. Takata, M. et al. Homologous recombination and non-homologous end-joining pathways of DNA double-strand break repair have overlapping roles in the maintenance of chromosomal integrity in vertebrate cells. $E M B O$ J. 17, 5497-5508 (1998).

101. Gudmundsdottir, K. \& Ashworth, A. The roles of BRCA1 and BRCA2 and associated proteins in the maintenance of genomic stability. Oncogene $\mathbf{2 5}$ 5864-5874 (2006).

102. Drew, Y. The development of PARP inhibitors in ovarian cancer: from bench to bedside. Br. J. Cancer 113, S3-S9 (2015).

103. Bellio, C. et al. PARP inhibition induces enrichment of DNA repair-proficient CD133 and CD117 positive ovarian cancer. Stem Cells 17, 431-445 (2019).

104. Kathryn, A. et al. BRCA mutation frequency and patterns of treatment response in BRCA mutation-positive women with ovarian cancer: a report from the Australian Ovarian Cancer Study Group. J. Clin. Oncol. 30, 2654-2663 (2012).

105. Network Atlas, T.C.G. Integrated Genomic analyses of ovarian carcinoma. Nature 474, 292-292 (2011).

106. Cancer Genome Atlas Research Network. integrated genomic analyses of ovarian carcinoma. Nature 474, 609-615 (2011).

107. Konstantinopoulos, P. A., Ceccaldi, R., Shapiro, G. I. \& D'Andrea, A. D. Homologous recombination deficiency: exploiting the fundamental vulnerability of ovarian cancer. Cancer Discov. 5, 1137-1154 (2015).

108. Ang, Y. L. \& Tan, D. S. Development of PARP inhibitors in gynecological malignancies. Curr. Prob. Cancer 41, 273-286 (2017).

109. Donawho, C. K. et al. ABT-888, an orally active poly(ADP-ribose) polymerase inhibitor that potentiates DNA-damaging agents in preclinical tumor models. Clin. Cancer Res. 13, 2728-2737 (2007).

110. Mirza, M. R., Pignata, S. \& Ledermann, J. A. Latest clinical evidence and further development of PARP inhibitors in ovarian cancer. Ann. Oncol. 29, 1366-1376 (2018).

111. Gelmon, K. A. et al. Olaparib in patients with recurrent high-grade serous or poorly differentiated ovarian carcinoma or triple-negative breast cancer: a phase 2, multicentre, open-label, non-randomised study. Lancet Oncol. 12, 852-861 (2011).

112. Fong, P. C. et al. Inhibition of poly(ADP-ribose) polymerase in tumors from BRCA mutation carriers. N. Engl. J. Med. 361, 123-134 (2009).

113. Lee, C. K. et al. Phase 1 trial of olaparib and oral cyclophosphamide in BRCA breast cancer, recurrent BRCA ovarian cancer, non-BRCA triple-negative breast cancer, and non-BRCA ovarian cancer. Br. J. Cancer 120, 279-285 (2019).

114. Ang, J. E. et al. Efficacy of chemotherapy in BRCA1/2 mutation carrier ovarian cancer in the setting of PARP inhibitor resistance: a multi-institutional study. Clin. Cancer Res. 19, 5485-5493 (2013).

115. Jonathan, L. et al. Olaparib maintenance therapy in platinum-sensitive relapsed ovarian cancer. N. Engl. J. Med. 366, 1382-1392 (2012).

116. Matulonis, U. A. et al. Olaparib maintenance therapy in patients with platinumsensitive, relapsed serous ovarian cancer and a BRCA mutation: overall survival adjusted for postprogression poly(adenosine diphosphate ribose) polymerase inhibitor therapy. Cancer 122, 1844-1852 (2016).

117. Ledermann, J. et al. Olaparib maintenance therapy in patients with platinumsensitive relapsed serous ovarian cancer: a preplanned retrospective analysis of outcomes by BRCA status in a randomised phase 2 trial. Lancet Oncol. 15, 852-861 (2014).

118. Kaufman, B. et al. Olaparib monotherapy in patients with advanced cancer and a germline BRCA1/2 mutation. J. Clin. Oncol. 33, 244-250 (2015).

119. Domchek, S. M. et al. Efficacy and safety of olaparib monotherapy in germline BRCA1/2 mutation carriers with advanced ovarian cancer and three or more lines of prior therapy. Gynecol. Oncol. 140, 199-203 (2016).

120. Pujade-Lauraine, E. et al. Olaparib tablets as maintenance therapy in patients with platinum-sensitive, relapsed ovarian cancer and a BRCA1/2 mutation (SOLO2/ENGOT-Ov21): a double-blind, randomised, placebo-controlled, phase 3 trial. Lancet Oncol. 18, 1274-1284 (2017).

121. Moore, K. et al. Maintenance olaparib in patients with newly diagnosed advanced ovarian cancer. N. Engl. J. Med. 379, 2495-2505 (2018).

122. Ray-Coquard, I. et al. Olaparib plus bevacizumab as first-line maintenance in ovarian cancer. N. Engl. J. Med. 381, 2416-2428 (2019).

123. Liu, J. F. et al. A Phase 1 trial of the poly(ADP-ribose) polymerase inhibitor olaparib (AZD2281) in combination with the anti-angiogenic cediranib (AZD2171) in recurrent epithelial ovarian or triple-negative breast cancer. Eur. J. Cancer 49, 2972-2978 (2013).

124. Liu, J. F. et al. Combination cediranib and olaparib versus olaparib alone for women with recurrent platinum-sensitive ovarian cancer: a randomised phase 2 study. Lancet Oncol. 15, 1207-1214 (2014).

125. Karakashev, S. et al. BET bromodomain inhibition synergizes with PARP inhibitor in epithelial ovarian cancer. Cell Rep. 21, 3398-3405 (2017).

126. Wang, D. et al. Combined inhibition of PI3K and PARP is effective in the treatment of ovarian cancer cells with wild-type PIK3CA genes. Gynecol. Oncol. 142, 548-556 (2016). 
127. Liu, J. et al. A phase 1 study optimizing the dosing of olaparib tablet formulation combined with cediranib in recurrent ovarian cancer. J. Clin. Oncol. 33, 5559-5559 (2015).

128. Liu, J. F. et al. Overall survival and updated progression-free survival outcomes in a randomized phase II study of combination cediranib and olaparib versus olaparib in relapsed platinum-sensitive ovarian cancer. Ann. Oncol. 30, 551-557 (2019).

129. Gockley, A. A. et al. Durable response in a woman with recurrent low-grade endometrioid endometrial cancer and a germline BRCA2 mutation treated with a PARP inhibitor. Gynecol. Oncol. 150, 219-226 (2018).

130. Wilson, R. H. et al. A phase I study of intravenous and oral rucaparib in combination with chemotherapy in patients with advanced solid tumours. Br. J. Cancer 116, 884-892 (2017).

131. Kondrashova, O. et al. Secondary somatic mutations restoring RAD51C and RAD51D associated with acquired resistance to the PARP inhibitor rucaparib in high-grade ovarian carcinoma. Cancer Discov. 7, 984-998 (2017).

132. Drew, Y. et al. Phase 2 multicentre trial investigating intermittent and continuous dosing schedules of the poly(ADP-ribose) polymerase inhibitor rucaparib in germline BRCA mutation carriers with advanced ovarian and breast cancer. Br. J. Cancer 114, 723-730 (2016).

133. Swisher, E. M. et al. Rucaparib in relapsed, platinum-sensitive high-grade ovarian carcinoma (ARIEL2 Part 1): an international, multicentre, open-label, phase 2 trial. Lancet Oncol. 18, 75-87 (2017).

134. Kristeleit, R. et al. A phase I-II study of the oral PARP inhibitor rucaparib in patients with germline BRCA1/2-mutated ovarian carcinoma or other solid tumors. Clin. Cancer Res. 23, 4095-4106 (2017).

135. Oza, A. M. et al. Antitumor activity and safety of the PARP inhibitor rucaparib in patients with high-grade ovarian carcinoma and a germline or somatic BRCA1 or BRCA2 mutation: integrated analysis of data from Study 10 and ARIEL2. Gynecol. Oncol. 147, 267-275 (2017).

136. Coleman, R. L. et al. Rucaparib maintenance treatment for recurrent ovarian carcinoma after response to platinum therapy (ARIEL3): a randomised, doubleblind, placebo-controlled, phase 3 trial. Lancet 390, 1949-1961 (2017).

137. Sandhu, S. K. et al. The poly(ADP-ribose) polymerase inhibitor niraparib (MK4827) in BRCA mutation carriers and patients with sporadic cancer: a phase 1 dose-escalation trial. Lancet Oncol. 14, 882-892 (2013).

138. Mirza, M. R. et al. Niraparib maintenance therapy in platinum-sensitive, recurrent ovarian cancer. N. Engl. J. Med. 375, 2154-2164 (2016).

139. Fabbro, M. et al. Efficacy and safety of niraparib as maintenance treatment in older patients ( $\geq 70$ years) with recurrent ovarian cancer: Results from the ENGOT-OV16/NOVA trial. Gynecol. Oncol. 152, 560-567 (2019).

140. González-Martín, A. et al. Niraparib in patients with newly diagnosed advanced ovarian cancer. N. Engl. J. Med. 381, 2391-2402 (2019).

141. Moore, K. N. et al. Niraparib monotherapy for late-line treatment of ovarian cancer (QUADRA): a multicentre, open-label, single-arm, phase 2 trial. Lancet Oncol. 20, 636-648 (2019).

142. Mirza, M. R. et al. Niraparib plus bevacizumab versus niraparib alone for platinum-sensitive recurrent ovarian cancer (NSGO-AVANOVA2/ENGOT-ov24): a randomised, phase 2, superiority trial. Lancet Oncol. 20, 1409-1419 (2019).

143. Konstantinopoulos, P. A. et al. Single-arm phases 1 and 2 trial of niraparib in combination with pembrolizumab in patients with recurrent platinum-resistant ovarian carcinoma. JAMA Oncol. 5, 1141-1149 (2019).

144. Oza, A. M. et al. Quality of life in patients with recurrent ovarian cancer treated with niraparib versus placebo (ENGOT-OV16/NOVA): results from a double-blind, phase 3, randomised controlled trial. Lancet Oncol. 19, 1117-1125 (2018).

145. Burki, T. K. Veliparib for advanced ovarian cancer. Lancet Oncol. 20, e616 (2019).

146. Coleman, R. L. et al. A phase II evaluation of the potent, highly selective PARP inhibitor veliparib in the treatment of persistent or recurrent epithelial ovarian, fallopian tube, or primary peritoneal cancer in patients who carry a germline BRCA1 or BRCA2 mutation - an NRG Oncology/Gynecologic Oncology Group study. Gynecol. Oncol. 137, 386-391 (2015).

147. Nishio, S. et al. Phase 1 study of veliparib with carboplatin and weekly paclitaxel in Japanese patients with newly diagnosed ovarian cancer. Cancer Sci. 108, 2213-2220 (2017).

148. Nishikawa, T. et al. Phase 1 dose-escalation study of single-agent veliparib in Japanese patients with advanced solid tumors. Cancer Sci. 108, 1834-1842 (2017).

149. Reiss, K. A. et al. A Phase I study of veliparib (ABT-888) in combination with lowdose fractionated whole abdominal radiation therapy in patients with advanced solid malignancies and peritoneal carcinomatosis. Clin. Cancer Res. 21, 68-76 (2015).

150. Coleman, R. L. et al. Veliparib with first-line chemotherapy and as maintenance therapy in ovarian cancer. N. Engl. J. Med. 381, 2403-2415 (2019).

151. Kunos, C. et al. A phase I-II evaluation of veliparib (NSC \#737664), topotecan, and filgrastim or pegfilgrastim in the treatment of persistent or recurrent carcinoma of the uterine cervix: an NRG Oncology/Gynecologic Oncology Group study. Int. J. Gynecol. Cancer 25, 484-492 (2015).

152. Thaker, P. H. et al. A phase I trial of paclitaxel, cisplatin, and veliparib in the treatment of persistent or recurrent carcinoma of the cervix: an NRG Oncology Study (NCT\#01281852). Ann. Oncol. 28, 505-511 (2017).

153. Dhawan, M. S. et al. Differential toxicity in patients with and without DNA repair mutations: phase $\mathrm{i}$ study of carboplatin and talazoparib in advanced solid tumors. Clin. Cancer Res. 23, 6400-6410 (2017).

154. de Bono, J. et al. Phase I, dose-escalation, two-part trial of the PARP Inhibitor talazoparib in patients with advanced germline BRCA1/2 mutations and selected sporadic cancers. Cancer Discov 7, 620-629 (2017).

155. Lickliter, J. D. et al. A phase I dose-escalation study of BGB-290, a novel PARP1/ 2 selective inhibitor in patients with advanced solid tumors. Asco Meeting. (2016).

156. Friedlander, M. et al. Pamiparib in combination with tislelizumab in patients with advanced solid tumours: results from the dose-escalation stage of a multicentre, open-label, phase 1a/b trial. Lancet Oncol. 20, 1306-1315 (2019).

157. Han, Y. et al. Synergism of PARP inhibitor fluzoparib (HS10160) and MET inhibitor HS10241 in breast and ovarian cancer cells. Am. J. Cancer Res. 9, 608-618 (2019).

158. Wang, L. et al. Pharmacologic characterization of fluzoparib, a novel poly(ADPribose) polymerase inhibitor undergoing clinical trials. Cancer Sci. 110, 1064-1075 (2019).

159. Wang, L., Wang, Q., Xu, Y. \& Han, L. Advances in the treatment of ovarian cancer using PARP inhibitors and the underlying mechanism of resistance. Curr. Drug Targets 21, 167-178 (2019).

160. Kubalanza, K. \& Konecny, G. E. Mechanisms of PARP inhibitor resistance in ovarian cancer. Curr. Opin. Obstet. Gynecol. 32, 36-41 (2020).

161. Labidi-Galy, S. I. et al. Clinical factors associated with prolonged response and survival under olaparib as maintenance therapy in BRCA mutated ovarian cancers. Gynecol. Oncol. 155, 262-269 (2019).

162. McCormick, A. et al. Ovarian cancers harbor defects in nonhomologous end joining resulting in resistance to rucaparib. Clin. Cancer Res. 23, 2050-2060 (2017)

163. Higuchi, T. et al. CTLA-4 blockade synergizes therapeutically with PARP inhibition in BRCA1-deficient ovarian cancer. Cancer Immunol. Res. 3, 1257-1268 (2015).

164. Philip, C. A. et al. Inhibition of PI3K-AKT-mTOR pathway sensitizes endometrial cancer cell lines to PARP inhibitors. BMC Cancer 17, 638 (2017).

165. Katso, R. et al. Cellular function of phosphoinositide 3-kinases: implications for development, homeostasis, and cancer. Annu. Rev. Cell Dev. Biol. 17, 615-675 (2001).

166. Fruman, D. A. \& Rommel, C. PI3K and cancer: lessons, challenges and opportunities. Nat. Rev. Drug Discov. 13, 140 (2014).

167. Wullschleger, S., Loewith, R. \& Hall, M. N. J. C. TOR signaling in growth and metabolism. Cell 124, 471-484 (2006).

168. Huang, K. \& Fingar, D. C. Growing knowledge of the mTOR signaling network. Semin. Cell. Dev. Bio 36, 79-90 (2014).

169. Cheaib, B., Auguste, A. \& Leary, A. The PI3K/Akt/mTOR pathway in ovarian cancer:therapeutic opportunities and challenges. Chinese J. Cancer 34, 4-16 (2015).

170. Papa, A. et al. Cancer-associated PTEN mutants act in a dominant-negative manner to suppress PTEN protein function. Cell 157, 595-610 (2014).

171. Slomovitz, B. M. \& Coleman, R. L. The PI3K/AKT/mTOR pathway as a therapeutic target in endometrial cancer. Clin. Cancer Res. 18, 5856-5864 (2012).

172. Guo, H. et al. The PI3K/AKT pathway and renal cell carcinoma. J. Genet. Genom. 42, 343-353 (2015)

173. Aziz, S. A. et al. Phosphatidylinositol-3-kinase as a therapeutic target in melanoma. Clin. Cancer Res. 15, 3029-3036 (2009).

174. Pivot, X. et al. 6 months versus 12 months of adjuvant trastuzumab in early breast cancer (PHARE): final analysis of a multicentre, open-label, phase 3 randomised trial. Lancet 393, 2591-2598 (2019).

175. Seiji, M., Hiromasa, K., Ryoko, T. \& Tomoyuki, S. J. G. O. The PI3K/AKT/mTOR pathway as a therapeutic target in ovarian cancer. Gynecol. Oncol. 137, 173-179 (2015).

176. Bugide, S. et al. HPIP promotes epithelial-mesenchymal transition and cisplatin resistance in ovarian cancer cells through PI3K/AKT pathway activation. Cell. Oncol. 40, 133-144 (2017).

177. Ke, Z., Caiping, S., Qing, Z. \& Xiaojing, W. Sonic hedgehog-Gli1 signals promote epithelial-mesenchymal transition in ovarian cancer by mediating PI3K/AKT pathway. Med. Oncol. 32, 368 (2015).

178. Mazloumi Gavgani, F. et al. Class I phosphoinositide 3-kinase PIK3CA/p110alpha and PIK3CB/p110beta isoforms in endometrial cancer. Int. J. Mol. Sci. 19, 3931 (2018).

179. Bertelsen, B. I. et al. Molecular analysis of the PI3K-AKT pathway in uterine cervical neoplasia: frequent PIK3CA amplification and AKT phosphorylation. Int. J. Cancer 118, 1877-1883 (2006). 
Targeted therapies in gynecological cancers: a comprehensive review of... Wang et al.

180. Kent, C. N. \& Guttilla Reed, I. K. Regulation of epithelial-mesenchymal transition in endometrial cancer: connecting $\mathrm{PI} 3 \mathrm{~K}$, estrogen signaling, and microRNAs. Clin. Transl. Oncol. 18, 1056-1061 (2016).

181. Oh, K. J., Kalinina, A., Park, N. H. \& Bagchi, S. Deregulation of elF4E: 4E-BP1 in differentiated human papillomavirus-containing cells leads to high levels of expression of the E7 oncoprotein. J. Virol. 80, 7079-7088 (2006).

182. Zhang, D. et al. Long non-coding RNA ANRIL indicates a poor prognosis of cervical cancer and promotes carcinogenesis via PI3K/Akt pathways. Biomed. Pharmacother. 85, 511-516 (2017).

183. Prasad, S. B. et al. PI3K/AKT pathway-mediated regulation of p27(Kip1) is associated with cell cycle arrest and apoptosis in cervical cancer. Cell. Oncol. 38, 215-225 (2015).

184. Weigelt, B. et al. PI3K pathway dependencies in endometrioid endometrial cancer cell lines. Clin. Cancer Res. 19, 3533-3544 (2013).

185. El-Kott, A. F., Shati, A. A., Al-Kahtani, M. A. \& Alqahtani, S. Acylated ghrelin renders chemosensitive ovarian cancer cells resistant to cisplatin chemotherapy via activation of the PI3K/Akt/mTOR survival pathway. Anal. Cell. Pathol. 2019, 9627810 (2019)

186. Schrauwen, S. et al. Dual blockade of PI3K/AKT/mTOR (NVP-BEZ235) and Ras/ Raf/MEK (AZD6244) pathways synergistically inhibit growth of primary endometrioid endometrial carcinoma cultures, whereas NVP-BEZ235 reduces tumor growth in the corresponding xenograft models. Gynecol. Oncol. 138, 165-173 (2015).

187. Fleming, G. F. et al. Temsirolimus with or without megestrol acetate and tamoxifen for endometrial cancer: a gynecologic oncology group study. Gynecol. Oncol. 132, 585-592 (2014).

188. Oza, A. M. et al. Molecular correlates associated with a phase II study of temsirolimus (CCl-779) in patients with metastatic or recurrent endometrial cancerNCIC IND 160. J. Clin. Oncol. 24, 3003-3003 (2006).

189. Santacana, M. et al. Biological effects of temsirolimus on the mTOR pathway in endometrial carcinoma: a Pharmacodynamic Phase II Study. Int. J. Gynecol. Cancer (2016). https://doi.org/10.1097/IGC.0000000000000715. Online ahead of print.

190. Chon, H. S. et al. Phase I study of oral ridaforolimus in combination with paclitaxel and carboplatin in patients with solid tumor cancers. BMC Cancer 17, 407 (2017).

191. Tsoref, D. et al. Phase II study of oral ridaforolimus in women with recurrent or metastatic endometrial cancer. Gynecol. Oncol. 135, 184-189 (2014).

192. Slomovitz, B. M. et al. A phase 2 study of the oral mammalian target of rapamycin inhibitor, everolimus, in patients with recurrent endometrial carcinoma. Cancer 116, 5415-5419 (2010).

193. Soliman, P. T. et al. Phase II study of everolimus, letrozole, and metformin in women with advanced/recurrent endometrial cancer. J. Clin. Oncol. 34, 5506-5506 (2016)

194. Slomovitz, B. M. et al. Phase II study of everolimus and letrozole in patients with recurrent endometrial carcinoma. J. Clin. Oncol. 33, 930-936 (2015).

195. Chiu, J. W. et al. A phase I trial of ANG1/2-Tie2 inhibitor trebaninib (AMG386) and temsirolimus in advanced solid tumors (PJC008/NCImusical sharp9041). Investig. N. Drugs 34, 104-111 (2016).

196. Taylor, S. E. et al. Phase II study of everolimus (EV) and bevacizumab (BEV) in recurrent ovarian, peritoneal, and fallopian tube cancer. J. Clin. Oncol. 34, 5552-5552 (2016).

197. Aghajanian, C. et al. A phase II study of frontline paclitaxel/carboplatin/bevacizumab, paclitaxel/carboplatin/temsirolimus, or ixabepilone/carboplatin/bevacizumab in advanced/recurrent endometrial cancer. Gynecol. Oncol. 150, 274-281 (2018).

198. Basu, B. et al. Vistusertib (dual m-TORC1/2 inhibitor) in combination with paclitaxel in patients with high-grade serous ovarian and squamous non-smallcell lung cancer. Ann. Oncol. 29, 1918-1925 (2018).

199. Mackay, H. J. et al. Molecular determinants of outcome with mammalian target of rapamycin inhibition in endometrial cancer. Cancer 120, 603-610 (2014).

200. Aghajanian, C. et al. A phase I, open-label, two-stage study to investigate the safety, tolerability, pharmacokinetics, and pharmacodynamics of the oral AKT inhibitor GSK2141795 in patients with solid tumors. Investig. N. Drugs 36 1016-1025 (2018)

201. Winder, A. et al. The allosteric AKT inhibitor, MK2206, decreases tumor growth and invasion in patient derived xenografts of endometrial cancer. Cancer Biol. Ther. 18, 958-964 (2017).

202. Liu, J. F. et al. Results from a single arm, single stage phase II trial of trametinib and GSK2141795 in persistent or recurrent cervical cancer. Gynecol. Oncol. 154 95-101 (2019).

203. Westin, S. N. et al. Limited access safety lead-in of the MEK inhibitor trametinib in combination with GSK2141795, an AKT inhibitor, in patients with recurrent or persistent endometrial cancer: a Gynecologic Oncology Group study. Gynecol. Oncol. 141, 4-5 (2016).
204. Westin, S. N. et al. Safety lead-in of the MEK inhibitor trametinib in combination with GSK2141795, an AKT inhibitor, in patients with recurrent endometrial cancer: an NRG Oncology/GOG study. Gynecol. Oncol. 155, 420-428 (2019).

205. Myers, A. P. et al. Phase II, two-stage, two-arm, PIK3CA mutation stratified trial of MK-2206 in recurrent endometrial cancer (EC). J. Clin. Oncol. 31, 5524-5524 (2013).

206. Blagden, S. P. et al. Phase IB dose escalation and expansion study of AKT inhibitor afuresertib with carboplatin and paclitaxel in recurrent platinumresistant ovarian cancer. Clin. Cancer Res. 25, 1472-1478 (2019).

207. Bendell, J. C. et al. Phase I, dose-escalation study of BKM120, an oral pan-Class I $\mathrm{PI} 3 \mathrm{~K}$ inhibitor, in patients with advanced solid tumors. J. Clin. Oncol. 30, 282-290 (2012).

208. Cao, P. et al. PI3K p110alpha inhibition sensitizes cervical cancer cells with aberrant PI3K signaling activation to PARP inhibitor BMN673. Oncol. Rep. 42, 2097-2107 (2019)

209. Heudel, P. E. et al. Phase II study of the PI3K inhibitor BKM120 in patients with advanced or recurrent endometrial carcinoma: a stratified type I-type II study from the GINECO group. Br. J. Cancer 116, 303-309 (2017).

210. Matulonis, U. et al. Phase II study of the PI3K inhibitor pilaralisib (SAR245408; $\mathrm{XL147)}$ in patients with advanced or recurrent endometrial carcinoma. Gynecol. Oncol. 136, 246-253 (2015).

211. Wainberg, Z. A. et al. A multi-arm phase i study of the PI3K/mTOR inhibitors PF04691502 and gedatolisib (PF-05212384) plus irinotecan or the MEK inhibitor PD-0325901 in advanced cancer. Target Oncol. 12, 775-785 (2017).

212. Del Campo, J. M. et al. A randomized phase II non-comparative study of PF04691502 and gedatolisib (PF-05212384) in patients with recurrent endometrial cancer. Gynecol. Oncol. 142, 62-69 (2016).

213. Roncolato, F. et al. PI3K/AKT/mTOR inhibitors for advanced or recurrent endometrial cancer. Cochrane Database Syst. Rev. 10, Cd012160 (2019).

214. Kyriakopoulos, C. E. et al. A phase I study of tivantinib in combination with temsirolimus in patients with advanced solid tumors. Investig. N. Drugs 35, 290-297 (2017).

215. Normanno, N. et al. Epidermal growth factor receptor (EGFR) signaling in cancer Gene 366, 2-16 (2006).

216. Yarden, Y. \& Sliwkowski, M. X. Untangling the ErbB signalling network. Nat. Rev. Mol. Cell Biol. 2, 127-137 (2001).

217. Zhang, X. et al. An allosteric mechanism for activation of the kinase domain of epidermal growth factor receptor. Cell 125, 1137-1149 (2006).

218. Citri, A., Skaria, K. B. \& Yarden, Y. The deaf and the dumb: the biology of ErbB-2 and ErbB-3. Exp. C ell Res. 284, 54-65 (2003).

219. Christgen, M. et al. Activating human epidermal growth factor receptor 2 (HER2) gene mutation in bone metastases from breast cancer. Virchows Arch. : Int. J. Pathol. 473, 577-582 (2018).

220. Moasser, M. M. The oncogene HER2: its signaling and transforming functions and its role in human cancer pathogenesis. Oncogene 26, 6469-6487 (2007).

221. Lau, T. S. et al. A loop of cancer-stroma-cancer interaction promotes peritoneal metastasis of ovarian cancer via TNFalpha-TGFalpha-EGFR. Oncogene 36, 3576-3587 (2017).

222. Lin, T. C. et al. GALNT6 expression enhances aggressive phenotypes of ovarian cancer cells by regulating EGFR activity. Oncotarget 8, 42588-42601 (2017).

223. Slomovitz, B. M. et al. Her-2/neu overexpression and amplification in uterine papillary serous carcinoma. J. Clin. Oncol. 22, 3126-3132 (2004).

224. Albagoush, S. A. \& Limaiem, F. in StatPearls (StatPearls Publishing StatPearls Publishing LLC., 2019)

225. von Minckwitz, G. et al. Adjuvant pertuzumab and trastuzumab in early HER2positive breast cancer. N. Engl. J. Med. 377, 122-131 (2017).

226. Menderes, G. et al. Efficacy of neratinib in the treatment of HER2/neu-amplified epithelial ovarian carcinoma in vitro and in vivo. Med. Oncol. 34, 91 (2017).

227. Menderes, G. et al. SYD985, a novel duocarmycin-based HER2-targeting antibody-drug conjugate, shows promising antitumor activity in epithelial ovarian carcinoma with HER2/Neu expression. Gynecol. Oncol. 146, 179-186 (2017).

228. Gianni, L. et al. Efficacy and safety of neoadjuvant pertuzumab and trastuzumab in women with locally advanced, inflammatory, or early HER2-positive breast cancer (NeoSphere): a randomised multicentre, open-label, phase 2 trial. Lancet Oncol. 13, 25-32 (2012).

229. Slamon, D. J. et al. Use of chemotherapy plus a monoclonal antibody against HER2 for metastatic breast cancer that overexpresses HER2. N. Engl. J. Med. 344, 783-792 (2001).

230. Hudis, C. A. Trastuzumab-mechanism of action and use in clinical practice. $N$. Engl. J. Med. 357, 39-51 (2007).

231. Agus, D. B. et al. Targeting ligand-activated ErbB2 signaling inhibits breast and prostate tumor growth. Cancer Cell 2, 127-137 (2002).

232. Barok, M., Joensuu, H. \& Isola, J. Trastuzumab emtansine: mechanisms of action and drug resistance. Breast Cancer Res. 16, 209 (2014). 
233. Xia, W. et al. Anti-tumor activity of GW572016: a dual tyrosine kinase inhibitor blocks EGF activation of EGFR/erbB2 and downstream Erk1/2 and AKT pathways. Oncogene 21, 6255-6263 (2002).

234. Rawluk, J. \& Waller, C. F. Gefitinib. Recent Results Cancer Res. 211, 235-246 (2018).

235. Roskoski, R. Jr Small molecule inhibitors targeting the EGFR/ErbB family of protein-tyrosine kinases in human cancers. Pharmacol. Res. 139, 395-411 (2019).

236. Voigtlaender, M., Schneider-Merck, T. \& Trepel, M. Lapatinib. Recent Results Cancer Res. 211, 19-44 (2018).

237. Konecny, G. E. et al. HER2 gene amplification and EGFR expression in a large cohort of surgically staged patients with nonendometrioid (type II) endometrial cancer. Br. J. Cancer 100, 89-95 (2009).

238. Buza, N., English, D. P., Santin, A. D. \& Hui, P. Toward standard HER2 testing of endometrial serous carcinoma: 4 -year experience at a large academic center and recommendations for clinical practice. Mod. Pathol. 26, 1605-1612 (2013).

239. Bookman, M. A. et al. Evaluation of monoclonal humanized anti-HER2 antibody, trastuzumab, in patients with recurrent or refractory ovarian or primary peritoneal carcinoma with overexpression of HER2: a phase II trial of the Gynecologic Oncology Group. J. Clin. Oncol. 21, 283-290 (2003).

240. Melissa, H., Tasha, S. G. \& Tewari, K. S. A review of HER2-targeted therapy in breast and ovarian cancer: lessons from antiquity-CLEOPATRA and PENELOPE. Future Oncol. 11, 3113-3131 (2015).

241. Cousin, S. et al. Targeting ERBB2 mutations in solid tumors: biological and clinical implications. J. Hematol. Oncol. 11, 86 (2018).

242. Hyman, D. M. et al. HER kinase inhibition in patients with HER2- and HER3mutant cancers. Nature 554, 189-194 (2018).

243. Petrelli, F., Borgonovo, K., Cabiddu, M. \& Barni, S. Efficacy of EGFR tyrosine kinase inhibitors in patients with EGFR-mutated non-small-cell lung cancer: a metaanalysis of 13 randomized trials. Clin. Lung cancer 13, 107-114 (2012).

244. Piccirillo, M. C. et al. D01*MITO (Multicentre Italian Trials in Ovarian cancer)CERV 2 trial: a randomized phase II study of carboplatin and paclitaxel +/cetuximab, in advanced and/or recurrent cervical cancer. Ann. Oncol. 26, vi33-vi33 (2015).

245. Farley, J. et al. Phase II study of cisplatin plus cetuximab in advanced, recurrent, and previously treated cancers of the cervix and evaluation of epidermal growth factor receptor immunohistochemical expression: a Gynecologic Oncology Group study. Gynecol. Oncol. 121, 303-308 (2011).

246. Kurtz, J. E. et al. Cetuximab, topotecan and cisplatin for the treatment of advanced cervical cancer: A phase II GINECO trial. Gynecol. Oncol. 113, 16-20 (2009).

247. Pignata, S. et al. The MITO CERV-2 trial: A randomized phase II study of cetuximab plus carboplatin and paclitaxel, in advanced or recurrent cervical cancer. Gynecol. Oncol. 153, 535-540 (2019).

248. Secord, A. A. et al. Phase II trial of cetuximab and carboplatin in relapsed platinum-sensitive ovarian cancer and evaluation of epidermal growth factor receptor expression: a Gynecologic Oncology Group study. Gynecol. Oncol. 108, 493-499 (2008).

249. Fleming, G. F. et al. Phase II trial of trastuzumab in women with advanced or recurrent, HER2-positive endometrial carcinoma: a Gynecologic Oncology Group study. Gynecol. Oncol. 116, 15-20 (2010).

250. Fader, A. N. et al. Randomized phase II trial of carboplatin-paclitaxel versus carboplatin-paclitaxel-trastuzumab in uterine serous carcinomas that overexpress human epidermal growth factor. Receptor 2/neu. 36, 2044-2051 (2018).

251. Yang, Y., Lu, Z.-S. \& Zeng, Z. Clinical efficacy and safety of combination of abraxane and trastuzumab in treatment of recurrent ovarian cancer. Pak. J. Pharm. Sci. 31, 2831-2834 (2018).

252. Makhija, S. et al. Clinical activity of gemcitabine plus pertuzumab in platinumresistant ovarian cancer, fallopian tube cancer, or primary peritoneal cancer. J. Clin. Oncol. 28, 1215-1223 (2010).

253. Lorusso, D. et al. Patient-reported outcomes and final overall survival results from the randomized phase 3 PENELOPE trial evaluating pertuzumab in low tumor human epidermal growth factor receptor 3 (HER3) mRNA-expressing platinum-resistant ovarian cancer. Int. J. Gynecol. Cancer 29, 1141-1147 (2019).

254. Kurzeder, C. et al. Double-blind, placebo-controlled, randomized phase III trial evaluating pertuzumab combined with chemotherapy for low tumor human epidermal growth factor receptor 3 mRNA-expressing platinum-resistant ovarian cancer (PENELOPE). J. Clin. Oncol. 34, 2516-2525 (2016).

255. Kaye, S. B. et al. A randomized phase II study evaluating the combination of carboplatin-based chemotherapy with pertuzumab versus carboplatin-based therapy alone in patients with relapsed, platinum-sensitive ovarian cancer. Ann. Oncol. 24, 145-152 (2013).

256. Leslie, K. K. et al. Lapatinib and potential prognostic value of EGFR mutations in a Gynecologic Oncology Group phase II trial of persistent or recurrent endometrial cancer. Gynecol. Oncol. 127, 345-350 (2012).
257. Monk, B. J. et al. Phase II, open-label study of pazopanib or lapatinib monotherapy compared with pazopanib plus lapatinib combination therapy in patients with advanced and recurrent cervical cancer. J. Clin. Oncol. 28, 3562-3569 (2010).

258. Garcia, A. A. et al. A phase II evaluation of lapatinib in the treatment of persistent or recurrent epithelial ovarian or primary peritoneal carcinoma: a gynecologic oncology group study. Gynecol. Oncol. 124, 569-574 (2012).

259. Lheureux, S. et al. Expected benefits of topotecan combined with lapatinib in recurrent ovarian cancer according to biological profile: a phase 2 trial. Int. J. Gynecol. Cancer 22, 1483-1488 (2012).

260. Weroha, S. J. et al. Phase II trial of lapatinib and topotecan (LapTop) in patients with platinum-refractory/resistant ovarian and primary peritoneal carcinoma. Gynecol. Oncol. 122, 116-120 (2011).

261. Nimeiri, H. S. et al. Efficacy and safety of bevacizumab plus erlotinib for patients with recurrent ovarian, primary peritoneal, and fallopian tube cancer: a trial of the Chicago, PMH, and California Phase II Consortia. Gynecol. Oncol. 110, 49-55 (2008).

262. Vergote, I. B. et al. Randomized phase III study of erlotinib versus observation in patients with no evidence of disease progression after first-line platin-based chemotherapy for ovarian carcinoma: a European Organisation for Research and Treatment of Cancer-Gynaecological Cancer Group, and Gynecologic Cancer Intergroup study. J. Clin. Oncol. 32, 320-326 (2014).

263. Nogueira-Rodrigues, A. et al. Phase 2 trial of erlotinib combined with cisplatin and radiotherapy in patients with locally advanced cervical cancer. Cancer 120, 1187-1193 (2014).

264. Campos, S. et al. Multicenter, randomized phase II trial of oral Cl-1033 for previously treated advanced ovarian cancer. J. Clin. Oncol. 23, 5597-5604 (2005).

265. Annunziata, C. M. et al. Vandetanib, designed to inhibit VEGFR2 and EGFR signaling, had no clinical activity as monotherapy for recurrent ovarian cancer and no detectable modulation of VEGFR2. Clin. Cancer Res. 16, 664-672 (2010).

266. Goncalves, A. et al. A phase II trial to evaluate gefitinib as second- or third-line treatment in patients with recurring locoregionally advanced or metastatic cervical cancer. Gynecol. Oncol. 108, 42-46 (2008).

267. Harter, P. et al. Addition of vandetanib to pegylated liposomal doxorubicin (PLD) in patients with recurrent ovarian cancer. A randomized phase $1 / I$ study of the AGO Study Group (AGO-OVAR 2.13). Investig. N. Drugs 31, 1499-1504 (2013).

268. Sims, A. H. et al. Defining the molecular response to trastuzumab, pertuzumab and combination therapy in ovarian cancer. Br. J. Cancer 106, 1779-1789 (2012).

269. Hassan, W., Chitcholtan, K., Sykes, P. \& Garrill, A. A combination of two receptor tyrosine kinase inhibitors, canertinib and PHA665752 compromises ovarian cancer cell growth in 3D cell models. Oncol. Ther. 4, 257-274 (2016).

270. Su, F. et al. SP1 promotes tumor angiogenesis and invasion by activating VEGF expression in an acquired trastuzumabresistant ovarian cancer model. Oncol. Rep. 38, 2677-2684 (2017).

271. Maekawa, M., Nishida, E. \& Tanoue, T. Identification of the anti-proliferative protein Tob as a MAPK substrate. J. Biol. Chem. 277, 37783-37787 (2002).

272. Malumbres, M. \& Barbacid, M. RAS oncogenes: the first 30 years. Nat. Rev. Cancer 3, 459-465 (2003).

273. Yoon, S. \& Seger, R. The extracellular signal-regulated kinase: multiple substrates regulate diverse cellular functions. Growth Factors 24, 21-44 (2006).

274. Friday, B. B. \& Adjei, A. A. Advances in targeting the Ras/Raf/MEK/Erk mitogenactivated protein kinase cascade with MEK inhibitors for cancer therapy. Clin. Cancer Res. 14, 342-346 (2008).

275. Zhong, F. et al. Comprehensive genomic profiling of high-grade serous ovarian carcinoma from Chinese patients identifies co-occurring mutations in the Ras/ Raf pathway withTP53. Cancer Med. 8, 3928-3935 (2019).

276. Xu, D. et al. Raf-ERK1/2 signalling pathways mediate steroid hormone synthesis in bovine ovarian granulosa cells. Reprod. Domest. Anim. = Zuchthyg. 54, 741-749 (2019).

277. Moorthy, N. S., Sousa, S. F., Ramos, M. J. \& Fernandes, P. A. Farnesyltransferase inhibitors: a comprehensive review based on quantitative structural analysis. Curr. Medicinal Chem. 20, 4888-4923 (2013).

278. Meier, W. et al. Randomized phase II trial of carboplatin and paclitaxel with or without lonafarnib in first-line treatment of epithelial ovarian cancer stage IIB-IV. Gynecol. Oncol. 126, 236-240 (2012).

279. Wilhelm, S. et al. Discovery and development of sorafenib: a multikinase inhibitor for treating cancer. Nat. Rev. Drug Discov. 5, 835-844 (2006).

280. Ganesan, P. et al. Phase I clinical trial of lenalidomide in combination with sorafenib in patients with advanced cancer. Investig. N. Drugs 32, 279-286 (2014).

281. Smolle, E., Taucher, V., Petru, E. \& Haybaeck, J. Targeted treatment of ovarian cancer-the multiple-kinase-inhibitor sorafenib as a potential option. Anticancer Res. 34, 1519-1530 (2014).

282. Siu, L. L. et al. Phase I trial of sorafenib and gemcitabine in advanced solid tumors with an expanded cohort in advanced pancreatic cancer. Clin. Cancer Res. 12, 144-151 (2006). 
Targeted therapies in gynecological cancers: a comprehensive review of... Wang et al.

283. Trump, D. L. J. U. O. S. \& \& Investigations, O. 1. Sorafenib in advanced clear-cell renal-cell carcinoma. N. Engl. J. Med. 25, 443-445 (2007).

284. Haas, N. B. et al. Adjuvant sunitinib or sorafenib for high-risk, non-metastatic renal-cell carcinoma (ECOG-ACRIN E2805): a double-blind, placebo-controlled, randomised, phase 3 trial. Lancet 387, 2008-2016 (2016).

285. Kudo, M. et al. Sorafenib plus low-dose cisplatin and fluorouracil hepatic arteria infusion chemotherapy versus sorafenib alone in patients with advanced hepatocellular carcinoma (SILIUS): a randomised, open label, phase 3 trial. Lancet Gastroenterol. Hepatol. 3, 424-432 (2018).

286. Bodnar, L., Gornas, M. \& Szczylik, C. Sorafenib as a third line therapy in patients with epithelial ovarian cancer or primary peritoneal cancer: a phase II study. Gynecol. Oncol. 123, 33-36 (2011).

287. Park, G. B., Ko, H. S. \& Kim, D. Sorafenib controls the epithelialmesenchymal transition of ovarian cancer cells via EGF and the CD44HA signaling pathway in a cell typedependent manner. Mol. Med. Rep. 16, 1826-1836 (2017).

288. Hainsworth, J. D. et al. Paclitaxel/carboplatin with or without sorafenib in the first-line treatment of patients with stage III/IV epithelial ovarian cancer: a randomized phase II study of the Sarah Cannon Research Institute. Cancer Med 4 673-681 (2015).

289. Herzog, T. J. et al. A randomized phase II trial of maintenance therapy with Sorafenib in front-line ovarian carcinoma. Gynecol. Oncol. 130, 25-30 (2013).

290. Chekerov, R. et al. Sorafenib plus topotecan versus placebo plus topotecan for platinum-resistant ovarian cancer (TRIAS): a multicentre, randomised, doubleblind, placebo-controlled, phase 2 trial. Lancet Oncol. 19, 1247-1258 (2018).

291. Schwandt, A. et al. Randomized phase II trial of sorafenib alone or in combination with carboplatin/paclitaxel in women with recurrent platinum sensitive epithelial ovarian, peritoneal, or fallopian tube cancer. Investig. N. Drugs 32, 729-738 (2014).

292. Milosevic, M. F. et al. Sorafenib increases tumor hypoxia in cervical cancer patients treated with radiation therapy: results of a phase 1 clinical study. Int J. Radiat. Oncol. Biol. Phys. 94, 111-117 (2016).

293. Ho, A. L. et al. Selumetinib-enhanced radioiodine uptake in advanced thyroid cancer. N. Engl. J. Med. 368, 623-632 (2013).

294. Janne, P. A. et al. Selumetinib plus docetaxel for KRAS-mutant advanced nonsmall-cell lung cancer: a randomised, multicentre, placebo-controlled, phase 2 study. Lancet Oncol. 14, 38-47 (2013).

295. Chiu, H. C. et al. Epithelial to mesenchymal transition and cell biology of molecular regulation in endometrial carcinogenesis. J. Clin. Med. 8, 439 (2019).

296. Ring, K. L. et al. Endometrial cancers with activating kras mutations have activated estrogen signaling and paradoxical response to MEK inhibition. Int. J. Gynecol. Cancer 27, 854-862 (2017).

297. Coleman, R. L. et al. A phase II evaluation of selumetinib (AZD6244, ARRY142886), a selective MEK-1/2 inhibitor in the treatment of recurrent or persistent endometrial cancer: an NRG Oncology/Gynecologic Oncology Group study. Gynecol. Oncol. 138, 30-35 (2015).

298. Farley, J. et al. Selumetinib in women with recurrent low-grade serous carcinoma of the ovary or peritoneum: an open-label, single-arm, phase 2 study. Lancet Oncol. 14, 134-140 (2013).

299. Bedard, P. L. et al. A phase lb dose-escalation study of the oral pan-PI3K inhibitor buparlisib (BKM120) in combination with the oral MEK1/2 inhibitor trametinib (GSK1120212) in patients with selected advanced solid tumors. Clin. Cancer Res. 21, 730-738 (2015).

300. Groner, B. \& von Manstein, V. Jak Stat signaling and cancer: opportunities, benefits and side effects of targeted inhibition. Mol. Cell. Endocrinol. 451, 1-14 (2017).

301. Borges, S. et al. Involvement of a JAK/STAT pathway inhibitor: cytokine inducible $\mathrm{SH} 2$ containing protein in breast cancer. Adv. Exp. Med. Biol. 617, 321-329 (2008).

302. Slattery, M. L. et al. JAK/STAT/SOCS-signaling pathway and colon and rectal cancer. Mol. Carcinog. 52, 155-166 (2013).

303. Passamonti, F. et al. Ruxolitinib for the treatment of inadequately controlled polycythaemia vera without splenomegaly (RESPONSE-2): a randomised, openlabel, phase 3b study. Lancet Oncol. 18, 88-99 (2017).

304. Shang, A. Q. et al. Relationship between HER2 and JAK/STAT-SOCS3 signaling pathway and clinicopathological features and prognosis of ovarian cancer. Cancer Biol. Ther. 18, 314-322 (2017).

305. Ruan, Z., Yang, X. \& Cheng, W. OCT4 accelerates tumorigenesis through activating JAK/STAT signaling in ovarian cancer side population cells. Cancer Manag. Res. 11, 389-399 (2019).

306. Han, E. S. et al. Therapeutic potential of ruxolitinib in human ovarian cancer. Gynecol. Oncol. 141, 45-46 (2016).

307. Wen, W. et al. Increasing antitumor activity of JAK inhibitor by simultaneous blocking multiple survival signaling pathways in human ovarian cancer. Transl. Oncol. 12, 1015-1025 (2019).

308. Basilico, C. et al. A high affinity hepatocyte growth factor-binding site in the immunoglobulin-like region of Met. J. Biol. Chem. 283, 21267-21277 (2008).
309. Trusolino, L., Bertotti, A. \& Comoglio, P. M. MET signalling: principles and functions in development, organ regeneration and cancer. Nat. Rev. Mol. Cell Biol. 11, 834-848 (2010)

310. Gallo, S., Sala, V., Gatti, S. \& Crepaldi, T. Cellular and molecular mechanisms of HGF/Met in the cardiovascular system. Clin. Sci. 129, 1173-1193 (2015).

311. Schmidt, L. et al. Germline and somatic mutations in the tyrosine kinase domain of the MET proto-oncogene in papillary renal carcinomas. Nat. Genet. 16, 68-73 (1997).

312. Lutterbach, B. et al. Lung cancer cell lines harboring MET gene amplification are dependent on Met for growth and survival. Cancer Res. 67, 2081-2088 (2007).

313. Boccaccio, C. \& Comoglio, P. M. Invasive growth: a MET-driven genetic programme for cancer and stem cells. Nat. Rev. Cancer 6, 637-645 (2006).

314. Kwon, Y. et al. Effective inhibition of c-MET-mediated signaling, growth and migration of ovarian cancer cells is influenced by the ovarian tissue microenvironment. Oncogene 34, 144-153 (2015).

315. Han, Z. et al. Alpha-ketobenzothiazole serine protease inhibitors of aberrant HGF/c-MET and MSP/RON kinase pathway signaling in cancer. ChemMedChem 11, 585-599 (2016).

316. Yamamoto, S. et al. Gene amplification and protein overexpression of MET are common events in ovarian clear-cell adenocarcinoma: their roles in tumor progression and prognostication of the patient. Mod. Pathol. 24, 1146-1155 (2011).

317. Baykal, C. et al. Overexpression of the c-Met/HGF receptor and its prognostic significance in uterine cervix carcinomas. Gynecol. Oncol. 88, 123-129 (2003).

318. Jardim, D. L. et al. Analysis of 1,115 patients tested for MET amplification and therapy response in the MD Anderson Phase I Clinic. Clin. Cancer Res. 20, 6336-6345 (2014)

319. Yamashita, Y. et al. Met is the most frequently amplified gene in endometriosisassociated ovarian clear cell adenocarcinoma and correlates with worsened prognosis. PLoS ONE 8, e57724 (2013).

320. Wang, $\mathrm{H}$. et al. Expression and significance of CD44, CD47 and c-met in ovarian clear cell carcinoma. Int. J. Mol. Sci. 16, 3391-3404 (2015).

321. Li, M. et al. HGF and c-Met in pathogenesis of endometrial carcinoma. Front. Biosci. 20, 635-643 (2015).

322. Martin, L. P. et al. A phase II evaluation of AMG 102 (rilotumumab) in the treatment of persistent or recurrent epithelial ovarian, fallopian tube or primary peritoneal carcinoma: a Gynecologic Oncology Group study. Gynecol. Oncol. 132, 526-530 (2014).

323. Konstantinopoulos, P. A. et al. Phase II study of single-agent cabozantinib in patients with recurrent clear cell ovarian, primary peritoneal or fallopian tube cancer (NRG-GY001). Gynecol. Oncol. 150, 9-13 (2018).

324. Matulonis, U. A. et al. A randomized phase II study of cabozantinib versus weekly paclitaxel in the treatment of persistent or recurrent epithelial ovarian, fallopian tube or primary peritoneal cancer: An NRG Oncology/Gynecologic Oncology Group study. Gynecol. Oncol. 152, 548-553 (2019).

325. Yeatman, T. J. A renaissance for SRC. Nat. Rev. Cancer 4, 470-480 (2004).

326. Pengetnze, Y. et al. Src tyrosine kinase promotes survival and resistance to chemotherapeutics in a mouse ovarian cancer cell line. Biochem. Biophys. Res. Commun. 309, 377-383 (2003).

327. Duxbury, M. S. et al. siRNA directed against c-Src enhances pancreatic adenocarcinoma cell gemcitabine chemosensitivity. J. Am. Coll. Surg. 198, 953-959 (2004).

328. Le, X. F. \& Bast, R. C. Jr Src family kinases and paclitaxel sensitivity. Cancer Biol. Ther. 12, 260-269 (2011).

329. Chen, T., Pengetnze, Y. \& Taylor, C. C. Src inhibition enhances paclitaxel cytotoxicity in ovarian cancer cells by caspase-9-independent activation of caspase3. Mol. Cancer Ther. 4, 217-224 (2005).

330. Simpkins, F. et al. Dual Src and MEK inhibition decreases ovarian cancer growth and targets tumor initiating stem-like cells. Clin. Cancer Res. 24, 4874-4886 (2018).

331. Schilder, R. J. et al. Phase II evaluation of dasatinib in the treatment of recurrent or persistent epithelial ovarian or primary peritoneal carcinoma: a Gynecologic Oncology Group study. Gynecol. Oncol. 127, 70-74 (2012).

332. McNeish, I. A. et al. A randomised, placebo-controlled trial of weekly paclitaxel and saracatinib (AZD0530) in platinum-resistant ovarian, fallopian tube or primary peritoneal cancerdagger. Ann. Oncol. 25, 1988-1995 (2014).

333. Takebe, N. et al. Targeting Notch, Hedgehog, and Wnt pathways in cancer stem cells: clinical update. Nat. Rev. Clin. Oncol. 12, 445-464 (2015).

334. Gomez-del Arco, P. et al. Alternative promoter usage at the Notch1 locus supports ligand-independent signaling in T cell development and leukemogenesis. Immunity 33, 685-698 (2010).

335. Zhou, J. et al. Notch and TGFbeta form a positive regulatory loop and regulate EMT in epithelial ovarian cancer cells. Cell. Signal. 28, 838-849 (2016).

336. Mamaeva, V. et al. Inhibiting notch activity in breast cancer stem cells by glucose functionalized nanoparticles carrying gamma-secretase inhibitors. Mol. Ther. : J. Am. Soc. Gene Ther. 24, 926-936 (2016). 
337. Shang, C., Lang, B. \& Meng, L. R. Blocking NOTCH pathway can enhance the effect of EGFR inhibitor through targeting CD133+ endometrial cancer cells. Cancer Biol. Ther. 19, 113-119 (2018).

338. Chen, C. et al. Prognostic roles of Notch receptor mRNA expression in human ovarian cancer. Oncotarget 8, 32731-32740 (2017).

339. Diaz-Padilla, I. et al. A phase II study of single-agent RO4929097, a gammasecretase inhibitor of Notch signaling, in patients with recurrent platinumresistant epithelial ovarian cancer: a study of the Princess Margaret, Chicago and California phase II consortia. Gynecol. Oncol. 137, 216-222 (2015).

340. Qiu, Z., Oleinick, N. L. \& Zhang, J. ATR/CHK1 inhibitors and cancer therapy. Radiother. Oncol. : J. Eur. Soc. Ther. Radiol. Oncol. 126, 450-464 (2018).

341. Leijen, S. et al. Phase II study of WEE1 inhibitor AZD1775 plus carboplatin in patients with TP53-mutated ovarian cancer refractory or resistant to first-line therapy within 3 months. J. Clin. Oncol. 34, 4354-4361 (2016).

342. Thomas, A., Teicher, B. A. \& Hassan, R. Antibody-drug conjugates for cancer therapy. Lancet Oncol. 17, e254-e262 (2016).

343. Richardson, D. L., Seward, S. M. \& Moore, K. N. Antibody drug conjugates in the treatment of epithelial ovarian cancer. Hematol./Oncol. Clin. North Am. 32, 1057-1071 (2018).

344. Lee, E. K. \& Liu, J. F. Antibody-drug conjugates in gynecologic malignancies. Gynecol. Oncol. 153, 694-702 (2019).

345. Moore, K. N. et al. A review of mirvetuximab soravtansine in the treatment of platinum-resistant ovarian cancer. Future Oncol. 14, 123-136 (2018).

346. Moore, K. N. et al. Safety and activity findings from a phase $1 \mathrm{~b}$ escalation study of mirvetuximab soravtansine, a folate receptor alpha (FRalpha)-targeting antibody-drug conjugate (ADC), in combination with carboplatin in patients with platinum-sensitive ovarian cancer. Gynecol. Oncol. 151, 46-52 (2018).

347. Moore, K. N. et al. Phase 1 dose-escalation study of mirvetuximab soravtansine (IMGN853), a folate receptor alpha-targeting antibody-drug conjugate, in patients with solid tumors. Cancer 123, 3080-3087 (2017).

348. Ab, O. et al. IMGN853, a folate receptor-alpha (FRalpha)-targeting antibody-drug conjugate, exhibits potent targeted antitumor activity against FRalphaexpressing tumors. Mol. Cancer Ther. 14, 1605-1613 (2015).

349. Cheung, A. et al. Targeting folate receptor alpha for cancer treatment. Oncotarget 7, 52553-52574 (2016).

350. Ponte, J. F. et al. Mirvetuximab soravtansine (IMGN853), a folate receptor alpha-targeting antibody-drug conjugate, potentiates the activity of standard of care therapeutics in ovarian cancer models. Neoplasia 18, 775-784 (2016).

351. Moore, K. N. et al. Safety and activity of mirvetuximab soravtansine (IMGN853), a folate receptor alpha-targeting antibody-drug conjugate, in platinum-resistant ovarian, fallopian tube, or primary peritoneal cancer: a Phase I Expansion Study. J. Clin. Oncol. 35, 1112-1118 (2017).

352. Moore, K. N. et al. Safety and activity of mirvetuximab soravtansine (IMGN853), a folate receptor alpha-targeting antibody-drug conjugate, in platinum-resistant ovarian, fallopian tube, or primary peritoneal cancer: a Phase I Expansion Study. J. Clin. Oncol. 35, 1112-1118 (2017).

353. Wolford, J. E. \& Tewari, K. S. Rational design for cervical cancer therapeutics: cellular and non-cellular based strategies on the horizon for recurrent, metastatic or refractory cervical cancer. Expert Opin. Drug Discov. 13, 445-457 (2018).

354. de Bono, J. S. et al. Tisotumab vedotin in patients with advanced or metastatic solid tumours (InnovaTV 201): a first-in-human, multicentre, phase 1-2 trial. Lancet Oncol. 20, 383-393 (2019).

355. Okazaki, T. \& Honjo, T. PD-1 and PD-1 ligands: from discovery to clinical application. Int. Immunol. 19, 813-824 (2007).

356. Postow, M. A., Callahan, M. K. \& Wolchok, J. D. Immune checkpoint blockade in cancer therapy. J. Clin. Oncol. 33, 1974-1982 (2015).

357. Herbst, R. S. et al. Predictive correlates of response to the anti-PD-L1 antibody MPDL3280A in cancer patients. Nature 515, 563, (2014).

358. Le, D. T. et al. Mismatch repair deficiency predicts response of solid tumors to PD-1 blockade. Science 357, 409-413 (2017).

359. Mok, T. S. K. et al. Pembrolizumab versus chemotherapy for previously untreated, PD-L1-expressing, locally advanced or metastatic non-small-cell lung cancer (KEYNOTE-042): a randomised, open-label, controlled, phase 3 trial. Lancet 393, 1819-1830 (2019).

360. Rini, B. I. et al. Pembrolizumab plus axitinib versus sunitinib for advanced renalcell carcinoma. N. Engl. J. Med. 380, 1116-1127 (2019).

361. Hodi, F. S. et al. Nivolumab plus ipilimumab or nivolumab alone versus ipilimumab alone in advanced melanoma (CheckMate 067): 4-year outcomes of a multicentre, randomised, phase 3 trial. Lancet Oncol. 19, 1480-1492 (2018).

362. Buderath, P. et al. Prognostic significance of PD-1 and PD-L1 positive tumorinfiltrating immune cells in ovarian carcinoma. Int. J. Gynecol. Cancer 29, 1389-1395 (2019).

363. Pawlowska, A. et al. Immunotherapies based on PD-1/PD-L1 pathway inhibitors in ovarian cancer treatment. Clin. Exp. Immunol. 195, 334-344 (2019).
364. Tan, D., Sheng, L. \& Yi, Q. H. Correlation of PD-1/PD-L1 polymorphisms and expressions with clinicopathologic features and prognosis of ovarian cancer. Cancer Biomark. : Sect. A Dis. Markers 21, 287-297 (2018).

365. Chung, H. C. et al. Efficacy and safety of pembrolizumab in previously treated advanced cervical cancer: results from the phase II KEYNOTE-158 study. J. Clin. Oncol. 37, 1470-1478 (2019).

366. Makker, V. et al. Lenvatinib plus pembrolizumab in patients with advanced endometrial cancer: an interim analysis of a multicentre, open-label, single-arm, phase 2 trial. Lancet Oncol. 20, 711-718 (2019).

367. Ott, P. A. et al. Safety and antitumor activity of pembrolizumab in advanced programmed death ligand 1-positive endometrial cancer: results from the KEYNOTE-028 study. J. Clin. Oncol. 35, 2535-2541 (2017).

368. Varga, A. et al. Pembrolizumab in patients with programmed death ligand 1positive advanced ovarian cancer: analysis of KEYNOTE-028. Gynecol. Oncol. 152, 243-250 (2019).

369. Frenel, J. S. et al. Safety and efficacy of pembrolizumab in advanced, programmed death ligand 1-positive cervical cancer: results from the phase $\mathrm{Ib}$ KEYNOTE-028 trial. J. Clin. Oncol. 35, 4035-4041 (2017).

370. Fader, A. N. et al. Preliminary results of a phase II study: PD-1 blockade in mismatch repair-deficient, recurrent or persistent endometrial cancer. Gynecol. Oncol. 141, 206-207 (2016).

371. Taylor, M. H. et al. Phase IB/II trial of lenvatinib plus pembrolizumab in patients with advanced renal cell carcinoma, endometrial cancer, and other selected advanced solid tumors. J. Clin. Oncol. 38, 1154-1163 (2020).

372. Hao, Z. \& Wang, P. Lenvatinib in management of solid tumors. Oncologist 25 , e302 (2019).

373. Matulonis, U. A. et al. Antitumor activity and safety of pembrolizumab in patients with advanced recurrent ovarian cancer: results from the phase II KEYNOTE-100 study. Ann. Oncol. 30, 1080-1087 (2019).

374. Färkkilä, A. et al. Immunogenomic profiling determines responses to combined PARP and PD-1 inhibition in ovarian cancer. Nat. Commun. 11, 1459 (2020).

375. Naumann, R. W. et al. Safety and efficacy of nivolumab monotherapy in recurrent or metastatic cervical, vaginal, or vulvar carcinoma: results from the phase I/ II CheckMate 358 trial. J. Clin. Oncol. 37, 2825-2834 (2019).

376. Santin, A. D. et al. Phase Il evaluation of nivolumab in the treatment of persistent or recurrent cervical cancer (NCT02257528/NRG-GY002). Gynecol. Oncol. 157, 161-166 (2020).

377. Liu, J. F. et al. 937PDA phase II trial of combination nivolumab and bevacizumab in recurrent ovarian cancer. Ann. Oncol. 29, mdy285-146 (2018).

378. Hamanishi, J. et al. Safety and antitumor activity of anti-PD-1 antibody, nivolumab, in patients with platinum-resistant ovarian cancer. J. Clin. Oncol. 33, 4015-4022 (2015).

379. Fleming, G. F. et al. Clinical activity, safety and biomarker results from a phase la study of atezolizumab (atezo) in advanced/recurrent endometrial cancer (rEC). J. Clin. Oncol. 35, 5585-5585 (2017).

380. Konstantinopoulos, P. A. et al. Phase 2, two-group, two-stage study of avelumab in patients (pts) with microsatellite stable (MSS), microsatelite instable (MSI), and polymerase epsilon (POLE) mutated recurrent/persistent endometrial cancer (EC). J. Clin. Oncol. 37, 5502-5502 (2019).

381. Disis, M. L. et al. Avelumab (MSB0010718C; anti-PD-L1) in patients with recurrent/refractory ovarian cancer from the JAVELIN Solid Tumor phase lb trial: Safety and clinical activity. J. Clin. Oncol. 34, 5533-5533 (2016).

382. Jiao, S. et al. PARP inhibitor upregulates PD-L1 expression and enhances cancerassociated immunosuppression. Clin. Cancer Res. 23, 3711-3720 (2017).

383. Lassen, U. Combining PARP inhibition with PD-1 inhibitors. Lancet Oncol. 20 , 1196-1198 (2019).

384. Westin, S. N. et al. Phase I trial of olaparib (PARP inhibitor) and vistusertib (mTORC1/2 inhibitor) in recurrent endometrial, ovarian and triple negative breast cancer. J. Clin. Oncol. 36, 5504-5504 (2018).

385. Lokich, E. et al. Molecular markers in uterine serous cancer: correlation between endometrial biopsy and hysterectomy specimens. Gynecol. Oncol. Rep. 29, 98-101 (2019).

386. van Kruchten, M. et al. Hormone receptors as a marker of poor survival in epithelial ovarian cancer. Gynecol. Oncol. 138, 634-639 (2015).

387. Zhang, W. et al. Urolithin A suppresses the proliferation of endometrial cancer cells by mediating estrogen receptor-alpha-dependent gene expression. Mol. Nutr. Food Res. 60, 2387-2395 (2016).

388. van Weelden, W. J., Massuger, L., Pijnenborg, J. M. A. \& Romano, A. Anti-estrogen treatment in endometrial cancer: a systematic review. Front. Oncol. 9, 359 (2019).

389. Jerzak, K. J., Duska, L. \& MacKay, H. J. Endocrine therapy in endometrial cancer: an old dog with new tricks. Gynecol. Oncol. 153, 175-183 (2019).

390. Bogliolo, S. et al. The role of fulvestrant in endometrial cancer. Expert Opin. Drug Metab. Toxicol. 13, 537-544 (2017). 
Targeted therapies in gynecological cancers: a comprehensive review of... Wang et al.

391. Blanco, L. Z., Jr. et al. Steroid hormone synthesis by the ovarian stroma surrounding epithelial ovarian tumors: a potential mechanism in ovarian tumorigenesis. Mod. Pathol. 30, 563-576 (2017).

392. Di Leo, A. et al. Buparlisib plus fulvestrant in postmenopausal women with hormone-receptor-positive, HER2-negative, advanced breast cancer progressing on or after mTOR inhibition (BELLE-3): a randomised, double-blind, placebocontrolled, phase 3 trial. Lancet Oncol. 19, 87-100 (2018).

393. Emons, G. et al. Phase II study of fulvestrant $250 \mathrm{mg} / \mathrm{month}$ in patients with recurrent or metastatic endometrial cancer: a study of the Arbeitsgemeinschaft Gynakologische Onkologie. Gynecol. Oncol. 129, 495-499 (2013).

394. Covens, A. L. et al. Phase II study of fulvestrant in recurrent/metastatic endometrial carcinoma: a Gynecologic Oncology Group study. Gynecol. Oncol. 120, 185-188 (2011).

395. Argenta, P. A. et al. A phase II study of fulvestrant in the treatment of multiplyrecurrent epithelial ovarian cancer. Gynecol. Oncol. 113, 205-209 (2009).

396. Gonzalez-Martin, A. et al. Efficacy and safety results from OCTAVIA, a single-arm phase II study evaluating front-line bevacizumab, carboplatin and weeklypaclitaxel for ovarian cancer. Eur. J. Cancer 49, 3831-3838 (2013).

397. Monk, B. J. et al. Randomized phase II evaluation of bevacizumab versus bevacizumab plus fosbretabulin in recurrent ovarian, tubal, or peritoneal carcinoma: an NRG Oncology/Gynecologic Oncology Group Study. J. Clin. Oncol. 34, 2279-2286 (2016).

398. Chambers, S. K. et al. Overexpression of tumor vascular endothelial growth factor A may portend an increased likelihood of progression in a phase II trial of bevacizumab and erlotinib in resistant ovarian cancer. Clin. Cancer Res. 16, 5320-5328 (2010).

399. Verschraegen, C. F. et al. Phase II study of bevacizumab with liposomal doxorubicin for patients with platinum- and taxane-resistant ovarian cancer. Ann. Oncol. 23, 3104-3110 (2012).

400. Musa, F. et al. Phase II study of irinotecan in combination with bevacizumab in recurrent ovarian cancer. Gynecol. Oncol. 144, 279-284 (2017).

401. Tew, W. P. et al. Randomized phase II trial of bevacizumab plus everolimus versus bevacizumab alone for recurrent or persistent ovarian, fallopian tube or peritoneal carcinoma: an NRG oncology/gynecologic oncology group study. Gynecol. Oncol. 151, 257-263 (2018).

402. Tillmanns, T. D. et al. Phase II clinical trial of bevacizumab with albumin-bound paclitaxel in patients with recurrent, platinum-resistant primary epithelial ovarian or primary peritoneal carcinoma. Gynecol. Oncol. 128, 221-228 (2013).

403. Eisenhauer, E. L. et al. A phase II study of gemcitabine, carboplatin and bevacizumab for the treatment of platinum-sensitive recurrent ovarian cancer. Gynecol. Oncol. 134, 262-266 (2014).

404. Aghajanian, C. et al. A phase II study of frontline paclitaxel/carboplatin/bevacizumab, paclitaxel/carboplatin/temsirolimus, or ixabepilone/carboplatin/bevacizumab in advanced/recurrent endometrial cancer. Gynecol. Oncol. 150, 274-281 (2018).

405. Alvarez, E. A. et al. Phase II trial of combination bevacizumab and temsirolimus in the treatment of recurrent or persistent endometrial carcinoma: a Gynecologic Oncology Group study. Gynecol. Oncol. 129, 22-27 (2013).

406. Hagemann, A. R. et al. Phase II study of bevacizumab and pemetrexed for recurrent or persistent epithelial ovarian, fallopian tube or primary peritoneal cancer. Gynecol. Oncol. 131, 535-540 (2013).

407. Richardson, D. L. et al. Paclitaxel with and without pazopanib for persistent or recurrent ovarian cancer: a randomized clinical trial. JAMA Oncol. 4, 196-202 (2018).

408. Pignata, S., Larusso, D. \& Scambia, G. MITO-11: A randomized multicenter phase II trial testing the addition of pazopanib to weekly paclitaxel in platinum resistant or -refractory advanced ovarian cancer (AOC). J. Clin. Oncol. 32, 5503-5503 (2014).

409. Karlan, B. Y. et al. Randomized, double-blind, placebo-controlled phase II study of AMG 386 combined with weekly paclitaxel in patients with recurrent ovarian cancer. J. Clin. Oncol. 30, 362-371 (2012).

410. Vergote, I. et al. A phase II trial of lenvatinib in patients with advanced or recurrent endometrial cancer: angiopoietin-2 as a predictive marker for clinical outcomes. J. Clin. Oncol. 31, 5520-5520 (2013).

411. Audeh, M. W. et al. Oral poly(ADP-ribose) polymerase inhibitor olaparib in patients with BRCA1 or BRCA2 mutations and recurrent ovarian cancer: a proofof-concept trial. Lancet 376, 245-251 (2010).

412. Ledermann, J. A. et al. Overall survival in patients with platinum-sensitive recurrent serous ovarian cancer receiving olaparib maintenance monotherapy: an updated analysis from a randomised, placebo-controlled, double-blind, phase 2 trial. Lancet Oncol. 17, 1579-1589 (2016).

413. Matulonis, U. A. et al. Olaparib monotherapy in patients with advanced relapsed ovarian cancer and a germline BRCA1/2 mutation: a multistudy analysis of response rates and safety. Ann. Oncol. 27, 1013-1019 (2016).
414. Oza, A. M. et al. Olaparib combined with chemotherapy for recurrent platinumsensitive ovarian cancer: a randomised phase 2 trial. Lancet Oncol. 16, 87-97 (2015).

415. Emons, G. et al. Temsirolimus in women with platinum-refractory/resistant ovarian cancer or advanced/recurrent endometrial carcinoma. A phase II study of the AGO-study group (AGO-GYN8). Gynecol. Oncol. 140, 450-456 (2016).

416. Behbakht, K. et al. Phase II trial of the mTOR inhibitor, temsirolimus and evaluation of circulating tumor cells and tumor biomarkers in persistent and recurrent epithelial ovarian and primary peritoneal malignancies: a Gynecologic Oncology Group study. Gynecol. Oncol. 123, 19-26 (2011).

417. Einstein, M. H. et al. Phase II trial of temsirolimus and bevacizumab for initial recurrence of endometrial cancer. J. Clin. Oncol. 30, 5025-5025 (2012).

418. Oza, A. M. et al. Phase II study of temsirolimus in women with recurrent or metastatic endometrial cancer: a trial of the NCIC Clinical Trials Group. J. Clin. Oncol. 29, 3278-3285 (2011).

419. Tinker, A. V. et al. Phase II study of temsirolimus (CCl-779) in women with recurrent, unresectable, locally advanced or metastatic carcinoma of the cervix. A trial of the NCIC Clinical Trials Group (NCIC CTG IND 199). Gynecol. Oncol. 130, 269-274 (2013).

420. Colon-Otero, G. et al. Phase 2 trial of everolimus and letrozole in relapsed estrogen receptor-positive high-grade ovarian cancers. Gynecol. Oncol. 146, 64-68 (2017).

421. Oza, A. M. et al. Randomized phase II trial of ridaforolimus in advanced endometrial carcinoma. J. Clin. Oncol. 33, 3576-3582 (2015).

422. Colombo, N. et al. Ridaforolimus as a single agent in advanced endometrial cancer: results of a single-arm, phase 2 trial. $\mathrm{Br} \mathrm{J}$. Cancer 108, 1021-1026 (2013)

423. Tsoref, D. et al. Phase II study of oral ridaforolimus in women with recurrent or metastatic endometrial cancer. Gynecol. Oncol. 135, 184-189 (2014).

424. Colombo, N. et al. A phase II trial of the mTOR inhibitor AP23573 as a single agent in advanced endometrial cancer. J. Clin. Oncol. 25, 5516-5516 (2007).

425. Lucely, C. et al. A pilot study of nimotuzumab plus single agent chemotherapy as second- or third-line treatment or more in patients with recurrent, persistent or metastatic cervical cancer. Cancer Biol. Ther. 16 684-689 (2015).

426. Santin, A. D. et al. Phase II trial of cetuximab in the treatment of persistent or recurrent squamous or non-squamous cell carcinoma of the cervix: a Gynecologic Oncology Group study. Gynecol. Oncol. 122, 495-500 (2011).

427. Schilder, R. J. et al. Phase II study of gefitinib in patients with relapsed or persistent ovarian or primary peritoneal carcinoma and evaluation of epidermal growth factor receptor mutations and immunohistochemical expression: a Gynecologic Oncology Group Study. Clin. Cancer Res. 11, 5539-5548 (2005).

428. Wagner, U. et al. Gefitinib in combination with tamoxifen in patients with ovarian cancer refractory or resistant to platinum-taxane based therapy-a phase II trial of the AGO Ovarian Cancer Study Group (AGO-OVAR 2.6). Gynecol. Oncol. 105, 132-137 (2007).

429. Hirte, H. et al. A phase II study of erlotinib (OSI-774) given in combination with carboplatin in patients with recurrent epithelial ovarian cancer ( $\mathrm{NCIC} \mathrm{CTG}$ IND.149). Gynecol. Oncol. 118, 308-312 (2010).

430. Blank, S. V. et al. Erlotinib added to carboplatin and paclitaxel as first-line treatment of ovarian cancer: a phase II study based on surgical reassessment. Gynecol. Oncol. 119, 451-456 (2010).

431. Holmberg, L. A., Goff, B. \& Veljovich, D. Unexpected gastrointestinal toxicity from Docetaxel/Carboplatin/Erlotinib followed by maintenance Erlotinib treatment for newly diagnosed stage III/IV ovarian cancer, primary peritoneal, or fallopian tube cancer. Gynecol. Oncol. 121, 426 (2011).

432. Schilder, R. J., Sill, M. W., Yi-Chun, L. \& Robert, M. A phase II trial of erlotinib in recurrent squamous cell carcinoma of the cervix: a Gynecologic Oncology Group Study. Int. J. Gynecol. Cancer 19, 929-933 (2009).

433. Vergote, I. B. et al. A phase 2 randomised discontinuation trial of cabozantinib in patients with ovarian carcinoma. Eur. J. Cancer 83, 229-236 (2017).

434. McNeish, I. A. et al. A randomised, placebo-controlled trial of weekly paclitaxel and saracatinib (AZD0530) in platinum-resistant ovarian, fallopian tube or primary peritoneal cancer. Ann. Oncol. 25, 1988-1995 (2014).

435. Brahmer, J. R. et al. Safety and activity of anti-PD-L1 antibody in patients with advanced cancer. N. Engl. J. Med. 366, 2455-2465 (2012).

436. Liu, J. F. et al. Safety, clinical activity and biomarker assessments of atezolizumab from a Phase I study in advanced/recurrent ovarian and uterine cancers. Gynecol. Oncol. 154, 314-322 (2019). 
Targeted therapies in gynecological cancers: a comprehensive review of...

Wang et al.

34

(c) Open Access This article is licensed under a Creative Commons Attribution 4.0 International License, which permits use, sharing, adaptation, distribution and reproduction in any medium or format, as long as you give appropriate credit to the original author(s) and the source, provide a link to the Creative Commons license, and indicate if changes were made. The images or other third party material in this article are included in the article's Creative Commons license, unless indicated otherwise in a credit line to the material. If material is not included in the article's Creative Commons license and your intended use is not permitted by statutory regulation or exceeds the permitted use, you will need to obtain permission directly from the copyright holder. To view a copy of this license, visit http://creativecommons. org/licenses/by/4.0/.

(c) The Author(s) 2020 